University of South Florida

DIGITAL COMMONS

Digital Commons @ University of

@ UNIVERSITY OF SOUTH FLORIDA

South Florida

February 2020

\title{
Threat-Induced Alterations in Cognition and Associations with Disinhibited Behavior
}

Julia B. McDonald

University of South Florida

Follow this and additional works at: https://digitalcommons.usf.edu/etd

Part of the Clinical Psychology Commons

\section{Scholar Commons Citation}

McDonald, Julia B., "Threat-Induced Alterations in Cognition and Associations with Disinhibited Behavior" (2020). USF Tampa Graduate Theses and Dissertations.

https://digitalcommons.usf.edu/etd/8253

This Thesis is brought to you for free and open access by the USF Graduate Theses and Dissertations at Digital Commons @ University of South Florida. It has been accepted for inclusion in USF Tampa Graduate Theses and Dissertations by an authorized administrator of Digital Commons @ University of South Florida. For more information, please contact digitalcommons@usf.edu. 
Threat-Induced Alterations in Cognition and Associations with Disinhibited Behavior

by

Julia B. McDonald

A thesis submitted in partial fulfillment of the requirements for the degree of Master of Arts Department of Psychology

College of Arts and Sciences

University of South Florida

Major Professor: Edelyn Verona, Ph.D.

Jonathan Rottenberg, Ph.D.

Geoffrey Potts, Ph.D.

Date of Approval:

January 27,2020

Keywords: Disinhibition, Cognitive Control, ERP, Stress, Predictability

Copyright (C) 2020, Julia B. McDonald 


\section{ACKNOWLEDGMENTS}

This study would not have been possible without the support of my incredible family, friends, colleagues, and mentors.

To all of the wonderful members of DARC lab - thank each of your hard work and dedication to the lab. To my brilliant lab mates: Melanie Bozzay, Sean McKinley, Amy Hoffman, Lauren Fournier, and Stephanie Hruza - this project could not have been completed without each of you. To our fierce lab managers: Betty Mendez and Neha Pathak - you were truly the backbone of the lab. And finally, to all of my wonderful research assistants that I have had the privilege of working with over the years - Acree, Alfredo, Ariel, Brianna, Cara, Cristian, Christian, Emilio, Jaime, Joanna, Kashif, Kim, Laura, Lauren, Luz, Malika, Marc, Mary, Manny, Mason, Meghan, Michael, Miranda, Natalie, Nicole, Sass, Sud, and Van. I am excited to see where each of your unique talents lead you!

To my mentor Dr. Edelyn Verona, for providing rigorous training opportunities to contribute to the field while always keeping in mind the broader social implications. Thank you for your patience and support! I am grateful for everything that I have learned from you and I look forward to continuing to pursue our shared interests for years to come.

Special thanks to my committee members Dr. Geoffrey Potts and Dr. Jonathan Rottenberg for your thoughtful suggestions and expertise. And to the National Institute for Mental Health for providing grant funding that made this project possible.

Finally, thank you to my amazing family. To my father for always allowing me to pursue 
my passion. To my brother Jake for always believing in me. And most of all, thank you to my grandmother. Nanny - I owe much of who I am today to you. Thank you for your strength, humor, and encouragement. 


\section{TABLE OF CONTENTS}

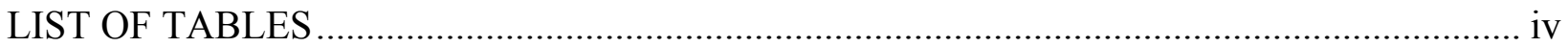

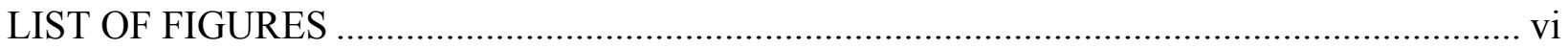

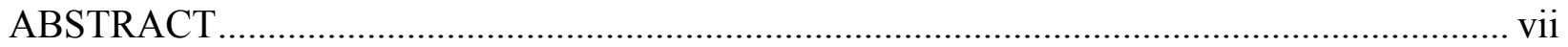

CHAPTER ONE - INTRODUCTION ...................................................................... 1

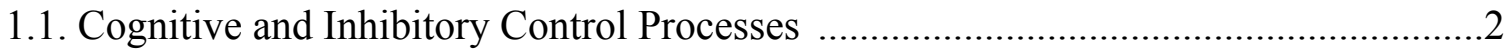

1.2 Threat-Related Disruptions of Cognitive Control..................................................

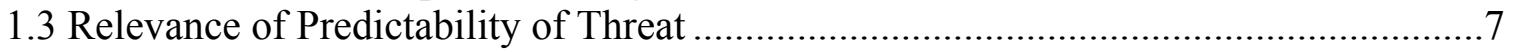

1.4 Threat and Disinhibited Behaviors .................................................................. 10

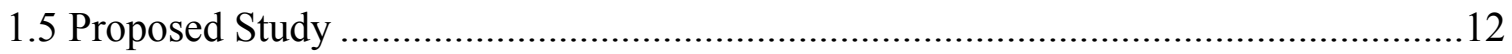

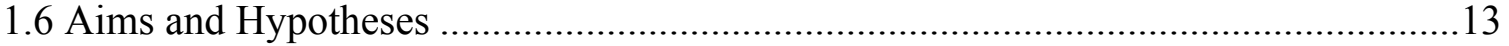

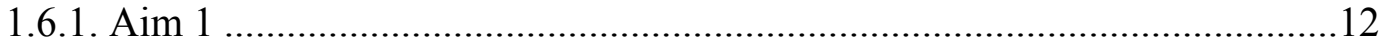

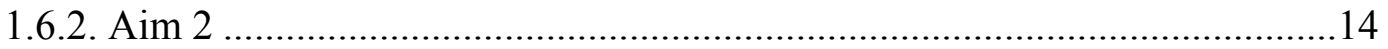

CHAPTER TWO -RESEARCH DESIGN AND METHODS ............................................. 15

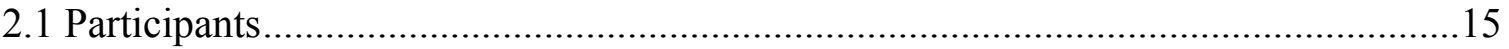

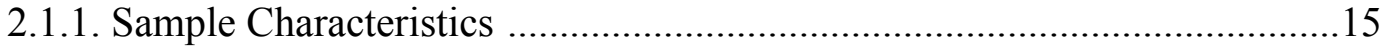

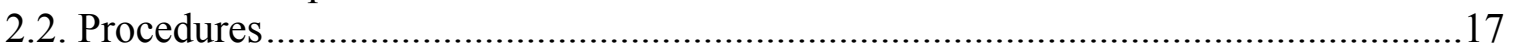

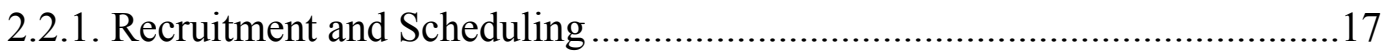

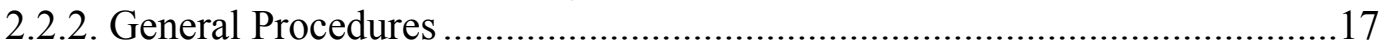

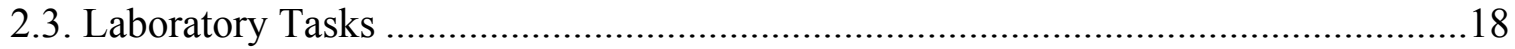

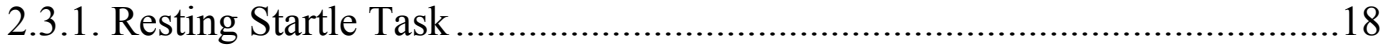

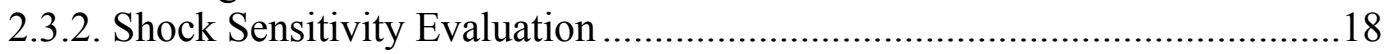

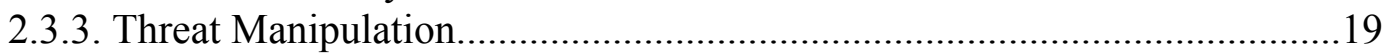

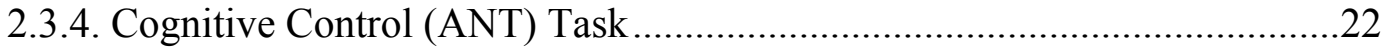

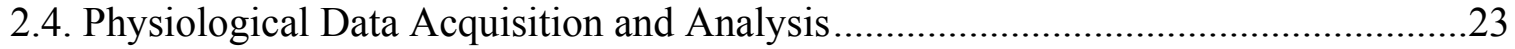

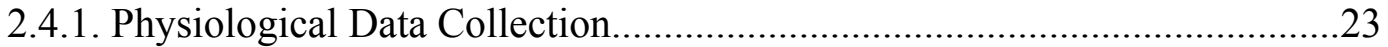

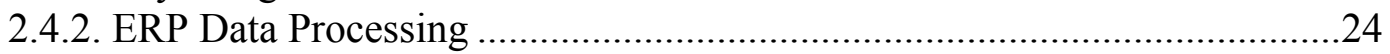

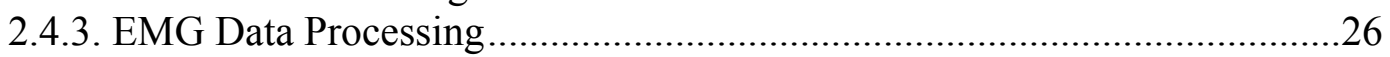

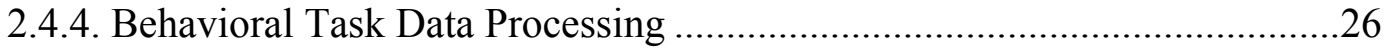

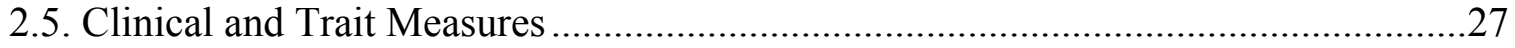

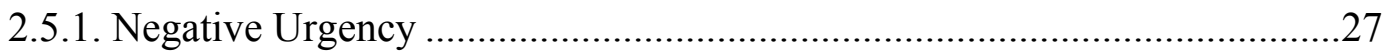

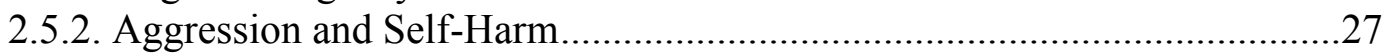

2.5.3. Alcohol and Substance Use Problems .....................................................28

2.6. Possible Covariates and Manipulation Check........................................................29

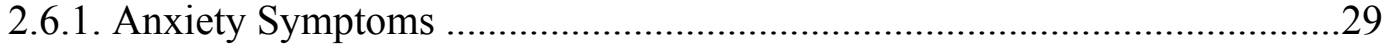

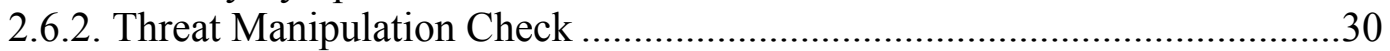




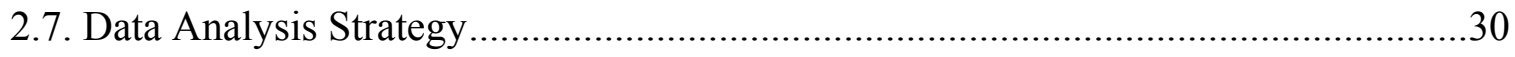

2.7.1. Preliminary Analyses ..............................................................................

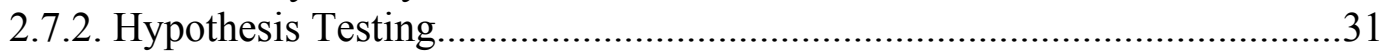

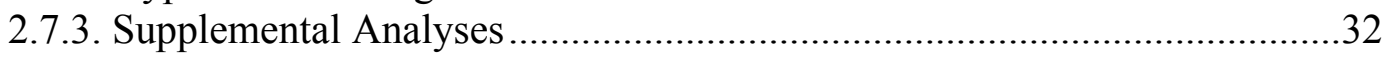

2.7.4. Power Analyses.........................................................................................

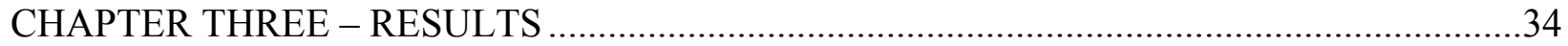

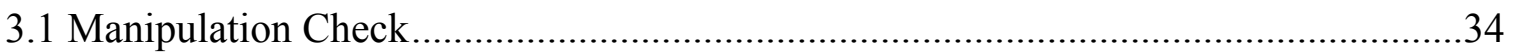

3.2. Aim 1. Impact of Threat on Cognitive Control.........................................................

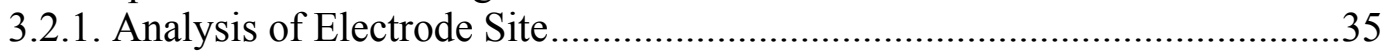

3.2.2. Threat and Cognitive Control: ERP Results ................................................38

3.2.3. Threat and Cognitive Control: Behavioral Results ......................................40

3.3. Aim 2. Associations with Disinhibited Behavior ....................................................42

3.3.1. Disinhibited Behavior Factor Results ........................................................4

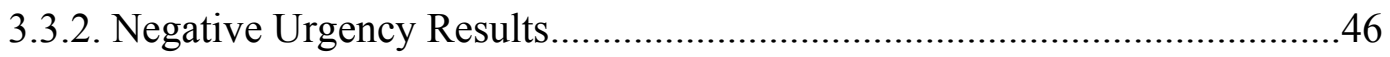

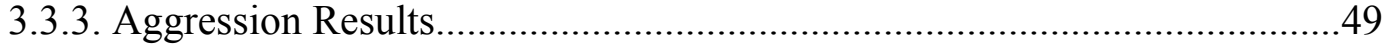

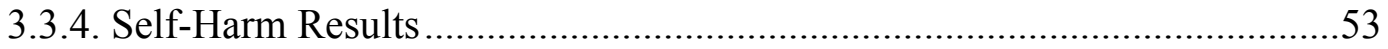

3.3.5. Alcohol Use Disorder Symptoms Results...................................................56

3.3.6. Substance Use Disorder Symptoms Results ..................................................57

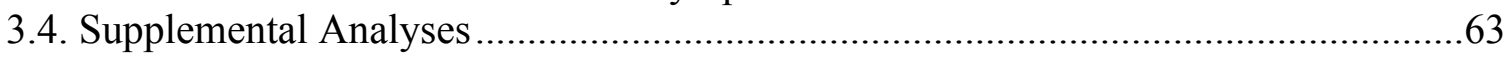

3.4.1. Accounting for Potential Confounds and Covariates...................................63

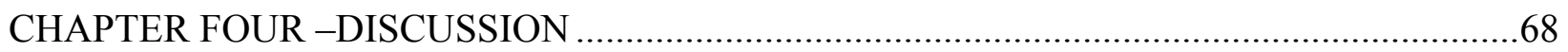

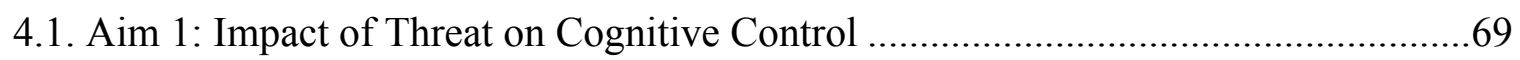

4.2. Aim 2: Associations with Disinhibited Behavior ......................................................71

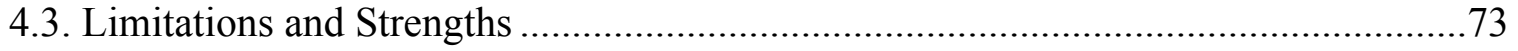

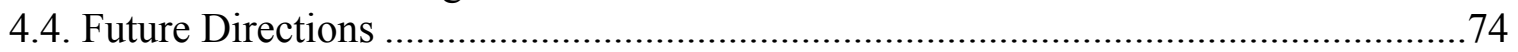

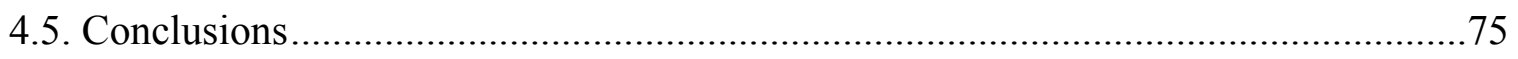

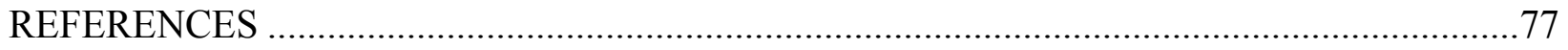

APPENDIX A - RECRUITMENT MATERIALS (PART I - FLYER) .......................................96

APPENDIX B - RECRUITMENT MATERIALS (PART II - ADVERTISEMENT) .................97

APPENDIX C - PRE-SCREENING CONSENT FOR PHONE SCREENING SCRIPT .............98

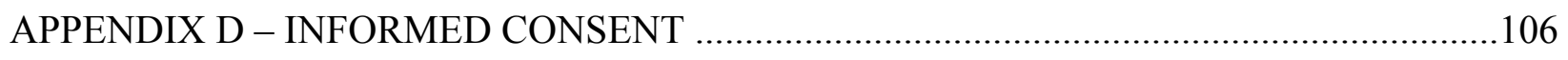

APPENDIX E - PARTICIPANT DEMOGRAPHICS RESPONSE FORM ................................112

APPENDIX F - SHOCK SENSITIVITY EVALUATION FORM ……………………...........115

APPENDIX G - UPPS-P IMPULSIVE BEHAVIOR SCALE …………...............................117

APPENDIX H - LIFETIME HISTORY OF AGGRESSION (LHA) INTERVIEW ...................119 
APPENDIX I - MINI - I. ALCOHOL USE DISORDER (AUD)

APPENDIX J - MINI - J. SUBSTANCE USE DISORDER (SUD) (NON-ALCOHOL) ...........126

APPENDIX K - MOOD AND ANXIETY SYMPTOM QUESTIONNAIRE (MASQ) ............131

APPENDIX L - PENN STATE WORRY QUESTIONNAIRE (PSWQ).....................................133

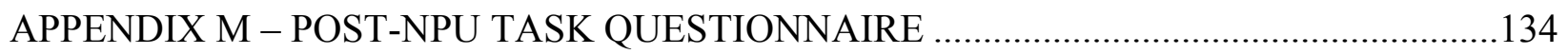

APPENDIX N - POSITIVE AND NEGATIVE AFFECT SCHEDULE (PANAS) …………....136

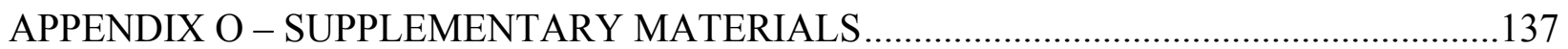




\section{LIST OF TABLES}

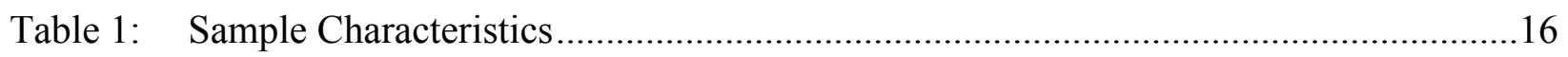

Table 2: $\quad$ Descriptive Summary of ERP and Behavioral Data from NPU-ANT Task...............34

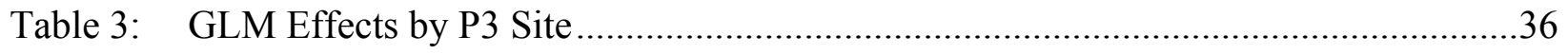

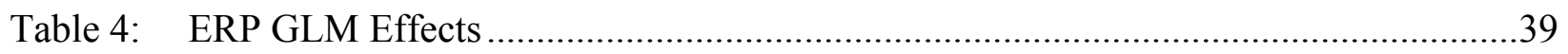

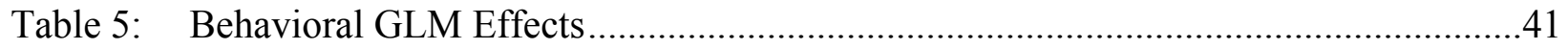

Table 6: Descriptive Statistics and Associations Among Disinhibited Behaviors ...................42

Table 7: Standardized factor loadings of the Disinhibited Behaviors...................................43

Table 8: GLM Effects of ERPs as a function of Disinhibition Composite .............................44

Table 9: GLM Effects of Behavior as a function of Disinhibition Composite .......................45

Table 10: GLM Effects of ERPs as a function of NU.....................................................47

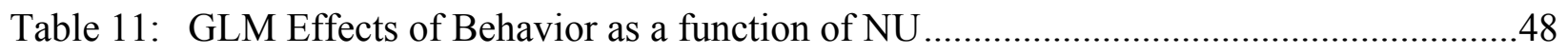

Table 12: GLM Effects of ERPs as a function of Aggressive Behavior..................................50

Table 13: GLM Effects of Behavior as a function of Aggressive behaviors ............................52

Table 14: GLM Effects of ERPs as a function of Self-Harm Behavior...................................53

Table 15: GLM Effects of Behavior as a function of Self-Harm Behavior ..............................54

Table 16: GLM Effects of ERPs as a function of AUD Symptoms.....................................56

Table 17: GLM Effects of Behavior as a function of AUD Symptoms ..................................57

Table 18: GLM Effects of ERPs as a function of SUD Symptoms ......................................58

Table 19: GLM Effects of Behavior as a function of SUD Symptoms ..................................60 


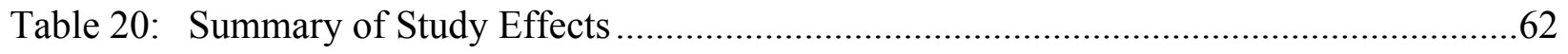

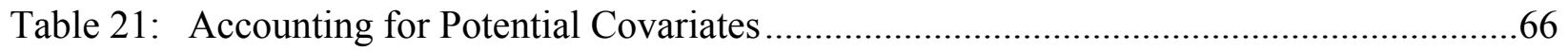




\section{LIST OF FIGURES}

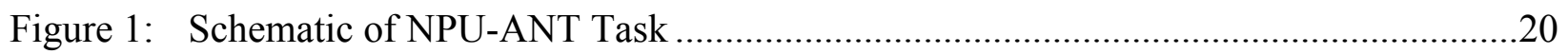

Figure 2: Flanker-Locked ERP Waveforms by Site and Topography …………........................35

Figure 3: Amplitude of P3 Responses to Flanker Congruence by Site ........................................37

Figure 4: ERP Waveforms and Topographies by Condition and Site.........................................38

Figure 5: Accuracy as a Function of Flanker Congruence and Threat Condition........................42

Figure 6: Disinhibition x Overall Threat $x$ Congruence Interaction at Parietal P3 .....................45

Figure 7: Disinhibition x Overall Threat $x$ Congruence Interaction of Accuracy........................46

Figure 8: Negative Urgency x Congruence Interaction at Frontocentral N2 ………..................48

Figure 9: Negative Urgency x Overall Threat Interaction at Frontocentral N2 .........................48

Figure 10: Negative Urgency x Overall Threat Interaction of Accuracy .......................................49

Figure 11: Aggression x Threat Predictability Interaction at Frontocentral N2 ...........................51

Figure 12: Aggression x Overall Threat Interaction at Parietal P3 ……....................................51

Figure 13: Aggression $x$ Overall Threat $x$ Congruence Interaction at Parietal P3 ........................52

Figure 14: Self-Harm x Overall Threat x Congruence Interaction at Frontocentral N2 ...............54

Figure 15: Self-Harm x Congruence Interaction of Reaction Time …………………….............55

Figure 16: SUD x Overall Threat x Congruence Interaction of Parietal P3 …………...................58

Figure 17: SUD x Congruence Interaction of Reaction Time ……………………....................60

Figure 18: SUD x Threat Predictability x Congruence Interaction of Reaction Time..................61

Figure 19: SUD x Overall Threat x Congruence Interaction of Accuracy .....................................61 


\begin{abstract}
When a threat is detected, brain networks associated with threat processing are activated while other processes are deprioritized. While this resource allocation is adaptive, it makes it especially difficult to effortfully direct thoughts, emotions, and behaviors (use cognitive control) during situations of high stress. Further, this threat response is most efficient in response to short-term or predictable stressors ("threats") but loses its efficiency for ambiguous or unpredictable threats. Despite research that suggests that threat induces psychological states associated with breakdown in cognitive control processes, no study has directly examined how predictability of threat impacts neurocognitive indicators of cognitive control processes. Thus, the current study sought to fill this gap by examining whether threat is associated with alterations in cognitive control, and if these threat-related alterations relate to disinhibited and impulsive behaviors (emotion-based rash action, problematic alcohol and drug use, self-harm, and aggressive behavior).

The present study used ERPs to examine threat-related alterations in cognitive control and associations with disinhibited behaviors in a diverse, community sample $(\mathrm{N}=143)$. Participants had their brain activity recorded while completing a flanker task under conditions of predictable, unpredictable, and no threat of shock. Disinhibited behavior was quantified using a combination of self-report measures and semi-structured interviews; and exploratory factor analysis was used to derive a latent disinhibition factor, representing shared variance among the different types of disinhibited behaviors. To determine whether these behaviors relate to ERP
\end{abstract}


indices, each type of disinhibited behavior as well as the shared disinhibition factor were entered separately into regression models.

Findings from the overall sample indicated greater early engagement with flanker stimuli during predictable threat (enhanced frontocentral N2 for predictable vs. unpredictable threat) and reduced overall later-stage processing under conditions of threat, especially predictable threat (blunted parietal P3 for threat vs. no threat and predictable vs. unpredictable threats). This suggests a tradeoff between early vs. later stage attention to flanker during predictable threat blocks. Furthermore, relatively predictable vs. unpredictable threat improved accuracy on the task by reducing accuracy decrements for incongruent trials. Conflict processing (N2 or P3 amplitude to incongruent vs. congruent trials) did not vary as a function of threat condition in the overall sample. Contrary to our predictions, associations with disinhibited behavior revealed a pattern of facilitated processing and improvements in accuracy on more difficult incongruent trials under conditions of stress for those scoring higher vs. lower on reallife disinhibited behaviors.

This research expanded on what is known about threat processing and linked it to high risk behaviors with high societal burden. Previous literature suggests that stress disrupts cognitive control, especially for those prone to engaging in disinhibited behaviors. However, our study suggests a more nuanced relationship, whereby stress influences behavior via reallocation of cognitive processing resources. Depending on the predictability of threat and individual differences in disinhibition, this could actually temporally enhance performance. Our findings provide useful evidence to advance theories of cognitive processing under conditions of threat and disinhibition. 


\section{CHAPTER ONE:}

\section{INTRODUCTION}

The ability to identify and respond to threat is vital for survival. When confronted with a threat (whether it be real or imagined), the brain circuits associated with threat processing are activated, while systems responsible for thinking rationally, inhibiting impulsive behaviors, and self-control are deprioritized (Hermans, Henckens, Joëls, \& Fernández, 2014). While this stress response is adaptive, it can also make it difficult to exercise top-down control to regulate our thoughts and behaviors, often described as cognitive control (also called executive functions; Diamond, 2013; Pessoa, 2009). Furthermore, this threat response system is most efficient in response to short-term, life-threatening, or relatively predictable stressors (or "threats"), but it loses its efficiency for ambiguous or relatively unpredictable threats. That is, being able to identify when it is appropriate to activate the threat response is crucial in order to respond to threats appropriately (Öhman, 2008).

Both experimental and clinical data have demonstrated that breakdowns in cognitive control make individuals more vulnerable to behave in ways that are dangerous to themselves and others (Muraven, Tice, \& Baumeister, 1998; Starcke \& Brand, 2012; Quarantelli, 1954). Not surprisingly, breakdowns in cognitive control have been documented in a variety of problematic behaviors with high societal burden, such as problematic alcohol or drug use (Baler \& Volkow, 2006; Littlefield \& Sher, 2010; Iacono, Carlson, Taylor, Elkins, \& McGue, 1999), violence/ 
aggression (Davidson, Putnam, \& Larson, 2000), and non-suicidal self-injury (NSSI; MullinsSweatt, Lengel, \& Grant, 2012). Research suggests that the reason why these "disinhibited behaviors" often co-aggregate in individuals is in part due to a shared propensity towards either lack of engagement of or breakdowns in cognitive control processes, particularly inhibitory control (also referred to simply as inhibition) (Nigg, 2000).

To date, no study has directly examined the effect of predictability of threat on cognitive control processes and relationships to real-life disinhibited behaviors. First, our research aims to address gaps in the literature by examining how relatively predictable and unpredictable threats impact neurocognitive indicators of cognitive control processes. Second, we examined how threat-induced cognitive control disruptions relate to engagement in disinhibited and impulsive behaviors.

\subsection{Cognitive and Inhibitory Control Processes}

Cognitive control refers broadly to a set of top-down cognitive operations relevant to regulating one's thoughts and behaviors in order to meet one's goals (Botvinick, Braver, Barch, Carter, \& Cohen, 2001; Miyake \& Friedman, 2012). Specific cognitive control functions include inhibitory control [including behavioral inhibition (self-control) and interference control (selective attention and cognitive inhibition)], working memory, and cognitive flexibility (for a

review of cognitive control, see Diamond, 2013). Inhibitory control, a facet of cognitive control, is particularly relevant for disinhibited behaviors, and refers to the ability to control one's attention (i.e., selective attention), behavior (i.e., response inhibition; self-control), thoughts, and/or emotions (i.e., cognitive inhibition) to override a strong internal or external lure and instead do what is appropriate or necessary (Diamond, 2013). These cognitive control processes are activated when it is necessary to focus attention in situations where automatic, intuitive, or 
instinctual processing is ill-advised, ineffective, or insufficient to meet one's goals (Miller \& Cohen, 2001). Cognitive control operations are strongly linked to activity in the prefrontal cortex (PFC) in the brain, particularly the dorsolateral prefrontal cortex (dlPFC) (i.e., the so-called central executive network; Koechlin \& Summerfield, 2007; Menon, 2011).

The ability to control attention is critical to direct processing resources towards stimuli that will promote goal-directed behavior and to suppress irrelevant distractor stimuli (Beck \& Kastner, 2009; Kanske, 2012). This ability to focus attention in the presence of competing stimuli or actions (also known as attentional control or selective/ executive attention) has been studied using conflict processing tasks such as the flanker task (Eriksen \& Eriksen, 1974). The flanker task requires one to exercise inhibitory control of attention in order for individuals to selectively respond to certain stimuli (e.g., center arrow), while ignoring irrelevant or distracting information (e.g., flanking arrows). Trials with distracting information (i.e., incongruent trials) demand relatively more top-down inhibitory control to override inappropriate responses in the presence of conflicting information. Moreover, because incongruent stimuli are more difficult and require more effortful control, trials involving incongruent (vs. congruent) stimuli are associated with prolonged reaction time (RT) and decreased accuracy (Fan, McCandliss, Sommer, Raz, \& Posner, 2002).

The increased effortful control required to process incongruent stimuli has been studied at the level of brain activity using the event-related potential (ERP) methodology, a temporally precise measure of brain activity associated with processing a particular type of stimulus. Specifically, two ERP components are commonly associated with cognitive control processing during the flanker task - the N2 and P3 (Brydges et al., 2012; Folstein \& Van Petten, 2008; Groom \& Cragg, 2015). The N2, a negative-going component that is frontocentrally maximal 
between 200 and $400 \mathrm{~ms}$, is thought to index the deployment of attentional components of cognitive control and/or a marker of response conflict (Heil, Osman, Wiegelmann, Rolke, \& Hennighausen, 2000; Kopp, Rist, Mattler, 1996). In other words, a greater (more negative) N2 amplitude is elicited in response to conflict-laden stimuli that requires top-down control of attention to focus on relevant stimuli (i.e., center arrow) while suppressing attention to irrelevant stimuli (i.e., flanking arrows). In the real world, this might translate to the ability to regulate attention on your work and ignore distractions coming from office noise in order to carry out a task (Diamond, 2013). In contrast, the P3, a positive-going component, is maximal between 300 and $600 \mathrm{~ms}$ and is thought to index later components of cognitive control, such as response evaluation and/ or motor inhibition (Polich, 2007). Of note, two distinct P3 components have been identified: the P3a, which peaks more frontally and reflects automatic attention allocation, and the "traditional" P3b, which peaks more parietally and reflects effortful processing (Polich, 2007). The parietal P3b is typically the component of interest in cognitive control paradigms. For instance, the P3 elicited during the flanker task is thought to index cognitive control of behavior (Groom \& Cragg, 2015).

While the congruence N2 and P3 may index somewhat different aspects of cognitive control (Xie, Ren, Cao, \& Li, 2017), studies that have used simultaneous EEG-fMRI (functional magnetic resonance imaging; a measure of hemodynamic activity within the brain that provides high-resolution spatial information about precise regions of the brain involved in particular cognitive processes) have found that the congruence N2 and P3 overlap in terms of associated neural generators. Specifically, across a range of cognitive control paradigms, activation in the dIPFC, anterior cingulate cortex (ACC), and insula have been associated with the $\mathrm{N} 2$ and $\mathrm{P} 3$, as well as with broader inhibitory control processes (Baumeister et al., 2014; Swick, Ashley, \& 
Turken, 2011; Volpe, Mucci, Bucci, Merlotti, Galderisi, \& Maj, 2007). However, compared to other tasks of inhibitory control, such as the Stroop and go/no-go tasks, less research has examined the neurocognitive correlates of inhibitory control processing during the flanker task (Nee, Wager, \& Jonides, 2007).

\subsection{Threat-Related Disruptions of Cognitive Control}

Research has shown that when confronted with threatening or emotional stimuli, the brain regions associated with cognitive control are deprioritized in favor of evolutionarily older, and less sophisticated response systems (Arnsten, 1998; Liston, McEwen, Casey, 2009). Specifically, when a potential threat is identified, the threat response system (i.e. "fight-or-flight response" or "stress response") is triggered, sending a cascade of signals throughout the body to prepare the organism to respond to the threat (Adolphs, 2013; Sapolsky, 2004). In the brain, threat exposure is associated with the activation of a network of neural circuits known as the salience network (e.g., amygdala, dorsal anterior cingulate (dACC), and anterior insula; Menon, 2015), promoting neuroendocrine reactions that have downstream neuromodulatory effects. Together, these reactions contribute to resource allocation and concomitant downregulation of cognitive control networks (Joëls, \& Baram, 2009). Once the threat subsides, the system should reverse its effects and return to homeostatic balance (Hermans et al., 2014; Schiller \& Delgado, 2010; Öhman, 2008).

Findings regarding cognitive resource allocation under threat are consistent with the "dual competition framework" (for review, see Pessoa, 2009), which posits that because the brain has limited information processing capacity, emotional and cognitive information must compete for processing resources (Potts, Marin, Burton, \& Montague, 2006). From an evolutionary perspective, prioritizing cognitive processes relevant to harm avoidance (e.g., 
spatial navigation and attention to possible threats) at the expense of other cognitive operations (e.g., regulating one's thoughts and emotions, inhibiting impulses) would be adaptive, given that the latter processes do not immediately benefit the organism and require large amounts of cognitive effort (Goldberg \& Grandey, 2007; Muraven, Tice, \& Baumeister, 1998; LeDoux, 2012; Robinson, Vytal, Cornwell, \& Grillon, 2013).

Also consistent with this framework, several experimental tasks have demonstrated that exposure to emotional stimuli induces disruptions in cognitive control (Cohen \& Henik, 2012; Ochsner \& Gross, 2005). For instance, during a version of the emotional Stroop task (McKenna, Frank, Sharma, \& Dinkar, 1995), participants showed poorer performance (e.g., decreased accuracy and prolonged RT) on trials with task-irrelevant negative emotional words (e.g., fear, death, hate) compared to task-irrelevant neutral words (e.g., clock, thumb, potato). Similar effects on behavior have been found in studies that have utilized the Go/NoGo task with emotionally neutral and aversive distractor images (Brown et al., 2012). During these tasks, exposure to emotionally distracting stimuli interfered with the cognitive control of attention and lead to subsequent disruptions in task performance [i.e., slower RT, decreased accuracy]. Much of the evidence that threat exposure impairs cognitive control processing comes from one-time induced threat manipulations such as having to give a public speech or immerse one's hand in ice cold water (cf. Starcke \& Brand, 2012; Starcke, Wiesen, Trotzke, \& Brand, 2016). For example, in a recent study (Jiang \& Rau, 2017), participants that were randomly assigned to a stress condition, in which they were instructed to perform mental arithmetic and public speaking in front of a committee, later showed decreased processing of inhibitory cues (i.e., blunted N2 and P3 difference wave) during a Go/No-Go task, compared to participants in the control condition. Translated outside of the laboratory, emotions have been found to impact behavior in many 
ways, from challenging our ability to focus during lectures, to, at the extremes, limiting our ability to inhibit impulsive or aggressive behavior (Engelmann \& Hare, 2018; Verona \& Bresin, 2015).

Other research has found that the degree to which stress impacted inhibitory control is dependent on individual difference moderators. For example, Dierolf and colleagues (2017) found that participants, in general, showed enhanced inhibitory control (enhanced no-go N2) following a laboratory stress induction; however, those that were highly reactive to the stress task, as evidenced by acute rise in cortisol levels, experienced deficits in the later stages of inhibitory control (i.e., blunted no-go P3). Similarly, a study by Finy and colleagues (2014) found that whether or not an individual engaged in impulsive or risky decision making following a laboratory stress task was dependent personality variables (namely, trait constraint and negative emotionality). Overall, these results confirm the effects of threat on electrophysiological and behavioral indices of cognitive control, but one-time threat induction paradigms are not very informative about ongoing processing of temporally predictive threat stimuli.

\subsection{Relevance of Predictability of Threat}

Many factors influence the nature of the threat response (Sandi, 2013). One aspect of threat that has been found to be of particular importance is the predictability of the threat (Herry et al., 2007; Hsu, 2005). Prior research has confirmed important differences between relatively less than more predictable threat in terms of emotional reactivity and cognitive responses (LeDoux \& Pine, 2016; Öhman, 2008), and responses to unpredictable and predictable threats are evoked by overlapping but distinct neural origins (Adhikari, 2014; Davis, Walker, Miles, \& Grillon, 2010). Specifically, when a threat is more predictable or immediate, it induces a phasic fear response, which is mediated by the amygdala (the brain region associated with processing 
salience and motivationally-relevant stimuli, especially threat), particularly the central nucleus of the amygdala (CeA; Davis, Walker, Miles, \& Grillon, 2010). In contrast, less predictable or contextual threat induces a more sustained, anxious state which is mediated by the bed nucleus of the stria terminalis (BNST), the brain region that forms a bridge between the amygdala and the lateral hypothalamus (Grillon, Baas, Lissek, Smith, \& Milstein, 2004; Torrisi et al,. 2018).

These distinct threat responses have also been mapped onto the Research Domain Criteria (RDoC) criteria as "acute threat" and "potential threat," respectively (Cuthbert \& Insel, 2013). RDoC characterizes acute threat (also termed "fear") as the adaptive, short-term fear response evoked in the presence of a threatening stimulus. In contrast, potential threat (also termed "anxiety") is characterized by the activation of the threat response in the presence of a stressor that may potentially occur but is unpredictable, unlikely in probability, distant, or ambiguous. Studies have examined responses to different aspects of potential threat by manipulating if the threat will occur (probability uncertainty; Hefner \& Curtin, 2011) and when the threat will occur (temporal unpredictability; Davis, 2006; Herry et al., 2007; Grillon, Baas, Lissek, Smith, \& Milstein, 2004). In our study, we manipulated the relative predictability of the onset of an aversive stimulus. Of note, the threat manipulation involved relative predictability. Indeed, in the real world, there are rarely cues that predict the onset of threat with $100 \%$ accuracy.

The ability to have some sense of prediction of the occurrence of a threat is vital because it allows an organism to "turn-on" and "turn-off" the stress response. In contrast, when the occurrence of threat is less predictable, this leads to continued activation of the stress response in the absence of a discernable threat, manifesting in a state of sustained, heightened vigilance; which is a characteristically maladaptive response (Grupe \& Niitschke, 2013; Herry et al., 2007; Sarinopoulos et al., 2009). Indeed, sensitivity to lower predictable threat has been associated 
with pathological functioning such as PTSD, clinical anxiety, and problematic alcohol use in laboratory experiments (Gorka, Lieberman, Phan, \& Shankman, 2016; Grupe \& Nitschke, 2013; Grillon, Pine, Lissek, Rabin, Bonne, \& Vythilingam, 2009). Together these results suggest that stress responses associated with less vs. more predictable threat are linked to behavioral manifestations of maladaptive and disinhibited coping behaviors.

Unfortunately, the majority of research that has examined the impact of characteristics of a threatening stimulus (e.g. predictability, intensity, duration) has focused on affective (rather than cognitive) responses to the threat. For instance, a series of studies has investigated the impact of threatening stimuli (e.g., brief shocks, loud bursts of noise, aversive images) on the startle reflex, a cross-species measure of affective reactivity, elicited by brief, startling acoustic noise probes (i.e. "startle probes") (Blumenthal, Cuthbert, Filion, Hackley, Lipp, \& Boxtel, 2005). The startle eye blink is commonly used to evaluate defensive responding, because the reflex is potentiated (i.e., increased) in emotionally evocative contexts such as under conditions of threat (Bradford, Magruder, Korhumel, \& Curtin, 2014), an effect known as fear potentiated startle or simply startle potentiation. Studies using startle have found that relatively unpredictable threat induces greater defensive responses compared to predictable threat, suggesting that less vs. more predictable threats are especially aversive and/or are associated with the greatest activation of the defensive response system (Schmitz \& Grillon, 2012).

More recently, studies have begun to incorporate electroencephalography (EEG) in the study of threat exposure, although this research still largely focuses on responses to threatening stimuli themselves rather than how threat impacts cognitive processes. For example, a recent study by MacNamara and colleagues (2018) found that, during a threat-of-shock task, processing threatening (vs. non-threatening) stimuli was associated with increased P3 (stimulus-locked to 
the threat cue). This study also found that the P2 amplitude (an ERP associated with early selective attention) was heightened in response to less predictable threat cues relative to more predictable threat cues. Other ERP research has examined threat processing during the anticipation of shocks and unpleasant images and found that the amplitude of the auditory-N1 to noise probes (indexing early attention/ perception) was enhanced while processing relatively unpredictable compared to predictable threats (Nelson \& Hajcak, 2017; Nelson, Hajcak, \&

Shankman, 2015). Together, these findings suggest that the brain allocates additional processing resources towards attending to and categorizing threatening stimuli, especially when the threat is relatively unpredictable. This extra attention to less vs. more predictable threat may lead to reduced allocation of cognitive resources to goal-directed behavior in the context of threat. Unfortunately, this question has not been addressed in previous research.

\subsection{Threat and Disinhibited Behaviors}

Decades of research have shown that disinhibited behaviors result from impairments in inhibitory control and self-regulation (Nigg, 2000). Disinhibited behaviors such as substance use, impulsive self-harm and aggressive behaviors tend to co-occur (Brady \& Sinha, 2005), and all have been associated with reductions in neurocognitive indicators of cognitive control such as N2 (Peterson, 2016) and P3 (Nelson, Patrick, \& Bernat, 2010). Other work also shows that disinhibited behaviors are more likely to occur in the context of negative emotional stimuli. For instance, exposure to negative stimuli or threat, and sustained responses to them, have been associated with more intense aggressive behavior in the lab (Verona \& Kilmer, 2007) and greater behavioral interference (i.e., slower responses in a go/no-go task during negative emotional word blocks) in persons with disinhibited personality traits (e.g., borderline and antisocial personality; Sprague \& Verona, 2010). In ERP research studies, persons with antisocial personality disorder 
(a personality disorder characterized by disinhibited behavioral tendencies) showed enhanced processing of emotional stimuli under inhibitory control conditions (Verona, Sprague, \& Sadeh, 2012). Studies have shown that persons low on inhibitory control and those who exhibit heightened sensitivity to threat show more substance-use related problems (Nelson, Strickland, Krueger, Arbisi, \& Patrick, 2016) and suicide risk (Venables et al., 2015).

These findings linking threat sensitivity to disinhibitory behaviors are interesting because they are consistent with a well-documented literature on how disinhibited behaviors are commonly done in an attempt to regulate overwhelming negative emotions (including threat), or driven by a motivation to cope, albeit maladaptively, with negative emotional states (Tice, Bratslavsky, \& Baumeister, 2001). This theory of emotion regulation is in line with what we know about cognitive and emotional processing during the activation of the stress response, namely the deprioritization of higher-order cognitive systems in favor of survival systems and those that relieve stress/anxiety in the short term (Etkin, Büchel, \& Gross, 2015). This imbalance increases the likelihood of deploying a rapid response with minimal planning (i.e., an impulsive response). Although in some situations impulsive responses may be adaptive for survival, these types of responses may be less advantageous or even harmful to oneself or others in the longer term or at the trait disposition level (Bari \& Robbins, 2013).

Another prominent theory of relevance here is Gray's theory of motivation (Gray, 1987; Gray \& McNaughton, 2000), which proposes two motivational systems that underlie behavior: the behavioral inhibition system (BIS), which corresponds to the motivation to avoid aversive stimuli, and the behavioral activation system (BAS), which corresponds to the motivation to approach appetitive/ rewarding stimuli. Competing approach-versus-withdrawal tendencies are activated in response to different kinds of threat. For instance, it has been 
suggested that in some individuals, the BAS can be activated in the presence of a threat in order motivate behaviors that function to reduce the aversiveness of the stressor or overcome obstacles (Carver, 2004; Verona, Sadeh, \& Curtin, 2009). As such, individuals high in both BIS and BAS tendencies might be motivated to engage in disinhibited behaviors (approach motivation/ BAS) in order to avoid negative affect (avoidance motivation/ BIS) and because of the reinforcing short-term benefits of engaging in such behaviors.

One facet of impulsivity, negative urgency, is reflective of the processes under study here. Negative urgency is defined as the tendency to act rashly under extreme negative emotions or when experiencing distress (Lynam, Smith, Whiteside, \& Cyders, 2006). Not surprisingly, high levels of trait negative urgency have been linked with a variety of disinhibited behaviors such as suicidal behavior (Anestis \& Joiner, 2011), aggression (Settles, Fischer, Cyders, Combs, Gunn, \& Smith, 2012), substance use (Kaiser, Milich, Lynam, \& Charnigo, 2012; Stevens, Blanchard, \& Littlefield, 2018), risky sex (Zapolski, Cyders, \& Smith, 2009), and dysregulated eating behaviors (Anestis, Smith, Fink, \& Joiner, 2008). Despite these studies, however, very little research has fully examined relations between threat-induced cognitive control disruptions and trait negative urgency as well as actual disinhibited behaviors (Kring \& Bachorowski, 1999; Taylor \& Liberzon, 2007; Yancey, Venables, \& Patrick, 2016). Our research aimed to address this gap.

\subsection{Proposed Study}

Our study aimed to 1) investigate the impact of relative predictability of threat on neurocognitive indicators of cognitive control and 2) examine how threat-related disruptions in cognitive control are associated with self-reported disinhibited traits and behaviors (negative urgency, problematic alcohol and drug use, self-harm, and aggressive behavior). This research 
expands what is known about threat processing and links it to high-risk behaviors with high societal burden. This helps us to better understand the factors involved in emotion-induced breakdowns in cognition, which may lead to more effective utilization of therapeutic techniques.

In order to address these research aims, our study utilized a threat of shock paradigm (Schmitz \& Grillon, 2012) combined with a cued cognitive control task (Posner, Snyder, \& Davidson, 1980). This integrated paradigm manipulated the relative predictability of the threat and incorporated an arrow-flanker task with measurement of ERPs. We examined cognitive control processing, with a focus on the N2 and P3 components (stimulus-locked to the flanker), in order to index the effortful inhibition of attention and behavior, respectively (Groom \& Cragg, 2015; Neuhaus, et al., 2010; Patel \& Azzam, 2005). This approach allowed us to measure cognitive control during different shock threat conditions in order to obtain a temporally precise understanding of how relative predictability of threat (more vs. less predictable) impacts cognitive control abilities.

\subsection{Aims and Hypotheses}

\subsubsection{Aim 1. Investigate the Impact of Predictability of Threat on Affective Reactivity} and Cognitive Control. Research suggests that emotions (including emotions induced by threats) are associated with increased physiological arousal and can disrupt the ability to regulate thoughts, inhibit impulses, and effortfully direct attention (Ochsner \& Gross, 2005). Research also suggests that, compared to relatively more predictable threats, less predictable threats can be particularly aversive and as such associated with greater defensive responding, as measured by fear potentiated startle (Schmitz \& Grillon, 2012). In accordance with previous literatures, we expected the unpredictable threat condition, relative to predictable and no-threat conditions, to increase defensive responding (startle potentiation; Baas, Kenemans, Böcker, \& Verbaten, 2002; 
Bennet, Dickmann, \& Larson, 2018; MacNamara \& Barley, 2018) and lead to greater disruptions in neurocognitive indicators of cognitive control (i.e., reduced congruence N2 and P3) across participants. Similarly, we also expected that the unpredictable threat condition would be associated with the poorest performance on behavioral indicators of cognitive control (slower RT and decreased accuracy) in the flanker task (Fan, McCandliss, Sommer, Raz, \& Posner, 2002).

\subsubsection{Aim 2. Link Threat-Induced Disruptions of Cognitive Control to Disinhibited}

Behavior. Disinhibited behaviors have been associated with failures in cognitive control (particularly inhibitory control; Nigg, 2000). These failures of inhibitory control have also been found to be further exacerbated during high-stress situations (Verona \& Kilmer, 2007; Rawls, Jabr, Moody, \& Lamm, 2018), and individual differences in the ability to regulate distress have been associated with real-world manifestations of disinhibited behaviors (Tice, Bratslavsky, \& Baumeister, 2001). Finally, the inability to predict when to appropriately activate the threat response is associated with maladaptive and pathological responses (Grupe \& Niitschke, 2013). Given the results from these studies, we predicted that greater sensitivity to unpredictable threat (startle potentiation) and more unpredictable threat-induced disruptions of cognitive control (e.g., reduced N2/P3) would be related to higher levels of disinhibited traits and behaviors (negative urgency, aggressive behavior, problematic alcohol and drug use, and self-harm). 


\section{CHAPTER TWO:}

\section{RESEARCH DESIGN AND METHODS}

\subsection{Participants}

Data for the present investigation was collected as part of a larger two-part study on aggression in our lab. The focus of the broader study was to investigate the interplay of cognition and emotion and associations with aggression proneness using multiple methodologies including physiology, clinical interviews, self and other reports, and an in-lab aggression manipulation. The purpose of the present study was to examine how different types of threat (relatively predictable, unpredictable) disrupt cognitive control and how these threat-related alterations in cognition related to disinhibited behaviors.

2.1.1. Sample Characteristics. The present study included 151 participants (47\% male, $n$ $=71$ ), actively recruited from the community as part of the larger, grant-funded study in our lab. Inclusion criteria were a) age 18 - 40 years old, b) willing to provide contact information for at least one individual to serve as an informant, for a different aspect of the larger project, and c) able to read English well. Exclusionary criteria consisted of a) specific medical (i.e., epilepsy, traumatic brain injury) and psychiatric (i.e., history of bipolar disorder, schizophrenia, or pervasive developmental disorder) conditions that could contribute to qualitative differences in brain function or cognitive control that could potentially confound our results and b) any hearing or visual impairments. Eight participants were discontinued prior to the completion of the 
Session 1 of the study for the following reasons: because we did not have an EEG net available to fit the participants' heads $(n=4)$, participants voluntarily discontinued because they were uncomfortable with task ( $n=3)$, and because participant had an eye infection $(n=1)$. Therefore, our final sample size for the present study was 143 participants (see demographic information presented in Table 1). Of the 143 participants that completed the task, 138 subjects had useable behavioral data and 114 had useable ERP data. For a full description of excluded ERP and behavioral data, see sections 2.4.3. and 2.4.4, respectively. Our final sample had an even gender split, was fairly racially/ ethnically diverse, mostly employed with low/ middle-income salaries. Finally, our sample was mainly recruited via online ads.

Table 1. Sample Characteristics.

\begin{tabular}{ll}
\hline & Full Sample $(n=143)$ \\
\hline Age $(M(S D))$ & $29.33(6.31)$ \\
Missing $(n(\%))$ & $2(1.4)$ \\
Gender $(n(\%))$ & \\
Male & $67(46.9)$ \\
Female & $72(50.3)$ \\
Transgender & $3(2.1)$ \\
Other & $1(0.7)$ \\
Missing & $0(0.0)$ \\
Race $(n(\%))$ & \\
Caucasian & $80(55.9)$ \\
Black/ African American & $41(28.7)$ \\
Asian & $9(6.3)$ \\
American Indian or Alaskan Native & $4(2.8)$ \\
Other & $8(5.6)$ \\
Missing & $1(0.7)$ \\
Ethnicity $(n(\%$ Hispanic $))$ & $23(16.2)$ \\
Missing & $9(6.3)$ \\
Employment Status $(n(\%))$ & \\
Employed & $113(79.0)$ \\
Unemployed & $20(14.0)$ \\
Homemaker & $7(4.9)$ \\
Other (e.g., Retired) & $2(1.4)$ \\
Missing & $1(0.7)$ \\
Income $(n(\%))$ & \\
$<\$ 15,000$ & $27(18.9)$ \\
\$15-30,000 & $40(28.0)$ \\
\$30-45,000 & $27(18.9)$ \\
\$45-60,000 & $23(16.1)$ \\
\$60-75,000 $\$ 75,000$ & $8(5.6)$ \\
& $15(10.5)$ \\
\hline &
\end{tabular}




\begin{tabular}{ll} 
Table 1. (Continued) & \\
\hline Missing & $3(2.2)$ \\
Recruitment Source $(n(\%))$ & \\
Friend/Relative & $14(9.8)$ \\
Electronic Ads/Flyers & $128(90.2)$ \\
Missing & $1(0.7)$ \\
\hline
\end{tabular}

\subsection{Procedures}

2.2.1. Recruitment and Scheduling. Participants were obtained from the larger study, in which we recruited from the Hillsborough County community through several strategies (see Table 1) including flyers, the local newspaper, and electronic advertisements (Craigslist, Facebook, and employment sites) (see Appendix A and B). Prior to being scheduled, potential participants completed a brief screening over the phone conducted by trained study personnel in order to determine eligibility (see Appendix C). The same eligibility criteria were used for the parent and present study.

2.2.2. General Procedures. This study was approved by the University of South Florida's International Review Board (Pro \# 00027233; see Appendix D). All participants provided written consent prior to their participation.

During Session 1, participants first completed a brief demographics form (see Appendix E), to collect basic information regarding participants' age, gender, race/ethnicity, income and substance use habits (e.g., need for smoke break). Next, participants completed clinical interviews and questionnaires assessing psychopathology and associated behaviors (e.g., depression, substance use disorder symptoms, antisocial traits, suicidal behaviors and NSSI, etc.). All clinical interviews were conducted by trained graduate students, and secondary ratings were completed by trained research assistants for $62.9 \%$ of the interviews, under the supervision of a licensed psychologist. Of note, while the majority of the data examined in the present investigation was collected during Session 1, there were a few self-report measures included in 
this study that were administered during Session 2 (i.e., the UPPS-P Impulsive Behavior Scale, the Mood and Anxiety Symptom Questionnaire, the Pennsylvania State Worry Questionnaire). Because five participants did not complete Session 2 (four no-shows, one voluntarily discontinued immediately upon arrival because of discomfort), our sample size for Aim 2 ( $n=$ $138)$ is smaller than our sample size for aim $1(n=143)$. All physiological data used for ERP/startle analysis for the present investigation were collected during Session 1.

All participants were compensated for their participation. For Session 1, participants had the potential to earn up to $\$ 50$, which included $\$ 35$ for completing the session, $\$ 5$ for transportation reimbursement, and an extra $\$ 10$ bonus for coming to their first scheduled session (without having to reschedule). All participants were debriefed and given a comprehensive list of mental health resources at the end of each session.

\subsection{Laboratory Tasks}

2.3.1. Resting Startle Task. Prior to the main task, we obtained a baseline measure of participants' resting startle. During this resting recording, we instructed participants to passively monitor a fixation cross while we administered nine startle noise probes (i.e., brief (50 ms), loud (102 db) blasts of static white noise used to elicit the startle reflex). No shocks were delivered at this time. This procedure took 2.5 minutes.

2.3.2. Shock Sensitivity Evaluation. Following the resting startle task and before the main procedure, we calibrated the intensity of shocks the participants would receive during the actual threat task to each participants' individual tolerance threshold in order to control for individual differences in shock sensitivity. We accomplished this by having participants rate a series of increasing intensity shocks on a 100-point scale (7mA maximum). Shocks were administered by affixing two shock electrodes ( $8 \mathrm{~mm} \mathrm{Ag-AgCL}$ electrodes) to the tips of their index and ring 
fingers of their non-dominant hand. After each shock was administered, participants were asked to rate how aversive they found the shock on a 100-point scale (i.e., a rating of 0 if they cannot feel the shock at all, a rating of 50 for the first level of shock that they consider to be uncomfortable, and a rating of 100 for the highest level of shock that they can tolerate; see Appendix F). The shock assessment was stopped once the participant rated a shock as the highest level they could tolerate, which they indicated by providing a rating of 100 . Their level of shock rated as 50 (i.e. first level of shock considered uncomfortable) was then used as the shock level for the NPU-ANT Task to induce threat, taking into account individual differences in shock sensitivity. The shock level that participants rated as uncomfortable ranged from .41 to $6.97 \mathrm{~mA}$ $(M=1.82, S D=1.31)$. Also note, there were no significant associations between the shock level rated as uncomfortable and the following dependent variables: $\mathrm{N} 2(r<-.01 p=.97), \mathrm{P} 3(r=-.04$, $p=.70)$, accuracy $(r=.06, p=.51)$, and $\mathrm{RT}(r=.15, p=.08)$. There were also no associations between the shock level rated as uncomfortable and individual differences in disinhibition $(r=$ $.09, p=.28)$, negative urgency $(r=.05, p=.54)$, aggression $(r=.14, p=.10)$, self-harm $(r=-$ $.05, p=.54)$, AUD $(r=-.09, p=.26)$, or SUD symptoms $(r=.10, p=.23)$.

2.3.3. Threat Manipulation. Our paradigm was a modified version of a shock threat paradigm, called the NPU task (Schmitz \& Grillon, 2012), combined with an attentional cueing paradigm, referred to as the Attention Network Task (ANT; Posner et al., 1980) involving an arrow-flanker task (Eriksen \& Eriksen, 1974). The NPU-ANT task combination allowed us to index cognitive control under conditions of predictable threat, unpredictable threat, or no threat (Grillon, 2008). The design of the NPU-ANT task is presented in Figure 1. 


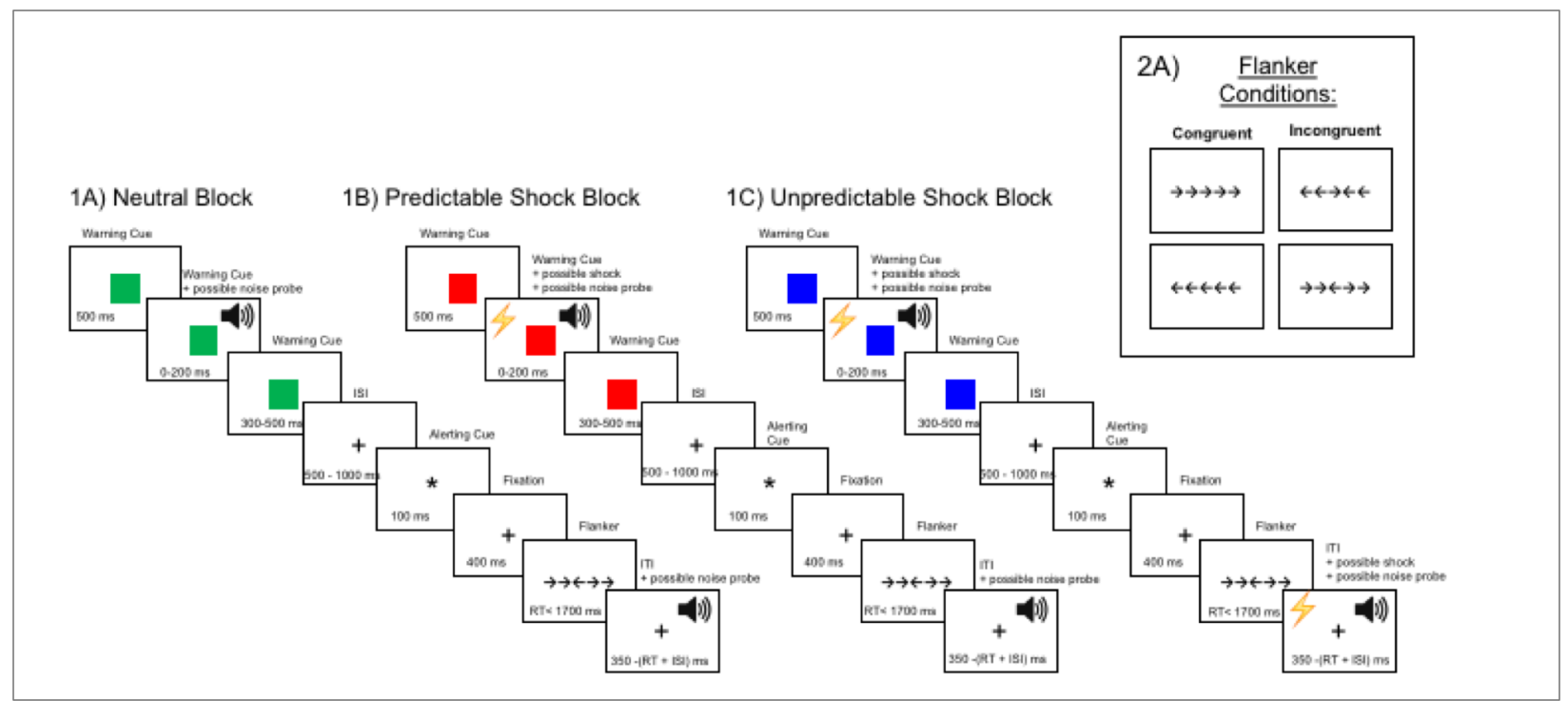

Figure 1. Schematic of NPU-ANT Task.

Each trial of the NPU-task was 5,000-ms. First, the warning cue (square) was presented (1000ms). The color of the warning cue was determined by which type of block the participant was completing (e. g. neutral $(1 \mathrm{~A})=$ green warning cue; predictable $(1 \mathrm{~B})=$ red warning cue; and unpredictable $(I C)=$ blue warning cue). The color of the warning cue indicates the predictability of the shock. During the warning cue for the predictable blocks $(1 B)$ there is a $33.33 \%$ chance of being shocked (50-ms; shock intensity individually calibrated to be "uncomfortable"). During the warning cue for unpredictable blocks (1C) there was a 16.67\% chance of being shocked, whereas the other half of the shocks were administered during inter-trial intervals (thus the unpredictability). Shock administration was pseudo-randomized across trials. Following the warning cue, a jittered inter-stimulus-interval (ISI) occurred (500-1,000-ms). The ISI was followed by the alerting portion of the ANT (alert cue/ no-cue duration $=100-\mathrm{ms}$ ). The alerting period consisted of either an alerting cue (represented by an asterisk, 50\%) or no alerting cue (represented by the continued presentation of the fixation cross, 50\%). Following this cue/ no cue period was a fixed fixation cue period (400-ms), which was then followed by the presentation of the arrow flanker array $(R T<1700-m s)$. Flanker stimuli $(2 \mathrm{~A})$ consisted of an array of arrows that were either congruent (50\%) or incongruent (50\%). Finally, following the arrow-flanker, there was a pseudo-random inter-trial interval (ITI; 350 - (RT + ISI) that consisted of a fixationcross prior to the start of the next trial. During the ITI for unpredictable blocks (1C) there was a $16.67 \%$ chance of being shocked. Of note, during each of the 9 blocks, 9 startle noise probes (50-ms, 102-db white noise) were presented pseudo-randomly to elicit the startle response, with $50 \%$ occurring during the warning cue and 50\% occurring during the ITI. Participants were instructed to focus on the flanker task, despite the shocks and startle noise probes.

A typical NPU threat task consists of 3 conditions: a neutral $(\mathrm{N})$, predictable $(\mathrm{P})$, or unpredictable (U) condition. During the $\mathrm{N}$ condition, participants are safe from the aversive stimulus (shock). In the P condition, participants are shocked, but shocks are signaled by a threat warning cue. By contrast, during the $\mathrm{U}$ condition, participants are shocked, but shocks are not 
signaled. While there are broad range of "stress" manipulations (cf. Starcke \& Brand, 2012) for the present study, we chose to focus on the threat of shock paradigm (Schmitz \& Grillon, 2012) as it is a well-validated, translational, and robust threat manipulation (Davis, Walker, Miles, \& Grillon, 2010; Schmitz \& Grillon, 2012; Walker \& Davis, 2008). Prior to the start of each block, an instructions screen appeared informing participants of the type of block they were going to be completing next (i.e. N, P, or U block; see Figures 1A - 1C). There were nine blocks total (3 of each type). Each block contained 30 trials, for a total of 270 trials. The order of the blocks was counterbalanced using a Latin square technique, between participants (i.e. order 1:

PUNUNPNPU; order 2: UPNPNUNUP) ${ }^{1}$. In between each block, the participant could take a short break; every three blocks, the experimenter went into the participant room and checked impedances, adjusting electrodes to lower the impedances if necessary. Each block took 2.5 minutes, and the whole NPU-ANT task took 30 minutes.

During predictable shock blocks (see Figure 1.1B), participants were shocked only during the appearance of a red square on the screen (referred to as the warning cue). Although not all predictable threat trials involved a shock administration ( $33 \%$ of these involved shock), when the shock was administered, it only did so while the warning cue was on the screen. Despite predictable threat cues only being predictive of when a shock would occur $33.33 \%$ of the time, this cue was still quite informative about when the threat would occur, especially compared to the types of threat cues that exist in the real world. During unpredictable shock blocks (see Figure 1.1C), a colored square also appeared at the beginning of the block; however, this time the square was blue. During unpredictable shock blocks, participants were told that they could be shocked at any point during the trial. They received shocks sometimes when the blue square was

\footnotetext{
${ }^{1}$ The first 25 participants were all ran as order 1 . Then, participant orders were pseudo-randomly assigned to orders such that 75 people were ran for order 1, and 69 people were ran for order 2.
} 
on the screen, and other times during inter-trial intervals (ITIs, which were not of fixed duration), following the flanker task. Lastly, we included a no-threat condition (referred to as neutral blocks; see Figure 1.1A) when participants were not shocked at any point during these blocks. Participants still saw a square prior to the ANT task during the neutral block; however, this time the square was green, and it indicated safety from the shock.

2.3.4. Cognitive Control (ANT) task. The ANT task we used consisted of the attentional alerting and attentional control portions. ${ }^{2}$ The attentional alerting portion of the ANT task manipulates the presence or absence of a cue (represented by the presence or absence of an asterisk) as a measure of attentional alerting. On some trials, participants saw a very brief (100ms) presentation of an asterisk (alerting cue), which indicates the imminent appearance of the flanker. This alerting cue is not analyzed as part of this study, which focuses on cognitive control and not attentional alerting. Following the cue/no cue period, the second portion of the task assesses attentional or cognitive control, using the arrow flanker task (Eriksen \& Eriksen, 1974). Participants are told to indicate the direction of a central arrow in an array (see Figure 1.2A). On congruent trials, all arrows are pointed the same way (e.g. $\rightarrow \rightarrow \rightarrow \rightarrow \rightarrow$ or $\leftarrow \leftarrow \leftarrow \leftarrow \leftarrow$ ). During incongruent trials, the central arrow is pointing in the opposite direction as the flanking arrows on either side (e.g. $\rightarrow \longrightarrow \leftarrow \rightarrow \longrightarrow$ or $\longleftarrow \leftarrow \rightarrow \leftarrow \leftarrow$ ). The order of the congruent and incongruent trials was pseudo-randomized and equiprobable. Performance on congruent trials is automatic, only requiring basic perceptual and motor abilities. In contrast, performance on the incongruent trials is more difficult, requiring the participant to select an appropriate response based on perceptually similar stimuli, while ignoring distracting stimuli (i.e. ignoring the flanking arrows). Thus, it is necessary to exert more cognitive control on incongruent (vs. congruent) trials. This

\footnotetext{
${ }^{2}$ Of note, the standard version of the ANT task includes an orienting portion that was not included in this variant.
} 
results in incongruent (vs. congruent) trials having poorer task performance (e.g., increased RT and decreased accuracy; Fan, McCandliss, Sommer, Raz, \& Posner, 2002).

\subsection{Physiological Data Acquisition and Analysis}

2.4.1. Physiological data collection. Physiological data were collected using Electrical Geodesics system hydrocel 64-channel sensor nets and amplifiers (EGI, Eugene, OR). EEG sensor nets were soaked in a warm potassium chloride solution for up to ten minutes prior to use. High-density hydrocel geodesic sensor nets are associated with high-impedance amplifiers and are designed to accept impedances as high as $100 \mathrm{k} \Omega$ (Ferre, Luu, Russel, Tucker, 2001). EEG impedances were kept below $50 \mathrm{k} \Omega$. During the data collection both the EMG and EEG data were continuously recorded at a sampling rate of $1000 \mathrm{~Hz}$ and referenced to the vertex during online recording, later to be re-referenced during offline analysis. Analog signals were amplified online using Net Amps 400 amplifiers. EEG data was filtered at $0.1-0.100 \mathrm{~Hz}$.

Startle electromyogram (EMG) data was collected using the Physio16 input box, which integrates with EGI's Geodesic EEG system. Startle activity was recorded from two 4mm AgAgCL sensor placed on the orbicularis oculi muscle underneath the participants' left eye, and according to published guidelines (cf. Blumenthal, 2005). Psychometric studies have shown that the startle eye blink is a reliable measure of affect during the NPU task (Kaye, Bradford, \& Curtin, 2016). Also, compared to self-report measures of affect, startle potentiation is resistant to demand characteristics, and has clear connections to neurobiological substrates, making it the ideal dependent measure of negative affect in threat manipulation studies (Kaye et al., 2016).

All physiological data was continuously recorded using Net Station 5.3.0.1 on an iMac running OS X 10.11.6. Stimulus presentation and behavioral response collection utilized version 2.0.10.356 E-Prime software (PST Inc., Pittsburgh, PA) running Windows 7. Audio stimuli 
(startle noise probes) were presented using Sennheiser HD 202 II Professional headphones connected to a Creative SB ZxR audio card. The startle probe was a 50-ms, 102 decibel (dB) white noise probe (instantaneous rise-time), calibrated weekly by a sound pressure meter. Visual stimuli were presented on a Dell 22-inch LCD monitor in the participant room. Behavioral responses were collected with a 4-key keypad that interfaces to E-Prime (although only 2 responses were required for the task).

2.4.2. ERP Data Processing. Offline data processing was completed in Net Station 5.4 .2 (Eugene, OR) for psychophysiological data reduction. EEG data was then band-pass filtered at .3 to $30 \mathrm{~Hz}$ (Passband Gain: 99.88\% [-0.01 dB], Stopband Gain: 1.0\% [-40.0dB], Rolloff: $2.0 \mathrm{~Hz}$ ), and segmented into 1000-ms epochs by condition (total of 6 conditions; 2 congruent conditions: congruent and incongruent, 3 threat conditions: N, P, U). Epochs were segmented to the onset of the flanker stimulus from 200-ms before and 800-ms after flanker stimulus onset. Only error-free trials were used in analyses. Artifacts were automatically detected and manually verified for exclusion from additional analysis (bad channel $>200 \mu \mathrm{V}$, eye blinks $>140 \mu \mathrm{V}$ and eye movement $>55 \mu \mathrm{V})$. For every channel, $20 \%$ or greater bad segments was used as the criteria for marking that channel bad. Bad channels (fluctuations over $200 \mu \mathrm{V}$ ) were then be spherical spline interpolated from nearby electrodes. For every segment, greater than 10 bad channels was used as a criterion for marking the whole segment bad. Following data processing, an average of $73 \%$ of trials were retained for $\mathrm{N}$ and $\mathrm{P}$ threat conditions, and $71 \%$ of trials were retained for $\mathrm{U}$ threat condition (Range 0-100\%, 0-90 trials). Next, participants with less than $25 \%$ usable segments in any condition (out of 45 total possible trials per each of the 6 conditions) were excluded from the ERP analyses to ensure we had at least the minimum number of trials needed for a statistically stable (e.g., internally consistent) ERP. Following these procedures, a total of 114 subjects 
(80.9\% of valid EEG files) were retained in ERP analyses ${ }^{3}$. Of note, we excluded a somewhat higher rate of participants compared to previous ERP studies using ANT/ flanker tasks without concurrent shock manipulation; these exclusions occurred due to artifacts from excessive muscle activity $(\mathrm{n}=27)$. However, these exclusions were expected given that participants moved more during our task from receiving shock stimuli. Data were baseline adjusted using a 200-ms prestimulus period and then re-referenced from vertex recording to an average reference of all 64 channels.

In order to characterize the internal reliability of the ERP components within the present sample, we computed dependability estimates using the ERP Reliability Analysis (ERA) Toolbox v 0.4.8 (Clayson \& Miller, 2017), which uses formulas based on generalizability theory (Baldwin, Larson, \& Clayson, 2015). Results suggested that the internal reliability of our ERP components for each condition was good, with dependability estimates ranging from .78 to .84 for the $\mathrm{N} 2$ and .83 to .89 for the $\mathrm{P} 3$.

All processed, artifact-free segments were then averaged together for each condition (threat: N, P, U; congruence: incongruent, congruent) to produce an individual average waveform per participant. Additionally, all conditions were then averaged across all participants to produce a single grand average. This grand average was used for visual inspection of the waveform and scalp topography to aid in the selection of N2/P3 component time-windows and examine where on the scalp effects were maximal, respectively. Grand average ERP waveforms were examined at frontocentral, central, and parietal sites (see Figure 2) based on where effects

\footnotetext{
${ }^{3}$ Subjects excluded from ERP analyses did not significantly differ from included subjects on most demographic variables (e.g., age, gender, race, occupation, education, income), except that a significantly greater percentage of excluded subjects identified as Hispanic $(40.00 \%)$, compared to those included $(16.28 \%), t(132)=11.57, p=.00$. Also, subjects excluded from ERP analyses did not significantly differ on most primary study variables (e.g., impulsivity, aggressive behavior, alcohol and substance use symptoms), with the exception that those excluded from the ERP analyses reported significantly lower rates of self-harm $(M=.24, S D=.83)$ compared to those included $(M$ $=.96, S D=1.70), t(141)=16.92, p=.00$.
} 
appeared maximal and based on previous literature (Brydges et al., 2012; Clayson \& Larson, 2013; Groom \& Cragg, 2015; Rietdijk, Franken, \& Thurik, 2014).

For both the N2 and P3 (separately), the adaptive mean was computed, which identified individual windows for each participant to account for subtle differences in waveform morphology across participants. The N2 component was defined as the minimum mean-peak amplitude (average amplitude across +/- 15-ms around the peak) detected between 280 to 350ms post-flanker stimuli at frontocentral sites (see Figure 2 for electrode montage; Nieuwenhuis, Yeung, Van Den Wildenberg, \& Ridderinkhof, 2003; Rietdijk, Franken, \& Thurik, 2014). The P3 component was defined as the maximum mean-peak amplitude (average amplitude across $+/$ 25-ms around the peak) detected between 300 to 500-ms post-flanker stimuli at the parietal site (see Figure 2 for electrode montage; Rietdijk, Franken, \& Thurik, 2014). The adaptive mean for the N2 and P3 for each subject was then exported for statistical analysis.

2.4.3. EMG Data Processing. Startle EMG data was processed offline in Matlab using EEGLAB (Delorme \& Makeig, 2004) and according to published guidelines (cf. Blumenthal, 2005). Data were filtered to reduce noise using a 28 - $500 \mathrm{~Hz}$ filter, signal rectified, and smoothed with a $30 \mathrm{~Hz}$ low-pass filter, then segmented by condition (total of 6 conditions: Cue Probe and ITI Probe for each of the 3 threat conditions: N, P, U). Epochs were segmented 100ms before and 250-ms after startle probe onset. Visual inspection of segmented startle data revealed a high degree of noise contamination and an indiscernible startle response. Given that we were unable to detect a valid startle response, we did not analyze the startle data.

2.4.4. Behavioral Task Data Processing. We analyzed behavioral indicators of cognitive control, including accuracy (percentage correct) and RT, as additional analyses of our primary aims. Mean RTs and accuracy were calculated separately for each experimental condition (i.e., 
congruent, incongruent and N, P, U threat conditions). Five participants were excluded due to corrupted $(n=1)$, incomplete $(n=4)$, or missing data files $(n=1)$. Therefore, of the 143 participants that completed the task, 138 had useable behavioral data.

\subsection{Clinical and Trait Measures}

2.5.1. Negative urgency. All participants completed the UPPS-P Impulsive Behavior Scale (Lynam, Smith, Whiteside, \& Cyders, 2006; see Appendix G) during Session 2. The UPPS-P is a 59-item self-report questionnaire measuring dispositional impulsivity. Each item is rated based on a 4-point scale (ranging from 1 = "disagree strongly" to 4 = "agree strongly"), with higher scores indicating greater impulsivity. The subscales within the UPPS-P include: 1) negative urgency $(\alpha=.93)$ : tendency to act rashly under extreme negative emotions, 2) positive urgency $(\alpha=.95)$ : tendency to act rashly under extreme positive emotions, 3 ) lack of premeditation $(\alpha=.86)$ : tendency to act without thinking, 4$)$ lack of perseverance $(\alpha=.85)$ : inability to remain focused on a task, and 5) sensation seeking $(\alpha=.84)$ : tendency to seek out novel and thrilling experiences. These subscales can be summed together to create a total impulsivity score (total score $\alpha=.94$ ). For the present study, we were most interested in the negative urgency subscale of the UPPS-P because it allowed us to index individual proclivities towards impulsive behaviors in response to experiencing negative emotions (or threat).

\subsubsection{Aggression and Self-Harm. During Session 1, we administered the Lifetime}

History of Aggression (LHA) Interview (Brown et al., 1982; see Appendix H), a semi-structured interview of aggressive history. The LHA was used in order to assess the association between threat-induced disruptions in cognitive control and self-reported aggressive behavior in participants' lives (including LHA-self-directed facet which includes suicidal and non-suicidal self-injury). Participants were rated on the frequency of 11 types of aggressive and antisocial 
behavior occurring since the age of 13. Ratings were based on a 5-point scale (ranging from $0=$ "no events" to 5 = "so many events they cannot be counted"). Subscales of the LHA used in analyses were 1) Aggression, which includes items measuring temper tantrums, physical fights, and assaults on property, and 2) Self-Directed Aggression, which includes two items measuring non-suicidal self-injurious behavior and suicide attempts. For the present study, we used sum scores of the LHA-Aggression scale $(\alpha=.69$, individual item correlations ranged from $r=.15$ .46) to estimate engagement in aggressive behaviors, and the sum scores of the LHA-SelfDirected scale $(\alpha=.26)$ were used as a measure of self-harm (both suicidal and non-suicidal). Inter-rater agreement was excellent for both the Aggression $(\mathrm{ICC}=.98)$ and Self-Directed Aggression $(\mathrm{ICC}=.94)$ subscales of the LHA $(\mathrm{Koo} \& \mathrm{Li}, 2016)$.

\subsubsection{Alcohol and Substance Use Problems. Symptoms of self-reported alcohol (see} Appendix I) and substance use disorder (see Appendix J) were assessed during Session 1 using the Alcohol and Substance Use Disorder modules of the MINI-International Neuropsychiatric Interview (MINI - 7.0 Sheehan, 2014). These were used to examine the associations between threat, cognition, and substance use (Kaiser, Milich, Lynam, \& Charnigo, 2012; Stevens, Blanchard, \& Littlefield, 2018). The MINI is a semi-structured interview for the Diagnostic and Statistical Manual of Mental Disorders - 5th Edition (DSM-5; American Psychiatric Association, 2013) and demonstrates good validity and reliability in assessing Alcohol Use Disorder (AUD) and Substance Use Disorders (SUD; Sheehan, 2014). Given previous research that has linked problems with alcohol and drug use to heightened reactivity under conditions of uncertain threat (Gorka, Nelson, \& Shankman, 2013; Gorka, Lieberman, Phan, \& Shankman, 2016), we were particularly interested in how drug and alcohol use disorder symptoms (not just use) relate to threat-induced disruptions in cognitive control. As such, we used symptom counts (number of 
symptoms rated as threshold vs. non-threshold) corresponding to the maximum number of lifetime (last year or past) DSM-5 criteria (11 symptoms) met for each condition (i.e., both AUD and SUD) at any time in the participants' life. Both AUD $($ ICC $=.94)$ and SUD $($ ICC $=.97)$ modules of the MINI had excellent inter-rater agreement (Koo \& Li, 2016).

\subsection{Possible Covariates and Manipulation Check}

2.6.1. Anxiety Symptoms. Previous literature has shown that symptoms of anxiety are associated with exaggerated psychophysiological responses to threat cues (Davis et al., 2010). Therefore, in order to adjust for impact of individual differences in anxiety in our analyses, we administered two measures of anxiety (indexing both physiological and cognitive symptoms) during Session 2. First, the modified version of the Mood and Anxiety Symptom Questionnaire (MASQ; see Appendix K) was administered (cf. Watson, Clark, Weber, Assenheimer, Strauss, \& McCormick, 1995). The measure consisted of 39-items from the anxious arousal and anhedonic depression subscales of the MASQ. For the present study, we were only interested in utilizing the anxious arousal subscale of the MASQ, in order to index physiological symptoms of anxiety. Participants were instructed to rate the extent to which they experienced a given symptom (e.g., "startled easily", "had shortness of breath") over the past two weeks on a 5-point Likert scale (1 $=$ "not at all" to 5 = "extremely"). Second, the Penn State Worry Questionnaire (PSWQ; Meyer, Miller, Metzger, \& Borkovec, 1990; see Appendix L) was administered to index cognitive worry symptoms of anxiety. The PSWQ consists of 16-items on a 5-point Likert scale (1= "not at all typical of $m e$ " to $5=$ "very typical of me"). Total scores of the PSWQ have shown to relate to symptoms of worry in community samples (Gillis, Haaga, \& Ford, 1995). The total score for the MASQ anxious arousal subscale $(\alpha=.86)$ and total score for the PSWQ $(\alpha=.94)$ were used as 
covariates in analyses of Aim 2 to examine the specificity of the link between cognitive disruptions and disinhibited behaviors versus psychopathology more broadly.

2.6.2. Threat Manipulation Check. The adequacy of the threat manipulation was assessed using a questionnaire (adapted from Bradford, Magruder, Korhumel, \& Curtin, 2014; see Appendix M) administered immediately following the experiment and measured participants' retrospective recall of their anxiety, fear, attention, and motivation during the experiment and during each threat condition (each item was assessed using a 5-point Likert scale, ranging from 0 to 4). In addition to the Post NPU-Task, a post-study interview was used as an additional gauge of the participants' experience of the task (e.g., "how was the task overall?", "how did you feel during the red block", "was there anything strange about the task," etc.). This interview was conducted to screen for any possible validity concerns (e.g., misunderstanding task, not hearing any noises, not feeling the shock, etc.). Participants with validity concerns were flagged and screened to ensure their data were valid.

\subsection{Data Analysis Strategy}

2.7.1. Preliminary Analyses. Prior to hypothesis testing, all data were screened for violations to the assumptions of normality by inspecting skewness and kurtosis for each variable. Data were also inspected for outliers. Any non-normal dependent variables were log transformed when appropriate in order to meet the underlying assumptions of our models (Cohen, Cohen, West, \& Aiken, 2002; Keene, 1995). We log transformed the Self-Directed Aggression (selfharm) subscale of the LHA (skewness $=2.00$, kurtosis $=3.41$ ). Log transformed values are presented in Table 2. We also examined the associations of our disinhibited behavior variables using Pearson's bivariate correlations (see Table 2). Disinhibited behaviors were first combined into composite variable, if deemed to assess a similar construct. Specifically, we used 
Exploratory Factor Analysis (EFA) to estimate a general disinhibition factor and extract the resulting factor scores to use in subsequent analyses to examine the interaction of shared disinhibition, threat, and flanker congruence processing.

2.7.2. Hypothesis Testing. For Aim 1, ERP data were analyzed using a series of repeated measures GLMs, in order to examine the separate and interactive effects of threat context and flanker congruence on cognitive control. Each dependent measure (N2, P3, RT, accuracy) was analyzed in a separate repeated measures GLM. Threat condition effects were parsed into two orthogonal a-priori planned contrasts: 1) overall threat effect: predictable threat and unpredictable threat vs. no threat (i.e. P/U vs. N) and 2) (un)predictability: relatively unpredictable vs. predictable threat (i.e., P vs. U). Only the within subject contrasts were interpreted, not the multivariate effects. The decision to use planned contrasts helped to control the false-positive rate and was also theoretically meaningful because it allowed us to parse between the overall threat effect as well as the effect of relative (un)predictability on cognitive control. Significant 2-way interactions (threat $\mathrm{x}$ congruence) were followed up by simple effects testing within one of the factors (e.g. threat, congruence) (Aiken \& West, 1991). This allowed us to examine the effect of threat at each level of congruence, and congruence at each level of threat, respectively. Again, we expected that 1) overall threat (vs. no threat) and 2) unpredictable (vs. predictable) threat would be associated with slower RT, poorer accuracy, and decreased N2 and P3 processing overall (i.e., main effects of threat on ERP). We also predicted that cognitive processes related to conflict detection (i.e., differential processing of incongruent versus congruent flanker stimuli), would be disrupted under conditions of threat, especially unpredictable threat (i.e. Threat $\mathrm{x}$ Congruence interaction). Effect sizes were reported for all 
GLM results using generalized Eta-Squared $\left(\eta^{2}\right.$; Cohen, 1988): $\eta^{2}$ of 0.01 is considered a small effect size, 0.06 is considered a medium effect, and 0.14 or larger indexes a large effect size.

For Aim 2, we examined the associations between threat-related disruptions of cognitive control and disinhibited traits and behaviors. We assessed this by adding measures of disinhibited traits or behaviors, first as a composite of the different disinhibited behaviors (negative urgency, aggressive behavior, self-harm, and problematic alcohol and drug use) and then the individual behaviors, as moderators in the repeated measures GLMs from Aim 1. Significant 3-way interactions (threat $\mathrm{x}$ congruence $\mathrm{x}$ disinhibition) were followed up by examining zero-order correlations between disinhibited behaviors and our dependent variable of interest within each of the threat and congruence conditions, in order to provide the best representation of our effects.

2.7.3. Supplemental Analyses. In order to account for any potential confounds, several variables were added as covariates across our aims. First, in order to account for any possible order effects, we conducted a 3-way mixed model repeated measures ANCOVA in order to examine interactions between block order (order 1 vs. 2), congruence and threat block (N vs. P vs. $U$ threat block) in the Aim 1 analyses. Next, the level of shock that the participants received during the NPU-ANT task (i.e., the shock the participants rated as "uncomfortable" during the shock sensitivity evaluation) was entered in as a covariate in all Aim 2 analyses. Lastly, because previous research has found that demographic variables such as sex and age are associated with disinhibited behavior and cognitive control (Garcia-Garcia, Domínguez-Borràs, SanMiguel, \& Escera, 2008; Gamboz, Zamarian, \& Cavallero, 2009; Stoet, 2010), and symptoms of anxiety are associated with exaggerated responses to threat cues (Davis et al., 2010), we examined 
associations between our dependent measures and these variables. If significant associations were encountered, we examined these as covariates in analyses of Aim 2.

2.7.4. Power Analysis. A post hoc power analysis was run using G*Power (Faul, Erdfelder, Lang, \& Buchner, 2007). Results determined that given our sample size of 114 participants for the ERP data, an ANCOVA with a within-between interaction and an alpha of .05 would yield .85 power to detect a medium effect size $(f=.25$; Cohen, 1988). Therefore, even the analyses that required the most statistical power (Aim 2 interactions), should have had adequate power to detect a medium size effect but not a small effect. 


\section{CHAPTER THREE:}

\section{RESULTS}

\subsection{Manipulation Check}

On a scale of 0 to 4 , participants rated the shock to be moderately intense $(M=2.10$, $S D=1.0)$, fear/ anxiety provoking $(M=1.56, S D=1.0)$, and mildly painful $(M=1.28, S D=$ 1.33). The average participant reported being quite attentive $(M=3.5, S D=.72)$ and not sleepy throughout the task $(M=1.54, S D=1.26)$. As expected given our design, participants retrospectively reported more fear/ anxiety during conditions of threat (vs. no threat) $F(1,141)=$ $221.46, p<.00, \eta_{\mathrm{p}}^{2}=.18[M(S D):$ threat $=1.87(1.18) ;$ no threat $=.35(.84)]$, and during unpredictable (vs. predictable) threat conditions, $F(1,141)=30.73, p<.00, \eta_{\mathrm{p}}{ }^{2}=.61[M(S D)$ : unpredictable $=2.08(1.22) ;$ predictable $=1.66(1.13)]$

\subsection{Aim 1: Impact of Threat on Cognitive Control}

Grand average flanker-locked ERP waveforms for each condition at frontocentral, central, and parietal sites are illustrated below in Figure 2. Also see Table 2 for a descriptive summary of the physiological and laboratory behavioral data from the NPU-ANT task.

Table 2. Descriptive Summary of ERP and Behavioral Data from NPU-ANT Task.

\begin{tabular}{ccccccc}
\hline & FC N2 & FC P3 & C P3 & P P3 & RT & Accuracy \\
\hline Incongruent & $-0.96(1.57)$ & $2.37(1.95)$ & $3.09(2.19)$ & $4.15(2.45)$ & $545.18(118.91)$ & $96.22(6.37)$ \\
Congruent & $-0.55(1.50)$ & $1.83(1.66)$ & $2.82(1.86)$ & $3.99(2.41)$ & $482.96(113.47)$ & $97.70(4.14)$ \\
Predictable & $-0.87(1.58)$ & $1.90(1.71)$ & $2.73(1.93)$ & $3.87(2.41)$ & $515.65(119.81)$ & $97.88(4.75)$ \\
Unpredictable & $-0.68(1.60)$ & $2.08(1.89)$ & $2.93(2.01)$ & $4.03(2.23)$ & $513.09(116.90)$ & $97.28(5.07)$ \\
No Threat & $-0.68(1.53)$ & $2.13(1.86)$ & $3.02(2.11)$ & $4.28(2.55)$ & $513.55(113.87)$ & $97.05(5.18)$ \\
Threat (P/U) & $-0.78(1.59)$ & $1.99(1.80)$ & $2.83(1.97)$ & $3.95(2.32)$ & $514.37(118.36)$ & $97.58(4.74)$ \\
\hline
\end{tabular}

Note. $F C=$ frontocentral, $C=$ central, $P=$ parietal. Threat $=$ average of predictable and unpredictable threat conditions. ERP data sample: $n=114$, behavioral data sample: $n=134$. 


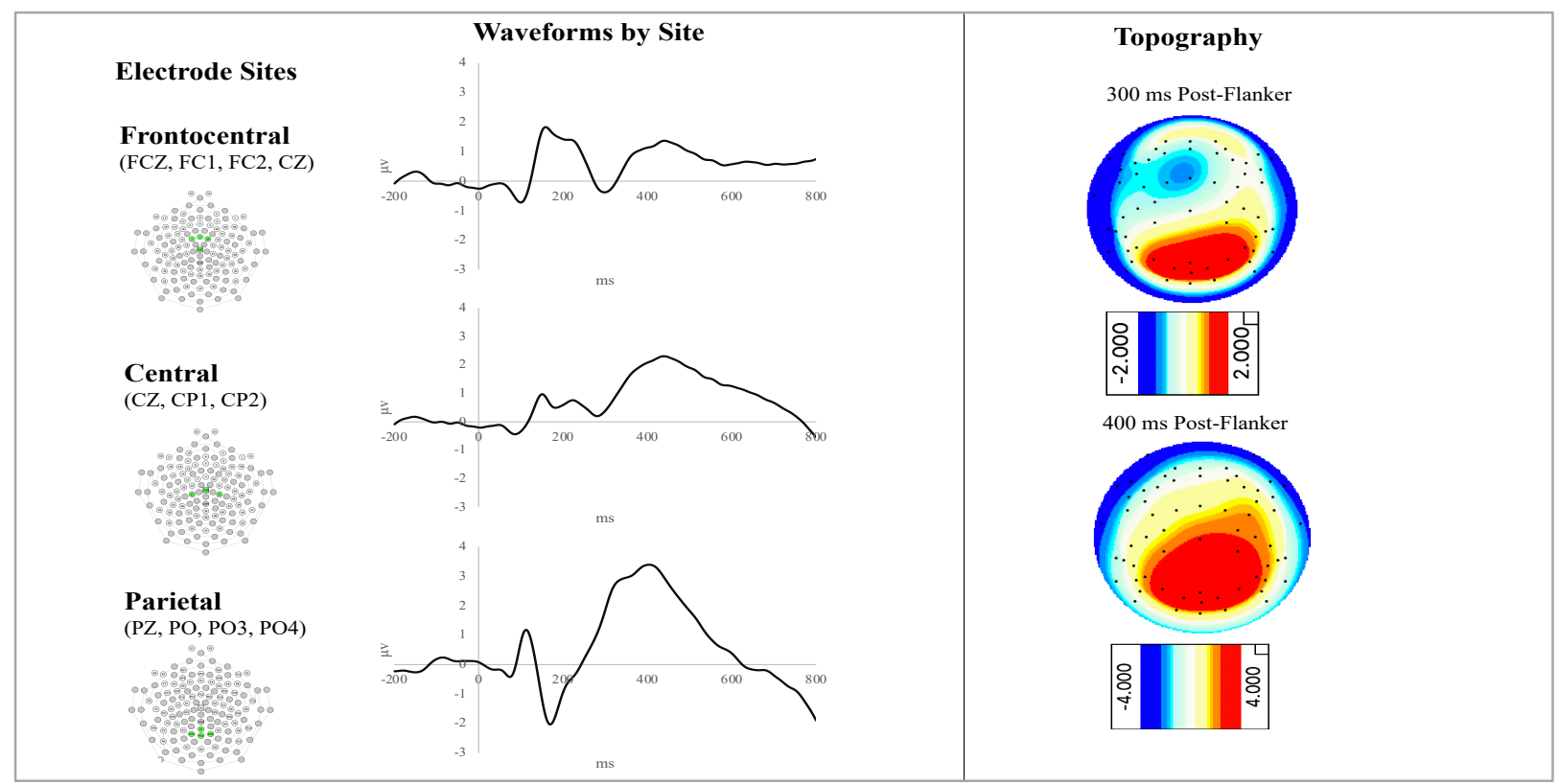

Figure 2. Flanker-Locked ERP Waveforms by Site and Topography.

3.2.1. Analysis of Electrode Site. For the $\underline{N 2}$, visual inspection of the waveforms and scalp topography indicated a clear N2 frontocentrally (see Figure 2). There was a small N2 centrally, but it was not evident in the scalp topography. Given that the N2 effect was clearly maximal and most apparent at the frontocentral site (in both the waveform and the scalp topography) and because we expected the N2 frontocentrally based on previous literature (Brydges et al., 2012), we therefore chose to conduct analyses involving the N2 only frontocentrally. As noted, the frontocentral N2 is said to assess top-down allocation of attention (Heil et al., 2000; Kopp et al., 1996).

For $\underline{P 3}$, visual inspection of the scalp topography and grand average waveform indicated a P3 during our time-window of interest at frontocentral, central, and parietal regions (see Figure 2). Thus, we conducted a series of three-way repeated measures GLMs in order to examine electrode site (ordered in site analysis as frontocentral, parietal, central sites) and its interactions with flanker congruence (congruent, incongruent) and threat condition (ordered as $\mathrm{P}, \mathrm{N}, \mathrm{U})$. A priori polynomial contrasts were used to examine effects of site, with the quadratic contrast 
indicating that P3 amplitude increased or decreased from back to front of head (parietal versus frontocentral/central sites), and the linear contrast representing a difference in $\mathrm{P} 3$ amplitude for the frontocentral versus central region. As noted, threat effects were also examined as a priori contrasts, with an overall threat comparison indicating that $\mathrm{P} 3$ amplitude differed between no threat and threat conditions (i.e., $\mathrm{P} / \mathrm{U}$ vs. $\mathrm{N}$ ) and a predictability contrast indicating that $\mathrm{P} 3$ amplitude differed for unpredictable versus predictable threat conditions (i.e., P vs. U). Results of site analyses are presented in Table 3 below.

Table 3. GLM Effects by P3 Site.

\begin{tabular}{lllll}
\hline & $\boldsymbol{F}$ & $\mathbf{d f}$ & $\boldsymbol{p}$ & $\boldsymbol{\eta}_{\mathrm{p}}{ }^{2}$ \\
\hline Site (Linear) & 60.48 & $(1,113)$ & $.000^{* * *}$ & .34 \\
Site (Quadratic) & 70.22 & $(1,113)$ & $.000^{* * *}$ & .38 \\
Threat Predictability & 7.67 & $(1,113)$ & $.007^{* *}$ & .06 \\
Overall Threat & 12.15 & $(1,113)$ & $.001^{* *}$ & .10 \\
Congruence & 7.46 & $(1,113)$ & $.007^{* *}$ & .06 \\
Linear Site x Threat Predictability & 1.13 & $(1,113)$ & .29 & .01 \\
Linear Site x Overall Threat & 0.34 & $(1,113)$ & .56 & .00 \\
Quadratic Site x Threat Predictability & 0.00 & $(1,113)$ & .96 & .00 \\
Quadratic Site x Overall Threat & 1.69 & $(1,113)$ & .20 & .02 \\
Linear Site x Congruence & 14.38 & $(1,113)$ & $.000^{* * *}$ & .12 \\
Quadratic Site x Congruence & 23.26 & $(1,113)$ & $.000^{* * *}$ & .17 \\
Linear Site x Threat Predictability x Congruence & 0.91 & $(1,113)$ & .34 & .01 \\
Linear Site x Overall Threat x Congruence & 0.10 & $(1,113)$ & .32 & .01 \\
Quadratic Site x Threat Predictability x Congruence & 0.00 & $(1,113)$ & .97 & .00 \\
Quadratic Site x Overall Threat x Congruence & 0.07 & $(1,113)$ & .80 & .00 \\
\hline
\end{tabular}

Note. ${ }^{*} p<.05,{ }^{* *} p<.01,{ }^{* * *} p<.001$.

Our results indicated that there were significant linear (frontocentral vs. central) and quadratic (parietal vs. frontocentral/ central) effects of site, such that P3 amplitude increased moving from the front to the back of head and was largest at parietal sites. There were no Threat $\mathrm{x}$ Site interactions, suggesting that the effect of threat condition on P3 amplitude did not significantly vary by site. However, we did find that conflict processing varied slightly by site. Specifically, results indicated both a significant linear site (frontocentral vs. central) $\mathrm{x}$ congruence as well as quadratic site (parietal vs. frontocentral/ central) x congruence 
interactions, such that the flanker congruence differentiation was maximal frontocentrally (compared to centrally and parietally; see Figure 3-with congruence effect at frontocentral site highlighted).

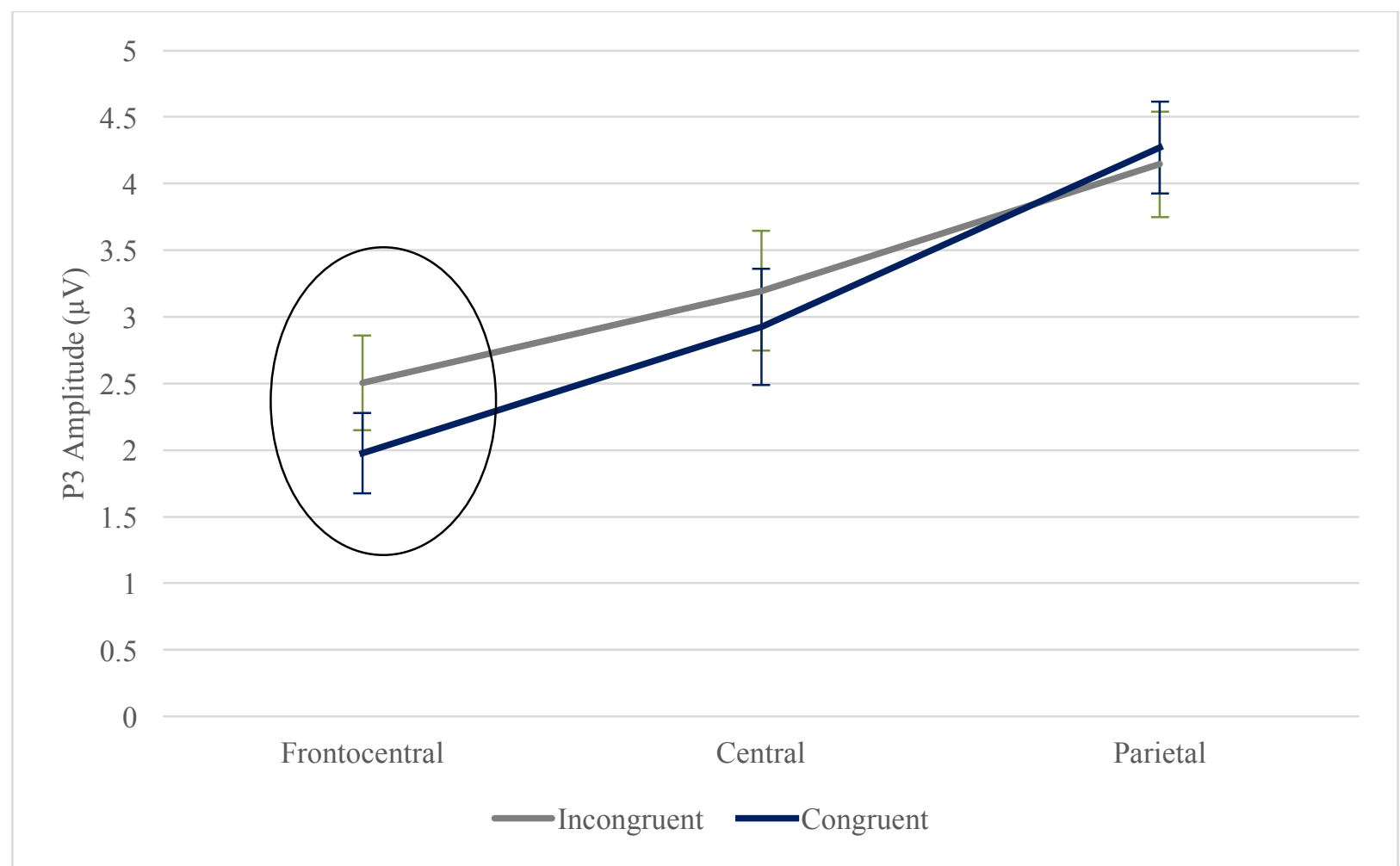

Figure 3. Amplitude of the P3 Responses to Flanker Congruence by Site.

Together these findings are in line with previous P3 research, specifically that the P3 was maximal parietally but the conflict processing effect was maximal frontocentrally (Rietdijk, Franken, \& Thurik, 2014; Xie, Ren, Cao, \& Li, 2017). This is consistent with prior distinction between the frontal P3a (i.e., attention-switching) and the parietal P3b (i.e., inhibition; Polich, 2007). Given our focus on inhibition and recent research which questions whether the conflict N2 and the conflict P3 reflect distinct components (Kamala, Szewczyk, Senderecka, \& Wodniecka, 2017), we decided to focus on parietal P3, our a-priori component of interest, in the main text. However, for completeness we have also included analyses involving the frontocentral and central P3 in the supplemental materials. 
3.2.2. Threat and Cognitive Control: ERP Results. For Aim 1, we conducted a series of mixed-model repeated measures Threat x Congruence GLMs on frontocentral N2 and parietal P3. Again, we focused on a priori polynomial contrasts of threat, specifically threat (un)predictability ( $P$ vs. $U$ ) and overall effect of threat (vs. no threat; $P / U$ vs. $N$ ). See Table 4 for results of these GLMs and refer to Table 2 for condition means. See Figure 4 for Grand average waveforms and scalp topographies for each condition at frontocentral and parietal sites.

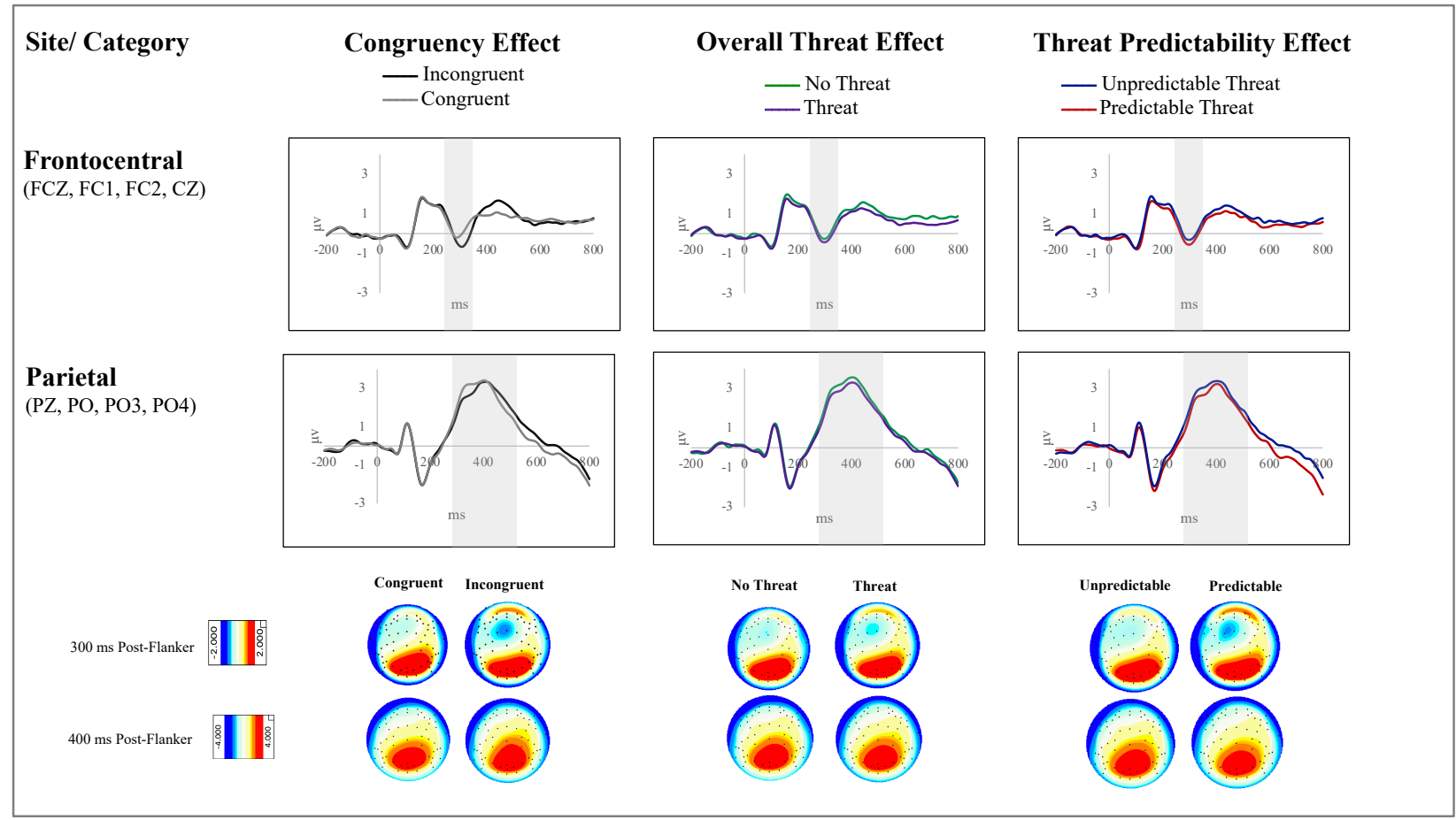

Figure 4. ERP Waveforms and Topographies by Condition and Site.

Frontocentral N2 results yielded the expected, large effect of flanker congruence, $F(1$, $113)=17.62, p<.001, \eta^{2}=0.14$, such that the $\mathrm{N} 2$ was larger (more negative) for incongruent vs. congruent flanker stimuli. There was also an effect of threat predictability (P vs. U) on frontocentral $\mathrm{N} 2$ amplitude, $F(1,137)=3.87, p=.05, \eta 2=0.03$, such that the $\mathrm{N} 2$ was larger (more negative) to the flanker during predictable threat contexts, relative to unpredictable threat contexts (and neutral; see Table 2), suggesting enhanced early attentional when exposed to relatively predictable vs. unpredictable threat. Effects of the overall threat contrast and Threat $\mathrm{x}$ 
Congruence interactions were not significant, indicating that, contrary to our hypotheses, the congruence effect did not differ as a function of threat condition for N2.

$\underline{\text { Parietal } P 3}$ analyses revealed a significant overall effect of threat (P/U vs. N), $F(1$, $113)=7.48, p<.001, \eta 2=0.06$, such that the $\mathrm{P} 3$ was smaller (less positive) during threat versus no threat contexts. These results are in line with our expectations that P3 amplitude would be blunted under conditions of threat. In addition to the effect of overall threat, effects of threat (un)predictability (P vs. $\mathrm{U}$ ) were also present, $F(1,113)=10.23, p=.04, \eta 2=0.08$ ), such that the $\mathrm{P} 3$ amplitude was especially reduced less positive) during predictable (vs. unpredictable) threat contexts - opposite of the flanker N2 effects above ${ }^{4}$. Finally, our findings did not reveal any effect of flanker congruence at the parietal P3 site, $F(1,113)=7.90, p=.23, \eta 2=0.07$, although the congruence effect was fairly robust at frontocentral and central P3 sites (see supplemental material for more information).

Table 4. ERP GLM Effects.

\begin{tabular}{lllll}
\hline & $\boldsymbol{F}$ & $\mathbf{d f}$ & $\boldsymbol{p}$ & $\boldsymbol{\eta}_{\mathbf{p}}{ }^{2}$ \\
\hline Frontocentral N2 & & & & \\
Threat Predictability & 3.87 & $(1,113)$ & $.05^{\wedge}$ & .03 \\
Overall Threat & 1.47 & $(1,113)$ & .23 & .01 \\
Congruence & 17.62 & $(1,113)$ & $.00^{* * *}$ & .14 \\
Threat Predictability x Congruence & 0.94 & $(1,113)$ & .37 & .01 \\
Overall Threat x Congruence & 1.88 & $(1,113)$ & .17 & .02 \\
\hline Parietal P3 & & & & \\
Threat Predictability & 10.23 & $(1,113)$ & $.04^{*}$ & .08 \\
Overall Threat & 7.48 & $(1,113)$ & $.00^{* * *}$ & .06 \\
Congruence & 7.90 & $(1,113)$ & .23 & .07 \\
Threat Predictability x Congruence & .00 & $(1,113)$ & .81 & .00 \\
Overall Threat x Congruence & 3.01 & $(1,113)$ & .23 & .03 \\
\hline
\end{tabular}

Note. ${ }^{*} p<.05,{ }^{* *} p<.01,{ }^{* * *} p<.001, \wedge$ marginal effect $(p>.05)$.

In sum, ERP analyses revealed the expected effect of flanker congruence (greater incongruent vs. congruent processing) at frontocentral N2, indicative of conflict processing. We

\footnotetext{
${ }^{4}$ Threat effects were consistent across all P3 sites. See Appendix O (supplemental material) for more information.
} 
also found that threat condition altered the degree of processing of flanker stimuli (regardless of congruence trial type). Specifically, we observed a pattern of enhanced (less negative) N2 during predictable (vs. unpredictable) threat, followed by a relatively blunted (less positive) P3 during overall threat (vs. no threat), although more so for predictable versus unpredictable threat. Finally, both frontocentral N2 and parietal P3 results suggested that conflict processing (i.e. differential processing of flanker congruence trial types) did not markedly vary as a function of threat condition.

\subsubsection{Threat and Cognitive Control: Behavioral Results. Behavioral measures of} performance on the NPU-ANT task including accuracy (i.e., total percentage of correct trials) and RT were also analyzed as a function of threat and congruence. See Table 5 for results of these GLMs and refer to Table 2 for condition means.

For $\underline{R T}$, a large main effect of flanker congruence was observed, $F(1,137)=565.52, p$ $<.001, \eta 2=0.81$, such that, as expected, participants were slower to respond to incongruent versus congruent flanker stimuli. Analyses did not reveal any significant impact of threat contrasts on reaction time.

For Accuracy, results indicated that the task was fairly easy, given high rates of accuracy across participants $(M \%(S D)=97.40(4.74))$. As expected, participants were less accurate during incongruent (vs. congruent) flanker trials, $F(1,137)=37.53, p<.001, \eta 2=0.22$. Analyses also revealed both an overall effect of threat $(\mathrm{P} / \mathrm{U}$ vs. $\mathrm{N} ; F(1,137)=6.05, p=.02, \eta 2=0.04)$, as well as an effect of threat predictability (P vs. $\mathrm{U} ; F(1,137)=7.75, p=.01, \eta 2=0.05)$. Although participants showed greater accuracy during conditions of threat, this was due primarily to predictable (vs. unpredictable) threat, whereas accuracy was similarly poor during unpredictable threat and neutral conditions. However, the latter main effect of predictability was dependent on 
a significant Threat Predictability x Congruence interaction (P vs. U; $F(1,137)=1.27, p=.02, \eta 2$ $=0.04)$. Specifically, the poorer accuracy for incongruent vs. congruent trials was greater under unpredictable vs. predictable threat blocks (see Figure 5). This result is in line with predictions that unpredictable threat would impact cognitive control processes, including increasing interference by flanker congruence.

Table 5. Behavioral GLM Effects.

\begin{tabular}{lllll}
\hline & $\boldsymbol{F}$ & $\mathbf{d f}$ & $\boldsymbol{p}$ & $\boldsymbol{\eta}_{\mathbf{p}}{ }^{2}$ \\
\hline Reaction Time & & & & \\
Threat Predictability & .79 & $(1,137)$ & .38 & .01 \\
Overall Threat & .13 & $(1,137)$ & .72 & .00 \\
Congruence & 565.52 & $(1,137)$ & $.00^{* * *}$ & .81 \\
Threat Predictability x Congruence & .44 & $(1,137)$ & .51 & .00 \\
Overall Threat x Congruence & .94 & $(1,137)$ & .34 & .01 \\
\hline Accuracy & & & & \\
Threat Predictability & 7.75 & $(1,137)$ & $.01^{* *}$ & .05 \\
Overall Threat & 6.05 & $(1,137)$ & $.02^{*}$ & .04 \\
Congruence & 37.53 & $(1,137)$ & $.00^{* * *}$ & .22 \\
Threat Predictability x Congruence & 5.48 & $(1,137)$ & $.02^{*}$ & .04 \\
Overall Threat x Congruence & 1.27 & $(1,137)$ & .26 & .01 \\
\hline
\end{tabular}

Note. ${ }^{*} p<.05,{ }^{* *} p<.01, * * * p<.001$.

Together, these behavioral analyses revealed the expected flanker task effects of slower RT and poorer accuracy to incongruent versus congruent trials. Although we did not expect participants to demonstrate improved accuracy, including during incongruent trials, in the threat conditions, this was present mainly during predictable threat. The unpredictable threat condition was marked by poorer accuracy than the predictable condition, and similar to the neutral blocks.

In sum, results across ERP and behavioral measures indicated that compared to predictable threat contexts, relatively unpredictable threat contexts were associated with decreased early processing (blunted N2) and decreased accuracy during incongruent trials. Interestingly, in some cases, predictable threat was associated with heightened processing and improved accuracy, even compared to neutral blocks (see Figure 5). 


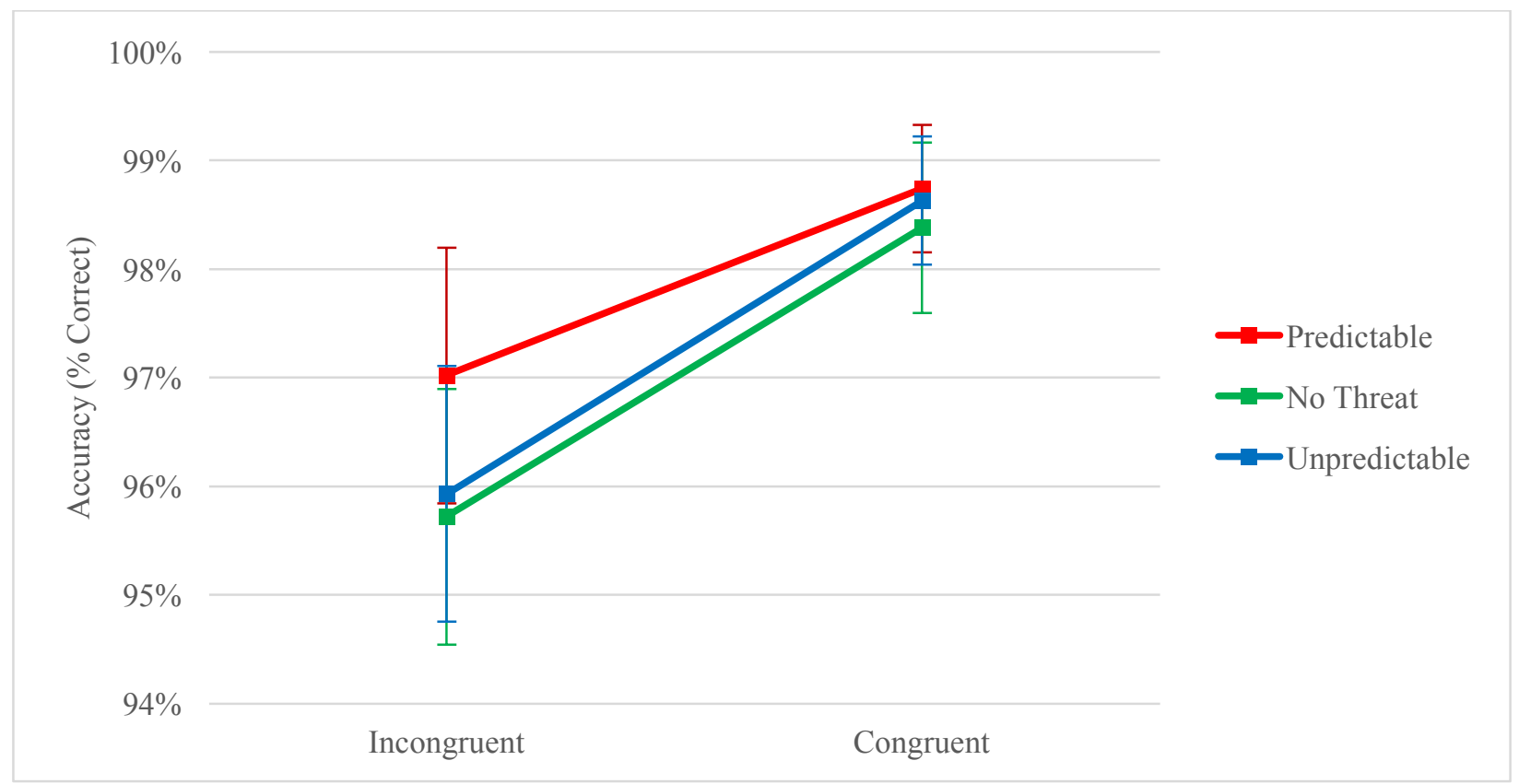

Figure 5. Accuracy as a Function of Flanker Congruence and Threat Condition.

\subsection{Aim 2: Associations with Disinhibited Behavior}

For Aim 2, we added disinhibited behavior as a continuous between-subjects factor in the repeated measures GLMs conducted in Aim 1 above. First, a disinhibition factor representing a shared liability across the variables (including negative urgency, alcohol and substance use disorder symptoms, self-harm, and aggressive behavior) was extracted through factor analysis and included in subsequent analyses. Second, separate analyses were conducted for each disinhibited behavior variable. Interactions involving disinhibition were clarified by examining the magnitude and direction of associations between neurocognitive and task behavioral measures and disinhibited behaviors. Descriptive information and bivariate correlations for measures of disinhibited traits and behaviors are displayed in Table 6 .

Table 6. Descriptive Statistics and Associations Among Disinhibited Behaviors.

\begin{tabular}{|c|c|c|c|c|c|c|}
\hline & 1. & 2. & 3. & 4. & 5. & 6. \\
\hline 1. Disinhibited Behavior Factor & -- & -- & -- & -- & -- & -- \\
\hline 2. Negative Urgency & $.63 * * *$ & -- & -- & -- & -- & -- \\
\hline 3. Aggressive Behavior & $.78 * * *$ & $.36^{* * *}$ & -- & -- & -- & -- \\
\hline 4. Self-harm & $.58 * * *$ & $.26 * * *$ & $.33 * * *$ & -- & -- & -- \\
\hline
\end{tabular}


Table 6. (Continued)

\begin{tabular}{lllllll}
\hline 5. Alcohol Use Symptoms & $.58^{* * *}$ & $.23^{* *}$ & $.28^{* *}$ & $.22^{*}$ & -- & -- \\
6. Substance Use Symptoms & $.67^{* * *}$ & $.27^{* *}$ & $.35^{* * *}$ & $.28^{* *}$ & $.32^{* * *}$ & -- \\
\hline$M$ & -.01 & 25.29 & 9.27 & .16 & 3.39 & 3.31 \\
\hline$S D$ & .82 & 9.02 & 4.95 & .27 & 3.31 & 3.72 \\
\hline Range & $-1.51-1.94$ & $12-48$ & $0-23$ & $0-.95$ & $0-11$ & $0-11$ \\
\hline Skewness & .41 & .65 & .27 & 1.41 & .73 & .76 \\
\hline Kurtosis & .21 & -.19 & -.28 & .45 & -.68 & -.81 \\
\hline$N$ & 138 & 138 & 143 & 143 & 143 & 143 \\
\hline
\end{tabular}

Note $-{ }^{*} p<.05,{ }^{* *} p<.01,{ }^{* * *} p<.001$. Note - self-harm was log transformed.

\subsubsection{Disinhibited Behavior Factor Results. In order to examine whether associations}

involving disinhibited behaviors are characteristic of specific manifestations, or disinhibited proneness more generally, we first used an Exploratory Factor Analysis (EFA) to estimate a general disinhibition factor, representing shared variance across disinhibited behaviors. Results of the factor analysis indicated that all items loaded onto a single general Disinhibition factor (43.06\% total variance explained), as indicated by both scree plot and eigen values, with standardized loadings ranging from .47 to .65 (see Table 7). The latent Disinhibition factor scores were extracted for use in subsequent analyses.

Table 7. Standardized factor loadings of the Disinhibited Behaviors EFA.

\begin{tabular}{lc}
\hline Item & Disinhibition Factor Loadings \\
\hline UPPSP - Negative Urgency & .52 \\
LHA - Aggression & .65 \\
LHA - Self-Harm & .48 \\
MINI - Alcohol Use Disorder symptoms & .47 \\
MINI - Substance Use Disorder symptoms & .56 \\
\hline
\end{tabular}

Analyses of the frontocentral N2 revealed no effects involving the Disinhibition factor (see Table 8). However, at the parietal P3 site $^{5}$, there was both a two-way Disinhibition $\mathrm{x}$ Overall Threat $(\mathrm{P} / \mathrm{U}$ vs. $\mathrm{N} ; F(1,109)=4.32, p=.04, \eta 2=0.04)$ interaction, which was superseded by a three-way Disinhibition x Overall Threat (P/U vs. N) x Congruence interaction,

\footnotetext{
${ }^{5}$ Disinhibition was also unrelated to P3 amplitude at frontocentral and central sites.
} 
$F(1,109)=5.20, p=.03, \eta 2=0.05$. Follow up analyses showed that the Disinhibition $\mathrm{x}$

Congruence interaction was not significant within each threat condition (threat: $F(1,109)=1.57$, $p=.21, \eta_{\mathrm{p}}{ }^{2}=.01$; no threat: $\left.F(1,109)=2.29 p=.13, \eta_{\mathrm{p}}{ }^{2}=.02\right)$. Instead, the pattern of correlations was consistent with a cross-over interaction, such that Disinhibition scores were related to decreased parietal P3 incongruent - congruent difference score during no threat $(r=-.14, p=.13)$ and greater congruence differentiation during overall threat $(r=.12, p=.21)$. See Figure 6 for an illustration. The latter result suggests that the expected disinhibition-related reductions in congruence $\mathrm{P} 3$ seem to be corrected during conditions of threat.

Table 8. GLM Effects of ERPs as a function of Disinhibition Composite.

\begin{tabular}{lllll}
\hline & $\boldsymbol{F}$ & $\mathbf{d f}$ & $\boldsymbol{p}$ & $\boldsymbol{\eta}_{\mathbf{p}}{ }^{2}$ \\
\hline Frontocentral Site - N2 & & & & \\
\hline Threat Predictability & 3.91 & $(1,109)$ & $.05^{\wedge}$ & .04 \\
Overall Threat & 1.50 & $(1,109)$ & .24 & .01 \\
Congruence & 20.47 & $(1,109)$ & $.00^{* * *}$ & .16 \\
Disinhibition (Between Subjects) & .65 & $(1,109)$ & .42 & .01 \\
Threat Predictability x Disinhibition & 1.36 & $(1,109)$ & .25 & .01 \\
Overall Threat x Disinhibition & 2.68 & $(1,109)$ & .10 & .02 \\
Congruence x Disinhibition & .07 & $(1,109)$ & .79 & .00 \\
Threat Predictability x Congruence & .74 & $(1,109)$ & .39 & .01 \\
Overall Threat x Congruence & 2.44 & $(1,109)$ & .12 & .02 \\
Threat Predictability x Congruence x Disinhibition & .04 & $(1,109)$ & .84 & .00 \\
Overall Threat x Congruence x Disinhibition & .42 & $(1,109)$ & .52 & .00 \\
\hline Parietal Site - P3 & & & & \\
\hline Threat Predictability & 3.88 & $(1,109)$ & $.05^{\wedge}$ & .03 \\
Overall Threat & 13.44 & $(1,109)$ & $.00^{* * *}$ & .11 \\
Congruence & 2.13 & $(1,109)$ & .15 & .02 \\
Disinhibition (Between Subjects) & .18 & $(1,109)$ & .68 & .00 \\
Threat Predictability x Disinhibition & .42 & $(1,109)$ & .52 & .00 \\
Overall Threat x Disinhibition & 4.32 & $(1,109)$ & $.04^{*}$ & .04 \\
Congruence x Disinhibition & .05 & $(1,109)$ & .83 & .00 \\
Threat Predictability x Congruence & .17 & $(1,109)$ & .68 & .00 \\
Overall Threat x Congruence & .36 & $(1,109)$ & .55 & .00 \\
Threat Predictability x Congruence x Disinhibition & .01 & $(1,109)$ & .93 & .00 \\
Overall Threat x Congruence x Disinhibition & 5.20 & $(1,109)$ & $.03 *$ & .05 \\
\hline
\end{tabular}

Note. ${ }^{*} p<.05,{ }^{* *} p<.01,{ }^{* * *} p<.001,{ }^{\wedge}$ marginal effect $(p>.05)$. 


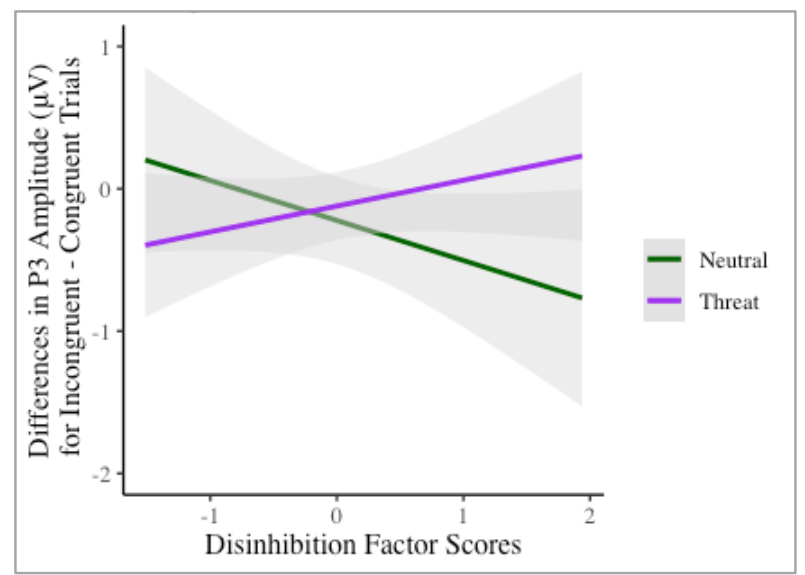

Figure 6. Disinhibition x Overall Threat x Congruence Interaction at Parietal P3.

For the behavioral measures, analyses of $\underline{R T}$ revealed no effects involving Disinhibition, whereas analyses of accuracy revealed a three-way Disinhibition x Overall Threat x Congruence interaction, $F(1,131)=4.04, p<.05, \eta 2=0.03$ (see Table 9). Follow up analyses revealed that the Disinhibition x Congruence interaction was not significant within each threat condition (Threat: $F(1,131)=1.24, p=.27, \eta_{\mathrm{p}}{ }^{2}=.01$; No Threat: $\left.F(1,131)=0.58 p=.45, \eta_{\mathrm{p}}{ }^{2}=.00\right)$. Instead, as seen in with the P3, the nature of this interaction was a cross-over interaction, showing increases in accuracy for incongruent trials with higher scores on Disinhibition during overall threat $(r=$ $.10, p=.27)$ and small negative association during no threat $(r=-.07, p=.45)$. See Figure 7 .

Table 9. GLM Effects of Behavior as a function of Disinhibition Composite.

\begin{tabular}{lllll}
\hline Reaction Time & $\boldsymbol{F}$ & $\mathbf{d f}$ & $\boldsymbol{p}$ & $\mathbf{\eta}_{\mathbf{p}}{ }^{\mathbf{2}}$ \\
\hline Threat Predictability & & & & \\
Overall Threat & .54 & $(1,131)$ & .46 & .00 \\
Congruence & .26 & $(1,131)$ & .61 & .00 \\
Disinhibition (Between Subjects) & 558.82 & $(1,131)$ & $.00 * * *$ & .81 \\
Threat Predictability x Disinhibition & .29 & $(1,131)$ & .59 & .00 \\
Overall Threat x Disinhibition & .01 & $(1,131)$ & .94 & .00 \\
Congruence x Disinhibition & .13 & $(1,131)$ & .72 & .00 \\
Threat Predictability x Congruence & 1.58 & $(1,131)$ & .21 & .01 \\
Overall Threat x Congruence & .56 & $(1,131)$ & .46 & .00 \\
Threat Predictability x Congruence x Disinhibition & 1.46 & $(1,131)$ & .23 & .01 \\
Overall Threat x Congruence x Disinhibition & 1.49 & $(1,131)$ & .23 & .01 \\
\hline Accuracy & .00 & $(1,131)$ & .98 & .00 \\
\hline Threat Predictability & & & & \\
\hline
\end{tabular}


Table 9. (Continued)

\begin{tabular}{lllll} 
Overall Threat & 5.36 & $(1,131)$ & $.02^{*}$ & .04 \\
Congruence & 35.52 & $(1,131)$ & $.00^{* * *}$ & .21 \\
Disinhibition (Between Subjects) & .58 & $(1,131)$ & .45 & .00 \\
Threat Predictability x Disinhibition & .29 & $(1,131)$ & .59 & .00 \\
Overall Threat x Disinhibition & 1.54 & $(1,131)$ & .22 & .01 \\
Congruence x Disinhibition & .36 & $(1,131)$ & .55 & .00 \\
Threat Predictability x Congruence & 5.23 & $(1,131)$ & $.02^{*}$ & .04 \\
Overall Threat x Congruence & 1.24 & $(1,131)$ & .27 & .01 \\
Threat Predictability x Congruence x Disinhibition & 1.73 & $(1,131)$ & .19 & .01 \\
Overall Threat x Congruence x Disinhibition & 4.04 & $(1,131)$ & $.05^{*}$ & .03 \\
\hline
\end{tabular}

Note. ${ }^{*} p<.05,{ }^{* *} p<.01,{ }^{* * *} p<.001$.

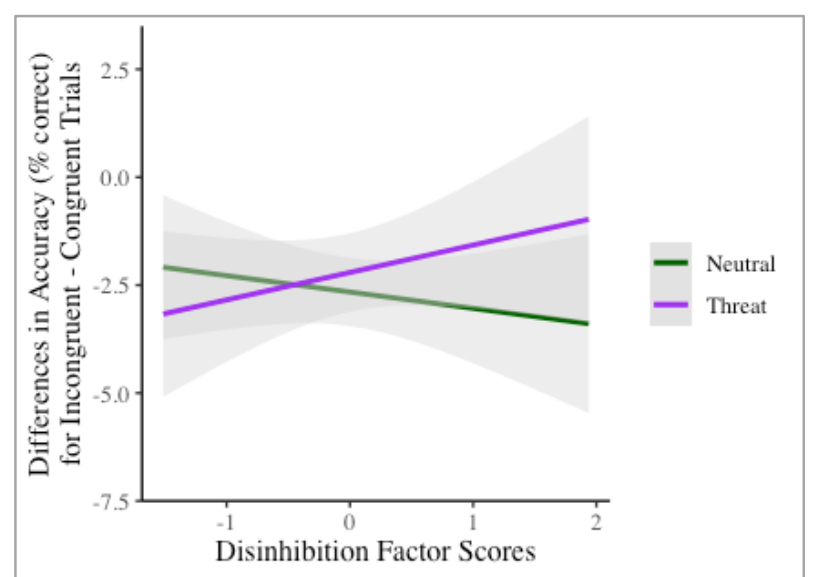

Figure 7. Disinhibition x Overall Threat x Congruence Interaction of Accuracy.

In sum, the Disinhibition factor was associated with increases in the P3 and improved accuracy to incongruent vs. congruent trials during overall threat vs. no threat conditions. However, these effects were relatively small in size and their meaningfulness is unclear.

\subsubsection{Negative Urgency Results. Analyses of the $\underline{P 3}$ revealed no effects involving} negative urgency ${ }^{6}$, whereas analyses of the $\underline{N 2}$ revealed both a significant two-way Negative Urgency x Congruence interaction, $F(1,109)=5.57, p=.02, \eta_{\mathrm{p}}{ }^{2}=.05$ and a small, marginally significant two-way Negative Urgency x Overall Threat (P/U vs. N) interaction, $F(1,109)=2.90$, $p=.09, \eta_{\mathrm{p}}^{2}=.03$. See Table 10. First, we decomposed the two-way Negative Urgency $\mathrm{x}$

\footnotetext{
${ }^{6}$ However, we did observe an Overall Threat $\mathrm{x}$ Negative Urgency effect at both frontocentral and central P3 sites, such that higher scores of Negative Urgency were associated with reduced P3 processing during threat and enhanced $\mathrm{P} 3$ processing during no threat. Refer to Appendix O (supplemental materials) for more information.
} 
Congruence interaction, which showed that increases in negative urgency were associated with decreases in N2 congruence differentiation (see Figure 8). Next, decomposition of the marginally significant two-way Negative Urgency x Overall Threat (P/U vs. N) interaction indicated that negative urgency was associated with increasing (more negative) N2 during situations of threat $(r=-.05, p=.64)$ and decreasing (less negative) $\mathrm{N} 2$ during no threat $(r=.03, p=.73)$, resulting in a greater N2 threat effect with higher scores on negative urgency. See Figure 9.

Table 10. GLM Effects of ERPs as a function of NU.

\begin{tabular}{lllll}
\hline & $\boldsymbol{F}$ & $\mathbf{d f}$ & $\boldsymbol{p}$ & $\boldsymbol{\eta}_{\mathbf{p}}{ }^{\mathbf{2}}$ \\
\hline Frontocentral Site - N2 & & & & \\
\hline Threat Predictability & .249 & $(1,109)$ & .62 & .00 \\
Overall Threat & 1.38 & $(1,109)$ & .24 & .01 \\
Congruence & 14.12 & $(1,109)$ & $.00^{* * *}$ & .12 \\
NU (Between Subjects) & .06 & $(1,109)$ & .81 & .00 \\
Threat Predictability x NU & .03 & $(1,109)$ & .86 & .00 \\
Overall Threat x NU & 2.9 & $(1,109)$ & $.09^{\wedge}$ & .03 \\
Congruence x NU & 5.57 & $(1,109)$ & $.02^{*}$ & .05 \\
Threat Predictability x Congruence & .50 & $(1,109)$ & .48 & .01 \\
Overall Threat x Congruence & .27 & $(1,109)$ & .60 & .00 \\
Threat Predictability x Congruence x NU & .19 & $(1,109)$ & .66 & .00 \\
Overall Threat x Congruence x NU & .00 & $(1,109)$ & .97 & .00 \\
\hline Parietal Site - P3 & & & & \\
\hline Threat Predictability & .03 & $(1,109)$ & .87 & .00 \\
Overall Threat & 1.22 & $(1,109)$ & .27 & .01 \\
Congruence & 1.57 & $(1,109)$ & .21 & .01 \\
NU (Between Subjects) & 1.12 & $(1,109)$ & .30 & .01 \\
Threat Predictability x NU & .27 & $(1,109)$ & .60 & .00 \\
Overall Threat x NU & .00 & $(1,109)$ & .96 & .00 \\
Congruence x NU & .67 & $(1,109)$ & .42 & .01 \\
Threat Predictability x Congruence & .00 & $(1,109)$ & .96 & .00 \\
Overall Threat x Congruence & 1.60 & $(1,109)$ & .21 & .02 \\
Threat Predictability x Congruence x NU & .05 & $(1,109)$ & .84 & .00 \\
Overall Threat x Congruence x NU & 2.52 & $(1,109)$ & .12 & .02 \\
\hline Note & & & &
\end{tabular}

Note. ${ }^{*} p<.05,{ }^{* *} p<.01,{ }^{* * *} p<.001,{ }^{\wedge}$ marginal effect $(p>.05)$. 


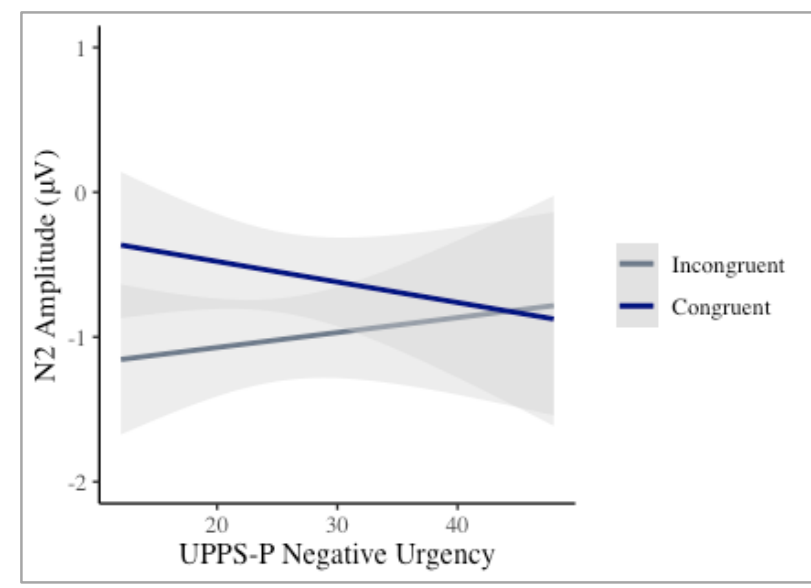

Figure 8. Negative Urgency x Congruence Interaction at Frontocentral N2.

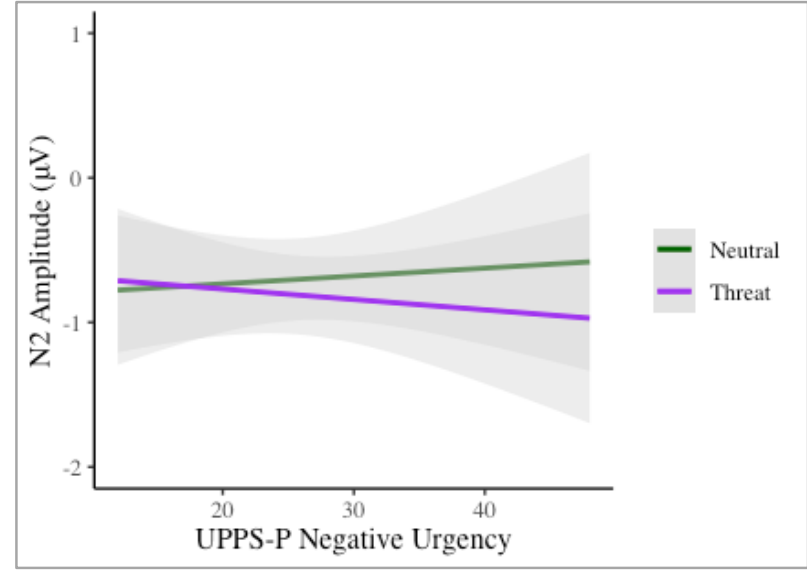

Figure 9. Negative Urgency x Overall Threat Interaction at Frontocentral N2.

For the behavioral measures, analyses of $\underline{R T}$ revealed no effects involving negative urgency, whereas analyses of accuracy revealed a small, marginally significant two-way Negative Urgency x Overall Threat $\left(\mathrm{P} / \mathrm{U}\right.$ vs. N) interaction, $F(1,131)=2.87, p=.09, \eta_{\mathrm{p}}{ }^{2}=.02$ (see Table 11). Follow up analyses revealed that higher levels of negative urgency were associated with slightly decreased accuracy during no threat $(r=-.07, p=.41)$ but not during threat $(r=-.00, p=.99$; see Figure 10$)$.

Table 11. GLM Effects of Behavior as a function of NU.

\begin{tabular}{lllll}
\hline & $\boldsymbol{F}$ & $\mathbf{d f}$ & $\boldsymbol{p}$ & $\boldsymbol{\eta}_{\mathbf{p}}{ }^{2}$ \\
\hline Reaction Time & & & & \\
\hline Threat Predictability & .00 & $(1,131)$ & .97 & .00 \\
Overall Threat & .182 & $(1,131)$ & .67 & .00 \\
Congruence & 79.13 & $(1,131)$ & $.00 * * *$ & .38 \\
NU (Between Subjects) & .01 & $(1,131)$ & .92 & .00
\end{tabular}


Table 11. (Continued)

\begin{tabular}{lllll}
\hline Threat Predictability x NU & .05 & $(1,131)$ & .83 & .00 \\
Overall Threat x NU & .41 & $(1,131)$ & .53 & .00 \\
Congruence x NU & 1.15 & $(1,131)$ & .29 & .01 \\
Threat Predictability x Congruence & 1.88 & $(1,131)$ & .17 & .01 \\
Overall Threat x Congruence & .48 & $(1,131)$ & .49 & .00 \\
Threat Predictability x Congruence x NU & 1.40 & $(1,131)$ & .24 & .01 \\
Overall Threat x Congruence x NU & .09 & $(1,131)$ & .76 & .00 \\
\hline Accuracy & & & & \\
\hline Threat Predictability & 1.21 & $(1,131)$ & .27 & .01 \\
Overall Threat & 0.68 & $(1,131)$ & .41 & .01 \\
Congruence & 8.73 & $(1,131)$ & $.00^{* * *}$ & .06 \\
NU (Between Subjects) & .09 & $(1,131)$ & .77 & .00 \\
Threat Predictability x NU & .08 & $(1,131)$ & .78 & .00 \\
Overall Threat x NU & 2.87 & $(1,131)$ & $.09^{\wedge}$ & .02 \\
Congruence x NU & 1.03 & $(1,131)$ & .31 & .01 \\
Threat Predictability x Congruence & .74 & $(1,131)$ & .39 & .01 \\
Overall Threat x Congruence & 1.12 & $(1,131)$ & .28 & .01 \\
Threat Predictability x Congruence x NU & .01 & $(1,131)$ & .91 & .00 \\
Overall Threat x Congruence x NU & 2.36 & $(1,131)$ & .13 & .02 \\
\hline
\end{tabular}

Note. ${ }^{*} p<.05,{ }^{* *} p<.01,{ }^{* * *} p<.001,{ }^{\wedge}$ marginal effect $(p>.05)$.

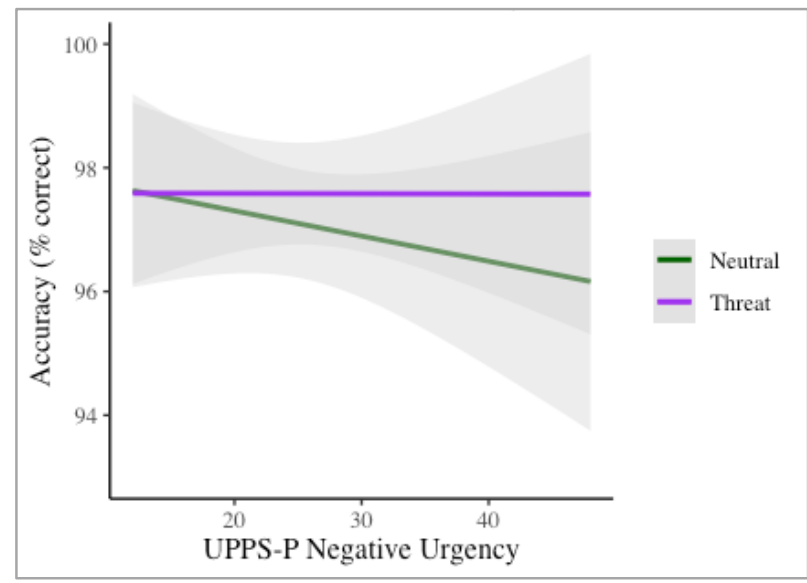

Figure 10. Negative Urgency x Overall Threat Interaction of Accuracy.

These findings are somewhat consistent with our ERP findings that high scorers on negative urgency showed more engagement (more negative N2) with flankers during threat, which resulted in less disruption of accuracy during threat.

3.3.3. Aggression Results. $N 2$ analyses revealed a marginally significant two-way Aggression $\mathrm{x}$ Threat Predictability (P vs. U) interaction, $F(1,112)=4.73, p=.05, \eta_{\mathrm{p}}{ }^{2}=.03$ (see 
Table 12). Follow up correlations indicated that lifetime aggressive behaviors were unrelated to N2 during unpredictable threat, $(r=-.01, p=.91)$, but associated with increasing (more negative) $\mathrm{N} 2$ during conditions of predictable threat $(r=-.14)$, see Figure 11.

Analyses of the $\underline{P 3}$ revealed both a significant two-way Aggression x Overall Threat interaction $(\mathrm{P} / \mathrm{U}$ vs. $\mathrm{N} ; F(1,112)=4.76, p=.03, \eta 2=0.04)$, as well as a marginally significant three-way Aggression $x$ Overall Threat $(\mathrm{P} / \mathrm{U}$ vs. $\mathrm{N}) \mathrm{x}$ Congruence interaction $(F(1,112)=3.23$, $p=.08, \eta 2=0.03)^{7}$. The two-way Aggression x Overall Threat interaction for P3 indicated that aggression was associated with decreased (less positive) P3 amplitude during no threat conditions $(r=-.12, p=.22)$ compared to threat $(\mathrm{r}=-.05)$, resulting in the $\mathrm{P} 3$ threat effect decreasing at higher levels of aggression (see Figure 12). Next, the marginally significant threeway Aggression x Overall Threat x Congruence interaction revealed that higher levels of aggressive behaviors were associated with less parietal P3 differentiation of incongruent and congruent flankers during no threat $(r=-.20, p=.03), F(1,112)=4.84, p=.03, \eta_{\mathrm{p}}{ }^{2}=.04$, but not during threat $(r=-.01, p=.90)$. See Figure 12 .

Table 12. GLM Effects of ERPs as a function of Aggressive Behavior.

\begin{tabular}{lllll}
\hline & $\boldsymbol{F}$ & $\mathbf{d f}$ & $\boldsymbol{p}$ & $\mathbf{\eta}_{\mathbf{p}}{ }^{2}$ \\
\hline Frontocentral Site - N2 & & & & \\
\hline Threat Predictability & .66 & $(1,112)$ & .42 & .01 \\
Overall Threat & .69 & $(1,112)$ & .41 & .01 \\
Congruence & 3.8 & $(1,112)$ & $.05^{\wedge}$ & .03 \\
Aggression (Between Subjects) & .26 & $(1,112)$ & .61 & .00 \\
Threat Predictability x Aggression & 4.73 & $(1,112)$ & $.05^{\wedge}$ & .03 \\
Overall Threat x Aggression & 2.06 & $(1,112)$ & .12 & .02 \\
Congruence x Aggression & .00 & $(1,112)$ & .98 & .00 \\
Threat Predictability x Congruence & .14 & $(1,112)$ & .71 & .00 \\
Overall Threat x Congruence & .31 & $(1,112)$ & .58 & .00 \\
Threat Predictability x Congruence x Aggression & .01 & $(1,112)$ & .94 & .00 \\
Overall Threat x Congruence x Aggression & .01 & $(1,112)$ & .93 & .00
\end{tabular}

\footnotetext{
${ }^{7}$ Frontocentral and central P3 analyses revealed no significant effects, though there was a marginally significant Aggression $\mathrm{x}$ Threat Predictability effect at the central site, such that higher levels of aggression were related to less reduced central P3 during unpredictable vs. predictable threat contexts, see Appendix O for more information.
} 
Table 12. (Continued)

Parietal Site - P3

Threat Predictability

Overall Threat

Congruence

Aggression (Between Subjects)

Threat Predictability x Aggression

Overall Threat x Aggression

Congruence x Aggression

Threat Predictability $\mathrm{x}$ Congruence

Overall Threat $\mathrm{x}$ Congruence

Threat Predictability $\mathrm{x}$ Congruence $\mathrm{x}$ Aggression

Overall Threat $\mathrm{x}$ Congruence $\mathrm{x}$ Aggression

Note. ${ }^{*} p<.05,{ }^{* *} p<.01,{ }^{* * *} p<.001, \wedge$ marginal effect $(p>.05)$.

\begin{tabular}{llll}
.38 & $(1,112)$ & .56 & .00 \\
13.33 & $(1,112)$ & $.00^{* * *}$ & .12 \\
.22 & $(1,112)$ & .64 & .00 \\
.72 & $(1,112)$ & .40 & .01 \\
.19 & $(1,112)$ & .66 & .00 \\
4.76 & $(1,112)$ & $.03^{*}$ & .04 \\
1.35 & $(1,112)$ & .25 & .01 \\
.9 & $(1,112)$ & .35 & .01 \\
1.05 & $(1,112)$ & .31 & .01 \\
.89 & $(1,112)$ & .35 & .01 \\
3.23 & $(1,112)$ & $.08^{\wedge}$ & .03 \\
\hline
\end{tabular}

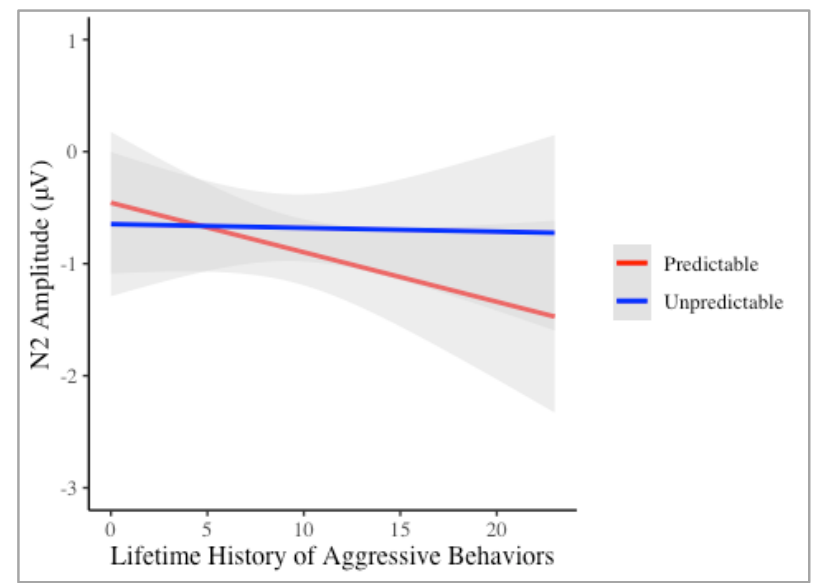

Figure 11. Aggression x Threat Predictability Interaction at Frontocentral N2.

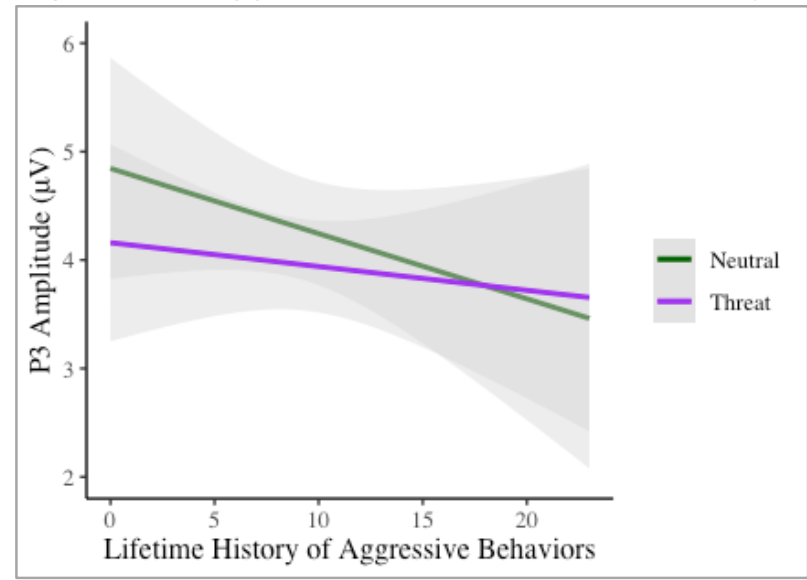

Figure 12. Aggression x Overall Threat Interaction at Parietal P3. 


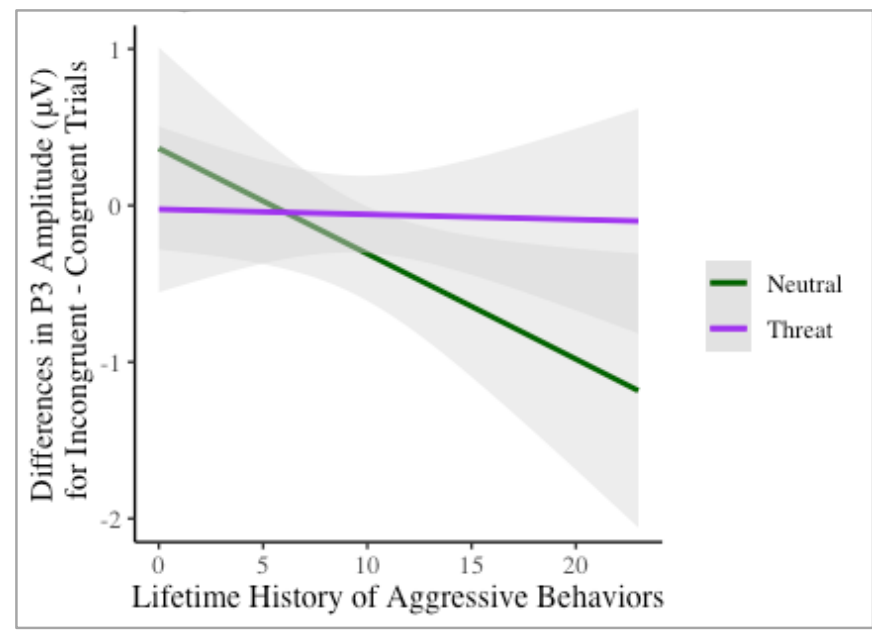

Figure 13. Aggression x Overall Threat x Congruence Interaction at Parietal P3.

For the behavioral measures, analyses of $\underline{R T}$ and accuracy revealed no effects involving aggressive behavior (see Table 13).

Table 13. GLM Effects of Behavior as a function of Aggressive Behavior.

\begin{tabular}{lllll}
\hline & $\boldsymbol{F}$ & $\mathbf{d f}$ & $\boldsymbol{p}$ & $\mathbf{\eta}_{\mathbf{p}}{ }^{2}$ \\
\hline Reaction Time & & & & \\
\hline Threat Predictability & .23 & $(1,136)$ & .63 & .00 \\
Overall Threat & .23 & $(1,136)$ & .63 & .00 \\
Congruence & 123.71 & $(1,136)$ & $.00 * *$ & .48 \\
Aggression (Between Subjects) & 1.53 & $(1,136)$ & .22 & .01 \\
Threat Predictability x Aggression & .01 & $(1,136)$ & .19 & .00 \\
Overall Threat x Aggression & .13 & $(1,136)$ & .93 & .00 \\
Congruence x Aggression & .01 & $(1,136)$ & .73 & .00 \\
Threat Predictability x Congruence & 1.03 & $(1,136)$ & .31 & .01 \\
Overall Threat x Congruence & .05 & $(1,136)$ & .82 & .00 \\
Threat Predictability x Congruence x Aggression & .63 & $(1,136)$ & .43 & .01 \\
Overall Threat x Congruence x Aggression & .60 & $(1,136)$ & .44 & .00 \\
\hline Accuracy & & & & \\
\hline Threat Predictability & 2.17 & $(1,136)$ & .14 & .02 \\
Overall Threat & .34 & $(1,136)$ & .56 & .00 \\
Congruence & 8.42 & $(1,136)$ & $.00 * *$ & .06 \\
Aggression (Between Subjects) & 1.16 & $(1,136)$ & .21 & .01 \\
Threat Predictability x Aggression & .03 & $(1,136)$ & .85 & .00 \\
Overall Threat x Aggression & .43 & $(1,136)$ & .51 & .00 \\
Congruence x Aggression & .00 & $(1,136)$ & .98 & .00 \\
Threat Predictability x Congruence & .19 & $(1,136)$ & .66 & .00 \\
Overall Threat x Congruence & .00 & $(1,136)$ & 1.00 & .00 \\
Threat Predictability x Congruence x Aggression & .57 & $(1,136)$ & .45 & .00 \\
Overall Threat x Congruence x Aggression & .37 & $(1,136)$ & .55 & .00 \\
\hline Note $p<.05, *<.01, * *$ \\
\hline
\end{tabular}

Note. ${ }^{*} p<.05, * * p<.01, * * * p<.001, \wedge$ marginal effect $(p>.05)$. 
Together these results suggest that higher levels of aggressive behavior were associated with decreased (less negative) N2 during unpredictable (vs. predictable) threat. Aggression also related to overall blunted (less positive) P3 across conditions, with surprisingly less blunting of the P3 and of congruence differentiation during conditions of overall threat relative to no threat conditions. Finally, we found no effect of aggression on behavioral indicators of task performance, namely accuracy and reaction time.

3.3.4. Self-Harm Results. Analyses of the $\underline{P 3}$ revealed no effects involving self-harm (combined suicidal and non-suicidal) behavior ${ }^{8}$, whereas analyses of the $\underline{N 2}$ revealed a small, marginally significant three-way Self-Harm x Overall Threat (P/U vs. N) x Congruence interaction, $F(1,112)=2.92, p=.09, \eta_{\mathrm{p}}{ }^{2}=.03$ (see Table 14). Follow up analyses revealed that the Self-Harm x Congruence interaction was not significant in either threat condition (Threat: $F(1,112)=0.02, p=.88, \eta_{\mathrm{p}}{ }^{2}=.00 ;$ No Threat: $\left.F(1,112)=0.02, p=.89, \eta_{\mathrm{p}}{ }^{2}=.00\right)$; however, self-harm was associated with marginally less N2 differentiation of incongruent and congruent flankers during no threat $(r=.13, p=.17)$ but not during threat $(r=-.03, p=.75)$. See Figure 14 .

Table 14. GLM Effects of ERPs as a function of Self-Harm Behavior.

\begin{tabular}{lllll}
\hline Frontocentral Site - N2 & $\boldsymbol{F}$ & $\mathbf{d f}$ & $\boldsymbol{p}$ & $\boldsymbol{\eta}_{\mathbf{p}}{ }^{2}$ \\
\hline Threat Predictability & & & & \\
Overall Threat & 2.69 & $(1,112)$ & .10 & .02 \\
Congruence & .51 & $(1,112)$ & .48 & .00 \\
Self-Harm (Between Subjects) & 13.75 & $(1,112)$ & $.00^{* * * *}$ & .11 \\
Threat Predictability x Self-Harm & .00 & $(1,112)$ & .96 & .00 \\
Overall Threat x Self-Harm & .00 & $(1,112)$ & .99 & .00 \\
Congruence x Self-Harm & .32 & $(1,112)$ & .57 & .00 \\
Threat Predictability x Congruence & .12 & $(1,112)$ & .73 & .00 \\
Overall Threat x Congruence & .00 & $(1,112)$ & .95 & .00 \\
Threat Predictability x Congruence x Self-Harm & 4.36 & $(1,112)$ & $.04^{*}$ & .04 \\
Overall Threat x Congruence x Self-Harm & 1.93 & $(1,112)$ & .17 & .02 \\
Parietal Site - P3 & 2.92 & $(1,112)$ & $.09^{\wedge}$ & .03 \\
\hline Threat Predictability & & & & \\
\hline
\end{tabular}

\footnotetext{
${ }^{8}$ Self-harm was also unrelated to P3 amplitude at frontocentral and central sites, refer to Appendix O.
} 
Table 14. (Continued)

\begin{tabular}{lllll} 
Overall Threat & 15.70 & $(1,112)$ & $.00^{* * *}$ & .12 \\
Congruence & 2.25 & $(1,112)$ & .14 & .02 \\
Self-Harm (Between Subjects) & .05 & $(1,112)$ & .82 & .00 \\
Threat Predictability x Self-Harm & .15 & $(1,112)$ & .70 & .00 \\
Overall Threat x Self-Harm & 2.59 & $(1,112)$ & .11 & .02 \\
Congruence x Self-Harm & .79 & $(1,112)$ & .38 & .01 \\
Threat Predictability x Congruence & .02 & $(1,112)$ & .90 & .00 \\
Overall Threat x Congruence & .34 & $(1,112)$ & .56 & .00 \\
Threat Predictability x Congruence x Self-Harm & .02 & $(1,112)$ & .89 & .00 \\
Overall Threat x Congruence x Self-Harm & .67 & $(1,112)$ & .42 & .01 \\
\hline
\end{tabular}

Note. ${ }^{*} p<.05,{ }^{* *} p<.01,{ }^{* * *} p<.001, \wedge$ marginal effect $(p>.05)$. Self-harm was log transformed.

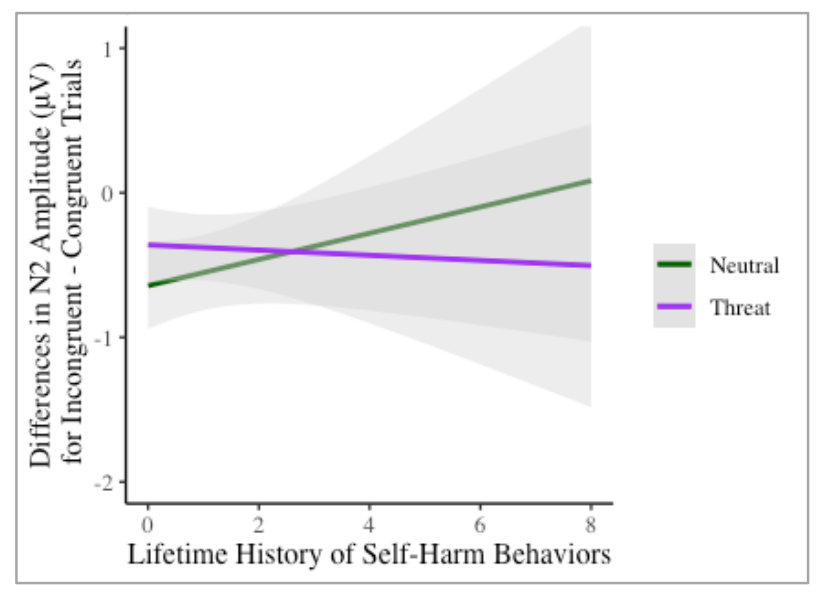

Figure 14. Self-Harm x Overall Threat x Congruence Interaction at Frontocentral N2.

For the behavioral measures, analyses of accuracy revealed no effects involving selfharm, whereas analyses of $\underline{R T}$ revealed only a marginally-significant two-way Self-harm $\mathrm{x}$ Congruence interaction, $F(1,136)=3.70, p=.06, \eta_{\mathrm{p}}{ }^{2}=.03$ (see Table 15). Self-harm was associated with slightly faster RT during incongruent $(r=-.14, p=.12)$ vs. congruent $(r=-.11, p$ $=.20$ ) trials, resulting in reduced flanker congruence at high levels of self-harm (see Figure 15).

Table 15. GLM Effects of Behavior as a function of Self-Harm Behavior.

\begin{tabular}{lllll}
\hline Reaction Time & $\boldsymbol{F}$ & $\mathbf{d f}$ & $\boldsymbol{p}$ & $\boldsymbol{\eta}_{\mathbf{p}}{ }^{2}$ \\
\hline Threat Predictability & & & & \\
Overall Threat & .31 & $(1,136)$ & .58 & .00 \\
Congruence & .54 & $(1,136)$ & .46 & .00 \\
Self-Harm (Between Subjects) & 475.31 & $(1,136)$ & $.00^{* * *}$ & .78 \\
Threat Predictability x Self-Harm & 1.90 & $(1,136)$ & .17 & .01 \\
Overall Threat x Self-Harm & .19 & $(1,136)$ & .67 & .00 \\
& .74 & $(1,136)$ & .39 & .01
\end{tabular}


Table 15. (Continued)

\begin{tabular}{lllll}
\hline Congruence x Self-Harm & 3.70 & $(1,136)$ & $.06^{\wedge}$ & .03 \\
Threat Predictability x Congruence & .05 & $(1,136)$ & .83 & .00 \\
Overall Threat x Congruence & .93 & $(1,136)$ & .34 & .01 \\
Threat Predictability x Congruence x Self-Harm & .53 & $(1,136)$ & .47 & .00 \\
Overall Threat x Congruence x Self-Harm & .06 & $(1,136)$ & .81 & .00 \\
\hline Accuracy & & & & \\
\hline Threat Predictability & 8.41 & $(1,136)$ & $.00^{* *}$ & .06 \\
Overall Threat & 4.03 & $(1,136)$ & $.05^{*}$ & .03 \\
Congruence & 28.59 & $(1,136)$ & $.00^{* * *}$ & .17 \\
Self-Harm (Between Subjects) & .45 & $(1,136)$ & .50 & .00 \\
Threat Predictability x Self-Harm & .95 & $(1,136)$ & .33 & .01 \\
Overall Threat x Self-Harm & .06 & $(1,136)$ & .81 & .00 \\
Congruence x Self-Harm & .01 & $(1,136)$ & .92 & .00 \\
Threat Predictability x Congruence & 3.31 & $(1,136)$ & $.07^{\wedge}$ & .02 \\
Overall Threat x Congruence & .14 & $(1,136)$ & .71 & .00 \\
Threat Predictability x Congruence x Self-Harm & .17 & $(1,136)$ & .68 & .00 \\
Overall Threat x Congruence x Self-Harm & 1.48 & $(1,136)$ & .23 & .01 \\
\hline
\end{tabular}

Note. ${ }^{*} p<.05,{ }^{* *} p<.01,{ }^{* * *} p<.001, \wedge$ marginal effect $(p>.05)$. Self-harm was log transformed.

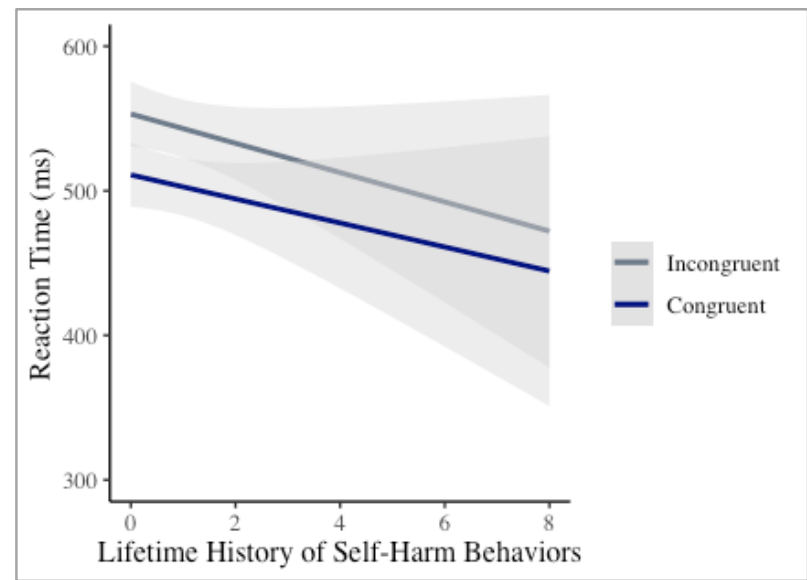

Figure 15. Self-Harm x Congruence Interaction of Reaction Time.

Together these results indicate high rates of lifetime self-harm behaviors (both suicidal and non-suicidal) were associated with somewhat increased N2 flanker congruence differentiation during threat conditions, and with faster reaction time to both flanker trial types, resulting in less congruence differentiation at high levels of self-harm. 
3.3.5. Alcohol Use Disorder Symptoms. Analyses of the $\underline{N 2}$ and $\underline{P 3}$ revealed no effects involving lifetime AUD symptoms (see Table 16) ${ }^{9}$.

Table 16. GLM Effects of ERPs as a function of AUD Symptoms.

\begin{tabular}{lllll}
\hline & $\boldsymbol{F}$ & $\mathbf{d f}$ & $\boldsymbol{p}$ & $\boldsymbol{\eta}_{\mathbf{p}}{ }^{2}$ \\
\hline Frontocentral Site - N2 & & & & \\
\hline Threat Predictability & 2.49 & $(1,112)$ & .12 & .02 \\
Overall Threat & 2.81 & $(1,112)$ & .10 & .03 \\
Congruence & 10.13 & $(1,112)$ & $.00^{* *}$ & .08 \\
AUD (Between Subjects) & .56 & $(1,112)$ & .49 & .01 \\
Threat Predictability x AUD & .10 & $(1,112)$ & .76 & .00 \\
Overall Threat x AUD & 1.34 & $(1,112)$ & .25 & .01 \\
Congruence x AUD & .16 & $(1,112)$ & .69 & .00 \\
Threat Predictability x Congruence & .47 & $(1,112)$ & .50 & .00 \\
Overall Threat x Congruence & .04 & $(1,112)$ & .85 & .00 \\
Threat Predictability x Congruence x AUD & .00 & $(1,112)$ & .98 & .00 \\
Overall Threat x Congruence x AUD & 2.52 & $(1,112)$ & .12 & .02 \\
\hline Parietal Site - P3 & & & & \\
\hline Threat Predictability & .92 & $(1,112)$ & .34 & .01 \\
Overall Threat & 7.90 & $(1,112)$ & $.01 * *$ & .07 \\
Congruence & 1.13 & $(1,112)$ & .29 & .01 \\
AUD (Between Subjects) & 1.73 & $(1,112)$ & .19 & .02 \\
Threat Predictability x AUD & .45 & $(1,112)$ & .51 & .00 \\
Overall Threat x AUD & .17 & $(1,112)$ & .68 & .00 \\
Congruence x AUD & .10 & $(1,112)$ & .75 & .00 \\
Threat Predictability x Congruence & .02 & $(1,112)$ & .88 & .00 \\
Overall Threat x Congruence & .08 & $(1,112)$ & .77 & .00 \\
Threat Predictability x Congruence x AUD & .19 & $(1,112)$ & .67 & .00 \\
Overall Threat x Congruence x AUD & 2.47 & $(1,112)$ & .12 & .02 \\
\hline
\end{tabular}

Note. ${ }^{*} p<.05,{ }^{* *} p<.01,{ }^{* * *} p<.001,{ }^{\wedge}$ marginal effect $(p>.05)$.

For the behavioral measures, analyses revealed only a small, between subjects effect of AUD symptoms on $\underline{R T}, F(1,136)=3.56, p=.06, \eta_{\mathrm{p}}^{2}=.03$, such that greater symptoms of AUD were associated with faster overall reaction times on the task, $r=-.16, p=.06$. There were no effects of $\underline{\text { accuracy }}$ involving AUD symptoms. See Table 17.

\footnotetext{
${ }^{9}$ AUD symptoms were also unrelated to P3 amplitude at frontocentral and central sites, refer to Appendix O.
} 
Table 17. GLM Effects of Behavior as a function of AUD Symptoms.

\begin{tabular}{lllll} 
& $\boldsymbol{F}$ & $\mathbf{d f}$ & $\boldsymbol{p}$ & $\mathbf{\eta}_{\mathbf{p}}{ }^{2}$ \\
\hline Reaction Time & & & & .00 \\
Threat Predictability & .35 & $(1,136)$ & .55 & .01 \\
Overall Threat & .70 & $(1,136)$ & .40 & .01 \\
Congruence & 285.16 & $(1,136)$ & $.00^{* * *}$ & .68 \\
AUD (Between Subjects) & 3.56 & $(1,136)$ & $.06^{\wedge}$ & .03 \\
Threat Predictability x AUD & .00 & $(1,136)$ & .98 & .00 \\
Overall Threat x AUD & .68 & $(1,136)$ & .41 & .01 \\
Congruence x AUD & .28 & $(1,136)$ & .60 & .00 \\
Threat Predictability x Congruence & .76 & $(1,136)$ & .38 & .01 \\
Overall Threat x Congruence & 1.53 & $(1,136)$ & .22 & .01 \\
Threat Predictability x Congruence x AUD & .33 & $(1,136)$ & .57 & .00 \\
Overall Threat x Congruence x AUD & .62 & $(1,136)$ & .43 & .01 \\
\hline Accuracy & & & & \\
\hline Threat Predictability & 6.76 & $(1,136)$ & $.01 *$ & .05 \\
Overall Threat & 4.13 & $(1,136)$ & $.04 *$ & .03 \\
Congruence & 26.78 & $(1,136)$ & $.00 * * *$ & .16 \\
AUD (Between Subjects) & .95 & $(1,136)$ & .33 & .01 \\
Threat Predictability x AUD & .85 & $(1,136)$ & .36 & .01 \\
Overall Threat x AUD & .21 & $(1,136)$ & .65 & .00 \\
Congruence x AUD & 1.57 & $(1,136)$ & .21 & .01 \\
Threat Predictability x Congruence & 1.17 & $(1,136)$ & .28 & .01 \\
Overall Threat x Congruence & .00 & $(1,136)$ & .95 & .00 \\
Threat Predictability x Congruence x AUD & .58 & $(1,136)$ & .45 & .00 \\
Overall Threat x Congruence x AUD & 1.00 & $(1,136)$ & .32 & .01 \\
\hline
\end{tabular}

Note. ${ }^{*} p<.05,{ }^{* *} p<.01,{ }^{* * *} p<.001, \wedge$ marginal effect $(p>.05)$.

3.3.6. Substance Use Disorder Symptoms. $\underline{N 2}$ analyses revealed a between subjects effect of SUD symptoms on overall N2 amplitude, $F(1,112)=4.32, p=.04, \eta_{\mathrm{p}}{ }^{2}=.04$, such that greater symptoms of SUD were associated with enhanced (more negative) N2 amplitude (regardless of threat condition or flanker trial type), $r=-.20, p=.03$ (see Table 18). Analyses of the $\underline{P 3}$ revealed a large, three-way SUD x Overall Threat (P/U vs. N) x Congruence interaction, $F(1,112)=8.98, p=.003, \eta_{\mathrm{p}}{ }^{2}=.07^{10}$. Follow up analyses showed that the SUD x Congruence interaction was only significant during threat, with higher levels of SUD symptoms associated with greater flanker congruence differentiation (incongruent - congruent difference score) for

\footnotetext{
${ }^{10}$ This effect was specific to the parietal P3 analyses. SUD symptoms were unrelated to P3 amplitude at frontocentral and central sites, refer to Appendix $\mathrm{O}$.
} 
threat, $\left(F(1,112)=5.27, p=.02, \eta_{\mathrm{p}}{ }^{2}=.05 ; r=.21, p=.02\right)$, but with less flanker congruence differentiation under no threat $\left(F(1,112)=2.39, p=.13, \eta_{\mathrm{p}}{ }^{2}=.02 ; r=-.15, p=.13\right)$. See Figure 16 . Table 18. GLM Effects of ERPs as a function of SUD Symptoms.

\begin{tabular}{lllll}
\hline & $\boldsymbol{F}$ & $\mathbf{d f}$ & $\boldsymbol{p}$ & $\boldsymbol{\eta}_{\mathbf{p}}{ }^{2}$ \\
\hline Frontocentral Site - N2 & & & & \\
\hline Threat Predictability & 1.83 & $(1,112)$ & .18 & .02 \\
Overall Threat & .00 & $(1,112)$ & .99 & .00 \\
Congruence & 7.88 & $(1,112)$ & $.01^{* *}$ & .07 \\
SUD (Between Subjects) & 4.32 & $(1,112)$ & $.04^{*}$ & .04 \\
Threat Predictability x SUD & .03 & $(1,112)$ & .87 & .00 \\
Overall Threat x SUD & 1.81 & $(1,112)$ & .18 & .02 \\
Congruence x SUD & .21 & $(1,112)$ & .65 & .00 \\
Threat Predictability x Congruence & .46 & $(1,112)$ & .50 & .00 \\
Overall Threat x Congruence & 3.20 & $(1,112)$ & $.08^{\wedge}$ & .03 \\
Threat Predictability x Congruence x SUD & .00 & $(1,112)$ & .96 & .00 \\
Overall Threat x Congruence x SUD & 1.32 & $(1,112)$ & .25 & .01 \\
\hline Parietal Site - P3 & & & & \\
\hline Threat Predictability & 1.10 & $(1,112)$ & .30 & .01 \\
Overall Threat & 12.74 & $(1,112)$ & $.00^{* *}$ & .10 \\
Congruence & 2.50 & $(1,112)$ & .12 & .02 \\
SUD (Between Subjects) & .27 & $(1,112)$ & .60 & .00 \\
Threat Predictability x SUD & .57 & $(1,112)$ & .45 & .01 \\
Overall Threat x SUD & 1.60 & $(1,112)$ & .21 & .01 \\
Congruence x SUD & 1.03 & $(1,112)$ & .31 & .01 \\
Threat Predictability x Congruence & 1.01 & $(1,112)$ & .32 & .01 \\
Overall Threat x Congruence & 1.13 & $(1,112)$ & .29 & .01 \\
Threat Predictability x Congruence x SUD & 1.51 & $(1,112)$ & .22 & .01 \\
Overall Threat x Congruence x SUD & 8.98 & $(1,112)$ & $.00^{* *}$ & .07 \\
\hline
\end{tabular}

Note. ${ }^{*} p<.05,{ }^{* *} p<.01,{ }^{* * *} p<.001,{ }^{\wedge}$ marginal effect $(p>.05)$.

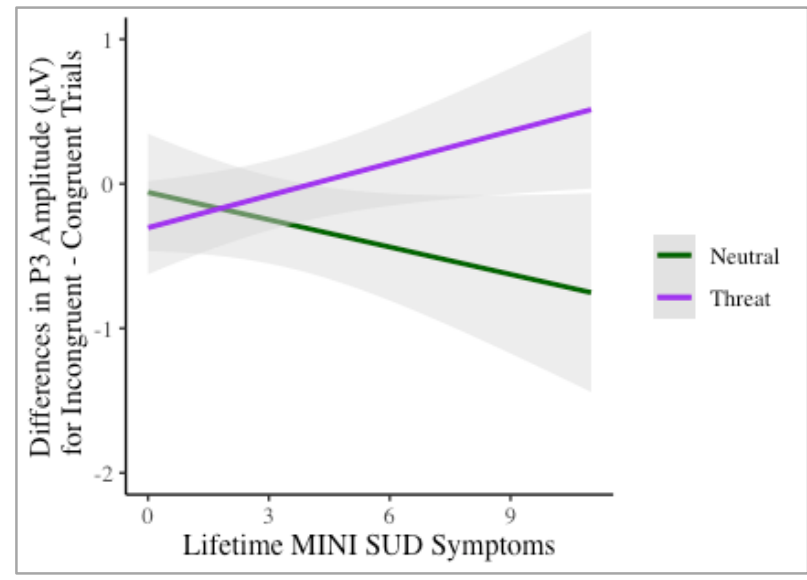

Figure 16. SUD x Overall Threat x Congruence Interaction at Parietal P3. 
For the behavioral measures, analyses of $\underline{R T}$ revealed both a two-way SUD x Congruence interaction, $F(136)=4.65, p=03, \eta_{\mathrm{p}}{ }^{2}=.03$ and a three-way SUD x Threat Predictability (P vs. $\mathrm{U}) \mathrm{x}$ Congruence interaction, $F(136)=4.81, p=.03, \eta_{\mathrm{p}}{ }^{2}=.03$, see Table 19. First, we decomposed the two-way SUD x Congruence interaction, which showed that SUD symptoms were associated with faster reaction time during both incongruent $(r=-.05, p=.53)$ and congruent ( $r=-.03, p=.75)$ trial types, at very small effect sizes (see Figure 17). The three-way SUD x Threat Predictability (P vs. U) x Congruence interaction instead indicated that the SUD $x$ Congruence interaction was only significant during the unpredictable threat $F(1,136)=6.41, p=$ $.01, \eta_{\mathrm{p}}{ }^{2}=.05$, with greater SUD symptoms related to decreased RT congruence differentiation (incongruent - congruent difference score) during unpredictable threat condition, $r=-.21, p=$ .01 but not during the predictable threat condition, $F(1,136)=0.16, p=.69, \eta_{\mathrm{p}}{ }^{2}=.00 ; r=-.04, p$ $=.69($ see Figure 18$)$.

Analyses of accuracy yielded a marginally significant two-way SUD x Overall threat $(\mathrm{P} / \mathrm{U}$ vs. $\mathrm{N})$ interaction, $F(1,136)=3.83, p=.05, \eta_{\mathrm{p}}{ }^{2}=.03$, which was superseded by a threeway SUD x Overall Threat (P/U vs. N) Congruence interaction, $F(1,136)=5.40, p=.02, \eta_{\mathrm{p}}{ }^{2}=$ .04 , see Table 19. We decomposed the three-way interaction to reveal that the effect of congruence was not significant in either threat condition (Threat: $F(1,136)=1.09, p=.30, \eta_{\mathrm{p}}{ }^{2}=$ .01 ; no threat: $\left.F(1,136)=1.29, p=.26, \eta_{\mathrm{p}}{ }^{2}=.01\right)$; instead, SUD symptoms were associated with increased accuracy on incongruent (vs. congruent) flankers during conditions of threat $(r=.09, p$ $=.30)$ and somewhat decreased accuracy during conditions of no threat $(r=-.10, p=.26)$. See Figure 19. 
Table 19. GLM Effects of Behavior as a function of SUD Symptoms.

\begin{tabular}{lllll} 
& $\boldsymbol{F}$ & $\mathbf{d f}$ & $\boldsymbol{p}$ & $\mathbf{\eta}_{\mathbf{p}}{ }^{2}$ \\
\hline Reaction Time & & & & \\
\hline Threat Predictability & 1.11 & $(1,136)$ & .29 & .01 \\
Overall Threat & .44 & $(1,136)$ & .51 & .00 \\
Congruence & 375.94 & $(1,136)$ & $.00^{* * *}$ & .73 \\
SUD (Between Subjects) & .13 & $(1,136)$ & .72 & .00 \\
Threat Predictability x SUD & .35 & $(1,136)$ & .56 & .00 \\
Overall Threat x SUD & .36 & $(1,136)$ & .55 & .00 \\
Congruence x SUD & 4.65 & $(1,136)$ & $.03^{*}$ & .03 \\
Threat Predictability x Congruence & 3.87 & $(1,136)$ & .05 & .03 \\
Overall Threat x Congruence & 1.36 & $(1,136)$ & .25 & .01 \\
Threat Predictability x Congruence x SUD & 4.81 & $(1,136)$ & $.03^{*}$ & .03 \\
Overall Threat x Congruence x SUD & .45 & $(1,136)$ & .50 & .00 \\
\hline Accuracy & & & & \\
\hline Threat Predictability & 3.8 & $(1,136)$ & $.05^{\wedge}$ & .03 \\
Overall Threat & .30 & $(1,136)$ & .59 & .00 \\
Congruence & 23.30 & $(1,136)$ & $.00^{* * *}$ & .15 \\
SUD (Between Subjects) & 1.99 & $(1,136)$ & .16 & .01 \\
Threat Predictability x SUD & .03 & $(1,136)$ & .86 & .00 \\
Overall Threat x SUD & 3.83 & $(1,136)$ & $.05^{\wedge}$ & .03 \\
Congruence x SUD & .17 & $(1,136)$ & .68 & .00 \\
Threat Predictability x Congruence & .43 & $(1,136)$ & .51 & .00 \\
Overall Threat x Congruence & .49 & $(1,136)$ & .49 & .00 \\
Threat Predictability x Congruence x SUD & 2.70 & $(1,136)$ & .10 & .02 \\
Overall Threat x Congruence x SUD & 5.40 & $(1,136)$ & $.02^{*}$ & .04 \\
\hline Note $p<.05, *<1$, & & &
\end{tabular}

Note. ${ }^{*} p<.05,{ }^{* *} p<.01,{ }^{* * *} p<.001, \wedge$ marginal effect $(p>.05)$

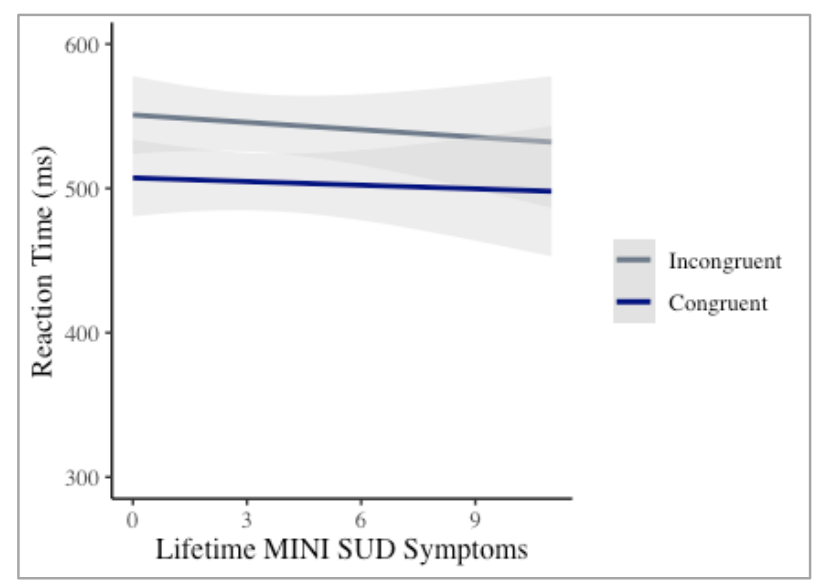

Figure 17. SUD x Congruence Interaction of Reaction Time. 


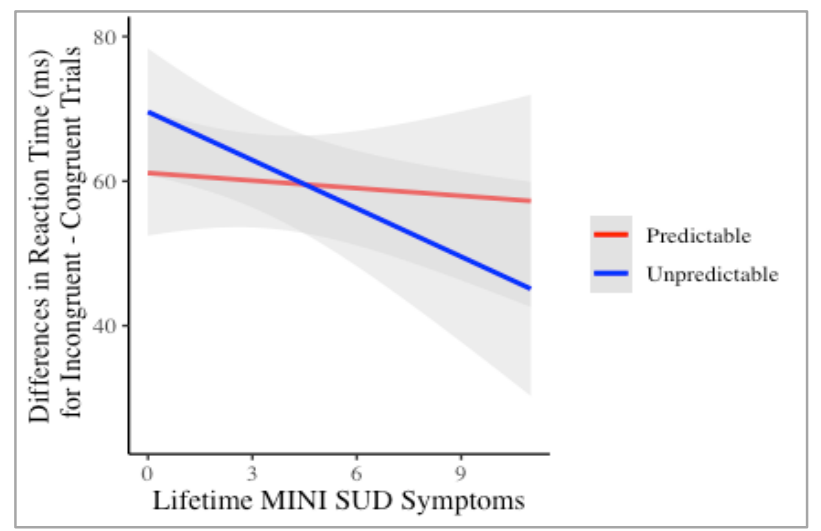

Figure 18. SUD x Threat Predictability x Congruence Interaction of Reaction Time.

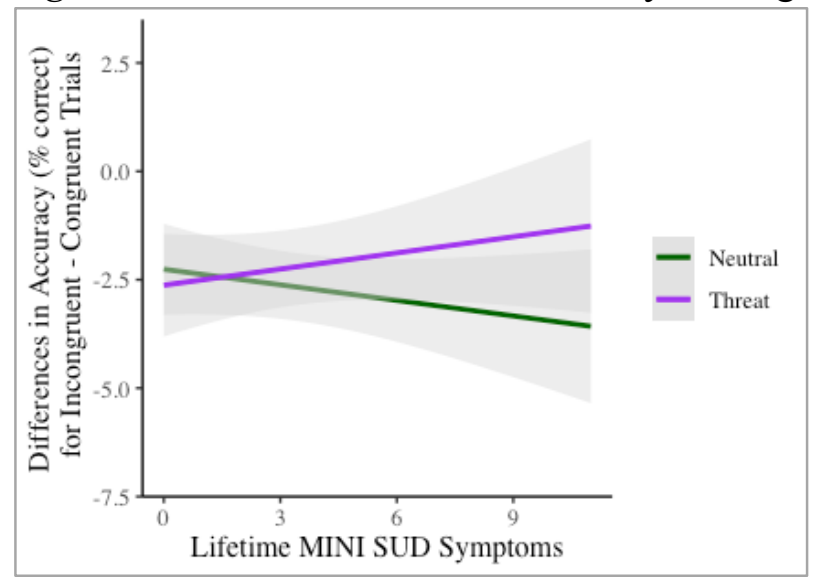

Figure 19. SUD x Overall Threat x Congruence Interaction of Accuracy.

In summary, individuals with higher levels of SUD symptoms had a heightened

(more negative) N2, and enhanced conflict processing (larger, more positive P3 for incongruent relative to congruent flankers) during conditions of threat relative to no threat. In terms of performance, they displayed less RT differentiation of incongruent versus congruent flanker trial types during unpredictable relative to predictable threat. Finally, greater SUD symptoms were associated with slightly less pronounced decreases in accuracy during conditions of threat and more accuracy differentiation during threat relative to no threat. 
Table 20. Summary of Study Effects.

\begin{tabular}{|c|c|c|c|}
\hline & DV & Effect & Interpretation \\
\hline \multirow{9}{*}{ Aim 1} & \multirow{2}{*}{$\mathrm{N} 2$} & (un)Predictability & $\mathrm{N} 2$ enhanced during $\mathrm{P}$ threat \\
\hline & & Congruence & N2 enhanced for incongruent flankers \\
\hline & \multirow{2}{*}{ P3 } & (un)Predictability & P3 especially blunted during $\mathrm{P}$ threat \\
\hline & & Overall Threat & P3 blunted during threat \\
\hline & RT & Congruence & RT slower on incongruent flankers \\
\hline & \multirow{4}{*}{ Accuracy } & (un)Predictability & $\begin{array}{l}\text { Accuracy especially improved during } \mathrm{P} \\
\text { threat }\end{array}$ \\
\hline & & Overall Threat & Accuracy improved during threat \\
\hline & & Congruence & $\begin{array}{l}\text { Accuracy decreased on incongruent } \\
\text { flankers }\end{array}$ \\
\hline & & $\begin{array}{l}\text { (un)Predictability } \mathrm{x} \\
\text { Congruence }\end{array}$ & $\begin{array}{l}\text { Accuracy decrements due to flanker } \\
\text { congruence interference (incongruent - } \\
\text { congruent difference) reduced during P } \\
\text { vs. U threat }\end{array}$ \\
\hline \multirow{3}{*}{$\begin{array}{l}\text { Disinhibition } \\
\text { Factor (DIS) }\end{array}$} & \multirow[b]{2}{*}{ P3 } & Dis x Overall Threat & $\begin{array}{l}\text { DIS related to enhanced P3 during } \\
\text { threat }\end{array}$ \\
\hline & & $\begin{array}{l}\text { Dis x Overall Threat } \\
\text { x Congruence }\end{array}$ & $\begin{array}{l}\text { DIS related to greater P3 flanker } \\
\text { congruence differentiation during threat } \\
\text { and less during no threat }\end{array}$ \\
\hline & Accuracy & $\begin{array}{l}\text { Dis x Overall Threat } \\
\text { x Congruence }\end{array}$ & $\begin{array}{l}\text { DIS related to fewer decrements in } \\
\text { accuracy due to flanker congruence } \\
\text { interference during threat }\end{array}$ \\
\hline \multirow{3}{*}{$\begin{array}{l}\text { Negative } \\
\text { Urgency } \\
\text { (NU) }\end{array}$} & \multirow{2}{*}{$\mathrm{N} 2$} & NU x Overall Threat & $\begin{array}{l}\text { NU related to enhanced N2 during } \\
\text { threat/ blunted N2 during no threat }\end{array}$ \\
\hline & & NU x Congruence & $\begin{array}{l}\text { NU related to blunted N2 to } \\
\text { incongruent flankers but enhanced N2 } \\
\text { to congruent flankers }\end{array}$ \\
\hline & Accuracy & NU x Overall Threat & $\begin{array}{l}\text { NU related to decreased accuracy } \\
\text { during no threat (unrelated during } \\
\text { threat) }\end{array}$ \\
\hline \multirow{3}{*}{$\begin{array}{l}\text { Aggression } \\
\text { (AGG) }\end{array}$} & $\mathrm{N} 2$ & $\begin{array}{l}\text { AGG x } \\
\text { (un)Predictability }\end{array}$ & $\begin{array}{l}\text { AGG related to enhanced } \mathrm{N} 2 \text { during } \mathrm{P} \\
\text { threat but unrelated to } \mathrm{N} 2 \text { during } \mathrm{U} \\
\text { threat }\end{array}$ \\
\hline & $\mathrm{P} 3$ & $\begin{array}{l}\text { AGG x Overall } \\
\text { Threat }\end{array}$ & $\begin{array}{l}\text { AGG related to generally blunted P3 } \\
\text { but less so during threat vs. no threat }\end{array}$ \\
\hline & P3 & $\begin{array}{l}\text { AGG x Overall } \\
\text { Threat x Congruence }\end{array}$ & $\begin{array}{l}\text { AGG related to less P3 flanker } \\
\text { congruence differentiation during no } \\
\text { threat (unrelated to P3 differentiation } \\
\text { during threat) }\end{array}$ \\
\hline Self-Harm & $\mathrm{N} 2$ & $\begin{array}{l}\text { Self-Harm x Overall } \\
\text { Threat x Congruence }\end{array}$ & $\begin{array}{l}\text { Self-harm related to less N2 flanker } \\
\text { congruence differentiation during no } \\
\text { threat (unrelated to flanker congruence } \\
\text { differentiation during threat) }\end{array}$ \\
\hline
\end{tabular}


Table 20. (Continued)

\begin{tabular}{|c|l|l|l|}
\hline & RT & $\begin{array}{l}\text { Self-Harm } x \\
\text { Congruence }\end{array}$ & $\begin{array}{l}\text { Self-harm related to faster RT on } \\
\text { incongruent flankers }\end{array}$ \\
\hline AUD & RT & Btw-SS & AUD related to faster RT overall \\
\hline \multirow{4}{*}{ SUD } & N2 & Btw-SS & SUD related to enhanced N2 overall \\
\cline { 2 - 4 } & \multirow{2}{*}{ P3 } & $\begin{array}{l}\text { SUD } x \text { Overall } \\
\text { Threat x Congruence }\end{array}$ & $\begin{array}{l}\text { SUD related to greater P3 flanker } \\
\text { congruence differentiation during threat } \\
\text { (non-significantly related to less flanker } \\
\text { differentiation during no threat) }\end{array}$ \\
\cline { 2 - 4 } & \multirow{2}{*}{ Accuracy } & SUD $x$ Cong & $\begin{array}{l}\text { SUD related to faster RT on } \\
\text { incongruent flankers }\end{array}$ \\
\cline { 2 - 4 } & $\begin{array}{l}\text { SUD } x \\
\text { (un)Predictability } x \\
\text { Congruence }\end{array}$ & $\begin{array}{l}\text { SUD related to faster RT on } \\
\text { incongruent flankers especially during } \\
\text { U threat (unrelated during P threat) }\end{array}$ \\
\cline { 2 - 4 } & & $\begin{array}{l}\text { SUD } x \text { Overall } \\
\text { Threat }\end{array}$ & $\begin{array}{l}\text { SUD related to fewer decrements in } \\
\text { accuracy during threat }\end{array}$ \\
\cline { 2 - 4 } & $\begin{array}{l}\text { SUD } x \text { Overall } \\
\text { Threat x Congruence }\end{array}$ & $\begin{array}{l}\text { SUD related to increased accuracy to } \\
\text { incongruent flankers during threat but } \\
\text { decreased during no threat }\end{array}$ \\
\hline
\end{tabular}

\subsection{Supplemental Analyses}

3.4.1. Accounting for Potential Confounds and Covariates. Several analyses were conducted in order to account for potential third variables in our study. First, in order to account for the impact of potential order effects, we included block order as a covariate across all study aims. The inclusion of block order as a covariate did not alter the size or direction of most of our findings (see Table 21). Adding block order as a covariate reduced the Aim 1 N2 Threat (un)predictability effect to non-significance, although the size of the effect was unaffected ( $p=$ $.05, \eta_{\mathrm{p}}{ }^{2}=.03$ reduced to $p=.06, \eta_{\mathrm{p}}{ }^{2}=.03$ ). Additionally, the SUD x Overall Threat interaction on accuracy was also reduced to non-significance, although again the effect size remained largely similar in size $\left(p=.02, \eta_{\mathrm{p}}{ }^{2}=.04\right.$ reduced to $\left.p=.05, \eta_{\mathrm{p}}{ }^{2}=.03\right)$.

In order to examine the impact of demographic variables relevant to cognitive control on threat-induced alterations in cognition, we examined zero-order relationships between our dependent measures and sex and age. Results revealed no significant associations between our 
dependent variables and sex; however, age was negatively associated with parietal P3 amplitude $(r=-.25)$. Thus, we added age as a covariate across our Aim 1 analyses, see Table 21. Findings revealed that including age into our Aim 1 models reduced most of the effects to nonsignificance, with the exception of the effect of overall threat on P3 amplitude which actually had a somewhat larger effect after accounting for age. However, age did not alter the strength or direction of our Aim 2 effects, with the exception of the Disinhibition x Overall Threat interaction at parietal P3 $\left(p=.04, \eta_{\mathrm{p}}{ }^{2}=.04\right.$ reduced to $\left.p=.07, \eta_{\mathrm{p}}{ }^{2}=.03\right)$ and the SUD x Overall Threat interaction of accuracy $\left(p=.02 \eta_{\mathrm{p}}{ }^{2}=.04\right.$ reduced to $\left.p=.08, \eta_{\mathrm{p}}{ }^{2}=.02\right)$, which were both reduced to non-significance but remained similar in effect size. Together these results suggest that age may be a potential confound for our Aim 1 effects; however, age did not seem to account for Aim 2 effects.

Next, because there were individual differences in the shock intensity that participants rated as "uncomfortable," and used as the threat stimulus during the experiment, shock intensity (in $\mathrm{mA}$ ) was added as a covariate in all Aim 2 analyses (see Table 21). Adding shock intensity as a covariate generally did not alter the size of effects. Of note, the Disinhibition x Overall Threat interaction on the parietal P3 no longer reached significance after including shock intensity into the model; however, the size of the effect was unaffected $\left(p=.04, \eta_{\mathrm{p}}{ }^{2}=.04\right.$ reduced to $p=.05$, $\left.\eta_{\mathrm{p}}^{2}=.04\right)$.

Finally, in order to examine whether relationships between disinhibited behaviors and threat-induced alterations in cognition could be accounted for by anxiety (since anxiety and disinhibition often co-occur; Grant et al., 2004), we included the PSWQ and the MASQAnxious Arousal (MASQ-AA) scale as covariates in Aim 2 analyses. See Table 21 for a summary of our effects, with and without adding physiological (measured with the MASQ-AA) 
and cognitive (measured with the PSWQ) symptoms of anxiety in the model. Results suggest that adding measures of anxiety into our model altered the strength of some of our effects, consistent with an interpretation that the relationships between disinhibition and threat-induced alterations in cognition can be partially accounted for by anxiety. However, since many of our effects remained similar in effect size (i.e. $<.01$ reduction in $\eta_{\mathrm{p}}{ }^{2}$ ) and interpretation, these results collectively suggest that our findings were not solely accounted for by anxiety. 
Table 21. Accounting for Potential Covariates.

\begin{tabular}{|c|c|c|c|c|c|c|c|c|}
\hline Measure & DV & Effect & $\begin{array}{l}\text { Without } \\
\text { covariates }\end{array}$ & $\begin{array}{c}\text { With } \\
\text { Block } \\
\text { Order } \\
\end{array}$ & $\begin{array}{l}\text { With } \\
\text { Age }\end{array}$ & $\begin{array}{c}\text { With } \\
\text { Shock } \\
\text { Intensity }\end{array}$ & $\begin{array}{c}\text { With } \\
\text { MASQ- } \\
\text { AA } \\
\end{array}$ & $\begin{array}{c}\text { With } \\
\text { PSWQ }\end{array}$ \\
\hline \multirow{9}{*}{ Aim 1} & \multirow{2}{*}{ N2 } & (un)Predictability & $\begin{array}{l}p=.05 \\
\eta_{\mathrm{p}}^{2}=.03\end{array}$ & $\begin{array}{l}p=.06 \\
\eta_{p}^{2}=.03\end{array}$ & $\begin{array}{r}* p=.30 \\
\eta_{p}^{2}=.01\end{array}$ & --- & --- & --- \\
\hline & & Congruence & $\begin{array}{l}p=.00 \\
\eta_{\mathrm{p}}{ }^{2}=.14\end{array}$ & $\begin{array}{l}p=.00 \\
\eta_{\mathrm{p}}{ }^{2}=.13\end{array}$ & $\begin{array}{r}* p=.71 \\
\eta_{p}^{2}=.00\end{array}$ & --- & --- & --- \\
\hline & \multirow{2}{*}{$\mathrm{P} 3$} & (un)Predictability & $\begin{array}{l}p=.04 \\
\eta_{\mathrm{p}}^{2}=.08\end{array}$ & $\begin{aligned} *_{p} & =.03 \\
\eta_{\mathrm{p}} & =.04\end{aligned}$ & $\begin{array}{r}* p=.63 \\
\eta_{p}^{2}=.00\end{array}$ & --- & --- & --- \\
\hline & & Overall Threat & $\begin{array}{l}p=.00 \\
\eta_{\mathrm{p}}^{2}=.06\end{array}$ & $\begin{array}{l}\wedge_{p}=.00 \\
\eta_{\mathrm{p}}{ }^{2}=.10\end{array}$ & $\begin{array}{l}\wedge p=.00 \\
\eta_{\mathrm{p}}{ }^{2}=.09\end{array}$ & --- & --- & --- \\
\hline & RT & Congruence & $\begin{array}{l}p=.00 \\
\eta_{\mathrm{p}}{ }^{2}=.81\end{array}$ & $\begin{array}{l}p=.00 \\
\eta_{\mathrm{p}}{ }^{2}=.81\end{array}$ & $\begin{array}{r}* p=.00 \\
\eta_{\mathrm{p}}{ }^{2}=.08\end{array}$ & --- & --- & --- \\
\hline & \multirow{4}{*}{ Accuracy } & (un)Predictability & $\begin{array}{l}p=.01 \\
\eta_{\mathrm{p}}^{2}=.05\end{array}$ & $\begin{array}{l}p=.01 \\
\eta_{\mathrm{p}}{ }^{2}=.05\end{array}$ & $\begin{array}{r}* p=.08 \\
\eta_{p}^{2}=.02\end{array}$ & --- & --- & --- \\
\hline & & Overall Threat & $\begin{array}{l}p=.02 \\
\eta_{\mathrm{p}}^{2}=.04\end{array}$ & $\begin{array}{l}p=.02 \\
\eta_{\mathrm{p}}^{2}=.04\end{array}$ & $\begin{array}{r}* p=.81 \\
\eta_{p}^{2}=.00\end{array}$ & --- & --- & --- \\
\hline & & Congruence & $\begin{array}{l}p=.00 \\
\eta_{\mathrm{p}}^{2}=.22\end{array}$ & $\begin{array}{l}p=.00 \\
\eta_{\mathrm{p}}^{2}=.21\end{array}$ & $\begin{array}{l}{ }^{*} p=.63 \\
\eta_{p}^{2}=.00\end{array}$ & --- & --- & --- \\
\hline & & $\begin{array}{l}\text { (un)Predictability x } \\
\text { Congruence }\end{array}$ & $\begin{array}{l}p=.02 \\
\eta_{p}^{2}=.04\end{array}$ & $\begin{array}{l}p=.02 \\
\eta_{p}^{2}=.04\end{array}$ & $\begin{array}{r}* p=.15 \\
\eta_{p}^{2}=.02\end{array}$ & --- & --- & --- \\
\hline \multirow{3}{*}{ Disinhibition } & \multirow{2}{*}{$\mathrm{P} 3$} & Dis x Overall Threat & $\begin{array}{l}p=.04, \eta_{\mathrm{p}}^{2} \\
=.04\end{array}$ & $\begin{array}{l}p<.05 \\
\eta_{\mathrm{p}}{ }^{2}=.04\end{array}$ & $\begin{array}{l}p=.07 \\
\eta_{p}^{2}=.03\end{array}$ & $\begin{array}{l}p=.05 \\
\eta_{p}^{2}=.04\end{array}$ & $\begin{array}{l}p=.02 \\
\eta_{\mathrm{p}}{ }^{2}=.05\end{array}$ & $\begin{array}{l}p=.03 \\
\eta_{\mathrm{p}}{ }^{2}=.04\end{array}$ \\
\hline & & $\begin{array}{l}\text { Dis x Overall Threat } \mathrm{x} \\
\text { Congruence }\end{array}$ & $\begin{array}{l}p=.03, \eta_{\mathrm{p}}^{2} \\
=.05\end{array}$ & $\begin{array}{l}p=.03 \\
\eta_{\mathrm{p}}{ }^{2}=.04\end{array}$ & $\begin{array}{l}p=.02 \\
\eta_{\mathrm{p}}^{2}=.05\end{array}$ & $\begin{array}{l}p=.03 \\
\eta_{\mathrm{p}}^{2}=.04\end{array}$ & $\begin{array}{l}p=.02 \\
\eta_{\mathrm{p}}{ }^{2}=.05\end{array}$ & $\begin{array}{l}p=.02 \\
\eta_{\mathrm{p}}^{2}=.05\end{array}$ \\
\hline & Accuracy & $\begin{array}{l}\text { Dis x Overall Threat } \mathrm{x} \\
\text { Congruence }\end{array}$ & $\begin{array}{l}p<.05, \eta_{\mathrm{p}}^{2} \\
=.03\end{array}$ & $\begin{array}{l}p=.05 \\
\eta_{\mathrm{p}}^{2}=.03\end{array}$ & $\begin{array}{l}p=.04 \\
\eta_{\mathrm{p}}{ }^{2}=.03\end{array}$ & $\begin{array}{l}p=.04 \\
\eta_{\mathrm{p}}^{2}=.04\end{array}$ & $\begin{array}{l}p=.09 \\
\eta_{p}^{2}=.02\end{array}$ & $\begin{array}{l}p=.06 \\
\eta_{p}^{2}=.03\end{array}$ \\
\hline \multirow[b]{2}{*}{$\begin{array}{l}\text { Negative } \\
\text { Urgency }\end{array}$} & \multirow[b]{2}{*}{$\mathrm{N} 2$} & NU x Overall Threat & $\begin{array}{l}p=.09 \\
\eta_{\mathrm{p}}^{2}=.03\end{array}$ & $\begin{array}{l}p=.09 \\
\eta_{p}{ }^{2}=.03\end{array}$ & $\begin{array}{l}p=.09 \\
\eta_{\mathrm{p}}{ }^{2}=.03\end{array}$ & $\begin{array}{l}p=.09 \\
\eta_{\mathrm{p}}^{2}=.03\end{array}$ & $\begin{array}{l}p=.18 \\
\eta_{\mathrm{p}}^{2}=.02\end{array}$ & $\begin{array}{l}p=.17 \\
\eta_{\mathrm{p}}^{2}=.02\end{array}$ \\
\hline & & NU x Congruence & $\begin{array}{l}p=.02, \eta_{\mathrm{p}}^{2} \\
=.05\end{array}$ & $\begin{array}{l}p=.02 \\
\eta_{\mathrm{p}}{ }^{2}=.05\end{array}$ & $\begin{array}{l}p=.02 \\
\eta_{\mathrm{p}}{ }^{2}=.05\end{array}$ & $\begin{array}{l}p=.02 \\
\eta_{\mathrm{p}}{ }^{2}=.05\end{array}$ & $\begin{array}{l}p=.02 \\
\eta_{\mathrm{p}}{ }^{2}=.05\end{array}$ & $\begin{array}{c}{ }^{*} p=.16 \\
\eta_{p}{ }^{2}=.02\end{array}$ \\
\hline
\end{tabular}


Table 21. (Continued)

\begin{tabular}{|c|c|c|c|c|c|c|c|c|}
\hline & Accuracy & NU x Overall Threat & $\begin{array}{l}p=.09 \\
\eta_{\mathrm{p}}{ }^{2}=.02\end{array}$ & $\begin{array}{l}p=.10 \\
\eta_{\mathrm{p}}^{2}=.02\end{array}$ & $\begin{array}{l}p=.21 \\
\eta_{\mathrm{p}}^{2}=.01\end{array}$ & $\begin{array}{l}p=.10 \\
\eta_{\mathrm{p}}{ }^{2}=.02\end{array}$ & $\begin{array}{l}p=.12 \\
\eta_{\mathrm{p}}^{2}=.02\end{array}$ & $\begin{array}{l}p=.18 \\
\eta_{\mathrm{p}}^{2}=.02\end{array}$ \\
\hline \multirow{3}{*}{ Aggression } & N2 & AGG x (un)Predictability & $\begin{array}{l}p=.08 \\
\eta_{\mathrm{p}}^{2}=.03\end{array}$ & $\begin{array}{l}p=.06 \\
\eta_{\mathrm{p}}^{2}=.03\end{array}$ & $\begin{array}{l}p=.05 \\
\eta_{\mathrm{p}}^{2}=.04\end{array}$ & $\begin{array}{l}p=.06 \\
\eta_{\mathrm{p}}^{2}=.03\end{array}$ & $\begin{array}{l}p=.11 \\
\eta_{\mathrm{p}}^{2}=.02\end{array}$ & $\begin{array}{l}p=.04 \\
\eta_{\mathrm{p}}^{2}=.04\end{array}$ \\
\hline & P3 & AGG x Overall Threat & $\begin{array}{l}p=.03, \eta_{\mathrm{p}}^{2} \\
=.04\end{array}$ & $\begin{array}{l}p=.03 \\
\eta_{\mathrm{p}}^{2}=.04\end{array}$ & $\begin{array}{l}p=.04 \\
\eta_{\mathrm{p}}^{2}=.04\end{array}$ & $\begin{array}{l}p<.05 \\
\eta_{\mathrm{p}}^{2}=.04\end{array}$ & $\begin{array}{l}*{ }^{*} p=.12 \\
\eta_{p}^{2}=.02\end{array}$ & $\begin{array}{r}* p=.16 \\
\eta_{p}{ }^{2}=.02\end{array}$ \\
\hline & P3 & $\begin{array}{l}\text { AGG x Overall Threat } \mathrm{x} \\
\text { Congruence }\end{array}$ & $\begin{array}{l}p=.08, \eta_{\mathrm{p}}^{2} \\
=.03\end{array}$ & $\begin{array}{l}p=.07 \\
\eta_{\mathrm{p}}{ }^{2}=.03\end{array}$ & $\begin{array}{l}p=.06 \\
\eta_{p}{ }^{2}=.03\end{array}$ & $\begin{array}{l}p=.09 \\
\eta_{\mathrm{p}}{ }^{2}=.03\end{array}$ & $\begin{array}{l}p=.12 \\
\eta_{\mathrm{p}}^{2}=.02\end{array}$ & $\begin{array}{l}p=.16 \\
\eta_{p}^{2}=.02\end{array}$ \\
\hline \multirow{2}{*}{ Self-Harm } & $\mathrm{N} 2$ & $\begin{array}{l}\text { Self-Harm x Overall } \\
\text { Threat x Congruence }\end{array}$ & $\begin{array}{l}p=.09 \\
\eta_{\mathrm{p}}^{2}=.03\end{array}$ & $\begin{array}{l}p=.10 \\
\eta_{\mathrm{p}}^{2}=.02\end{array}$ & $\begin{array}{l}p=.08 \\
\eta_{\mathrm{p}}^{2}=.03\end{array}$ & $\begin{array}{l}p=.09 \\
\eta_{\mathrm{p}}^{2}=.03\end{array}$ & $\begin{array}{l}p=.12 \\
\eta_{\mathrm{p}}^{2}=.02\end{array}$ & $\begin{array}{l}p=.08 \\
\eta_{\mathrm{p}}^{2}=.03\end{array}$ \\
\hline & $\mathrm{RT}$ & Self-Harm x Congruence & $\begin{array}{l}p=.06 \\
\eta_{\mathrm{p}}^{2}=.03\end{array}$ & $\begin{array}{l}p=.06 \\
\eta_{\mathrm{p}}^{2}=.03\end{array}$ & $\begin{array}{l}p=.06 \\
\eta_{\mathrm{p}}^{2}=.03\end{array}$ & $\begin{array}{l}p=.05 \\
\eta_{\mathrm{p}}^{2}=.03\end{array}$ & $\begin{array}{l}p=.11 \\
\eta_{\mathrm{p}}^{2}=.02\end{array}$ & $\begin{array}{l}p=.09 \\
\eta_{\mathrm{p}}^{2}=.02\end{array}$ \\
\hline AUD & RT & Btw-SS & $\begin{array}{l}p=.06 \\
\eta_{p}^{2}=.03\end{array}$ & $\begin{array}{l}p=.05 \\
\eta_{p}^{2}=.03\end{array}$ & $\begin{array}{l}p=.04 \\
\eta_{p}^{2}=.03\end{array}$ & $\begin{array}{l}p=.10 \\
\eta_{p}^{2}=.02\end{array}$ & $\begin{array}{l}p=.03 \\
\eta_{p}^{2}=.04\end{array}$ & $\begin{array}{l}p=.05 \\
\eta_{\mathrm{p}}^{2}=.03\end{array}$ \\
\hline \multirow{6}{*}{ SUD } & N2 & Btw-SS & $\begin{array}{l}p=.04 \\
\eta_{\mathrm{p}}^{2}=.04\end{array}$ & $\begin{array}{l}p=.04 \\
\eta_{p}^{2}=.04\end{array}$ & $\begin{array}{l}p=.04 \\
\eta_{\mathrm{p}}^{2}=.04\end{array}$ & $\begin{array}{l}p=.04 \\
\eta_{p}^{2}=.04\end{array}$ & $\begin{array}{l}p=.09 \\
\eta_{p}^{2}=.03\end{array}$ & $\begin{array}{l}p=.05 \\
\eta_{p}^{2}=.04\end{array}$ \\
\hline & P3 & $\begin{array}{l}\text { SUD x Overall Threat } \mathrm{x} \\
\text { Congruence }\end{array}$ & $\begin{array}{l}p=.00 \\
\eta_{\mathrm{p}}^{2}=.07\end{array}$ & $\begin{array}{l}p=.00 \\
\eta_{p}^{2}=.08\end{array}$ & $\begin{array}{l}p=.00 \\
\eta_{p}^{2}=.08\end{array}$ & $\begin{array}{l}p=.00 \\
\eta_{\mathrm{p}}^{2}=.07\end{array}$ & $\begin{array}{c}*_{p}=.02 \\
\eta_{\mathrm{p}}^{2}=.05\end{array}$ & $\begin{aligned} *_{p} & =.03 \\
\eta_{\mathrm{p}}{ }^{2} & =.04\end{aligned}$ \\
\hline & \multirow{2}{*}{ RT } & SUD x Congruence & $\begin{array}{l}p=03, \eta_{\mathrm{p}}^{2} \\
=.03\end{array}$ & $\begin{array}{l}p=02 \\
\eta_{p}^{2}=.04\end{array}$ & $\begin{array}{l}p=02 \\
\eta_{p}^{2}=.04\end{array}$ & $\begin{array}{l}p=04 \\
\eta_{p}^{2}=.03\end{array}$ & $\begin{array}{l}p=.09 \\
\eta_{p}^{2}=.02\end{array}$ & $\begin{array}{l}p=.08 \\
\eta_{p}^{2}=.02\end{array}$ \\
\hline & & $\begin{array}{l}\text { SUD x (un)Predictability } \\
\mathrm{x} \text { Congruence }\end{array}$ & $\begin{array}{l}p=.03 \\
\eta_{\mathrm{p}}^{2}=.03\end{array}$ & $\begin{array}{l}p=02 \\
\eta_{\mathrm{p}}^{2}=.04\end{array}$ & $\begin{array}{l}p=.02 \\
\eta_{\mathrm{p}}^{2}=.04\end{array}$ & $\begin{array}{l}p=.02 \\
\eta_{\mathrm{p}}^{2}=.04\end{array}$ & $\begin{array}{l}p=.07 \\
\eta_{p}^{2 .}=.03\end{array}$ & $\begin{array}{l}p=.03 \\
\eta_{\mathrm{p}}^{2}=.04\end{array}$ \\
\hline & \multirow{2}{*}{ Accuracy } & SUD x Overall Threat & $\begin{array}{l}p=.02 \\
\eta_{\mathrm{p}}^{2}=.04\end{array}$ & $\begin{array}{l}p=.05 \\
\eta_{p}^{2}=.03\end{array}$ & $\begin{array}{r}* p=.08 \\
\eta_{p}^{2}=.02\end{array}$ & $\begin{array}{l}p=.03 \\
\eta_{\mathrm{p}}{ }^{2}=.03\end{array}$ & $\begin{array}{l}p=.06 \\
\eta_{p}^{2}=.03\end{array}$ & $\begin{array}{r}{ }^{*} p=.09 \\
\eta_{p}{ }^{2}=.02\end{array}$ \\
\hline & & $\begin{array}{l}\text { SUD x Overall Threat } \mathrm{x} \\
\text { Congruence }\end{array}$ & $\begin{array}{l}p=.05 \\
\eta_{\mathrm{p}}^{2}=.03\end{array}$ & $\begin{array}{l}p=.02 \\
\eta_{\mathrm{p}}^{2}=.04\end{array}$ & $\begin{array}{l}p=.02 \\
\eta_{\mathrm{p}}^{2}=.04\end{array}$ & $\begin{array}{l}\wedge p=.01 \\
\eta_{\mathrm{p}}^{2}=.05\end{array}$ & $\begin{aligned} *_{p} & =.18 \\
\eta_{\mathrm{p}} & =.01\end{aligned}$ & $\begin{array}{l}p=.02 \\
\eta_{\mathrm{p}}^{2}=.04\end{array}$ \\
\hline
\end{tabular}

Note $-*=<.01$ reduction in $\eta_{\mathrm{p}}{ }^{2}, \wedge=>.01$ increase in $\eta_{\mathrm{p}}{ }^{2}$, bolded font $=$ reduced to non-significance. 


\section{CHAPTER FOUR: \\ DISCUSSION}

Despite decades of research suggesting that threat, especially unpredictable threat, induces psychological states associated with breakdown in cognitive control processes, our study was the first to directly examine how predictability of threat impacts neurocognitive and behavioral indicators of cognitive control processes. Further, although emotion-related alterations in cognitive control have long been implicated in disinhibited and impulsive behaviors, threat-related processing has not been measured in relation to these behaviors. Thus, our study was also the first to investigate how reports of engagement in real-life disinhibited and impulsive behaviors (emotion-based rash action, symptoms of alcohol and substance use disorder, self-harm, and aggressive behavior) were associated with threat-induced alterations in cognitive control. We found that while contexts of threat did impact cognitive control, this relationship was dependent upon the relative predictability of the threat as well as individual differences in disinhibition. Interestingly, contrary to our predictions, disinhibition was not necessarily related to threat-induced disruption of cognitive control; rather, in some circumstances, exposure to threat may have actually facilitated cognitive processing of flankers and performance during the task among persons scoring higher on disinhibited behaviors. This study has important implications for understanding the nuanced relationship between threat- 
induced alterations in cognitive processing and the relationship with disinhibited traits and behaviors.

\subsection{Aim 1: Impact of Threat on Cognitive Control}

The first aim of the present study was to examine the impact of threat on neurocognitive (e.g., N2 and P3) and behavioral (e.g., RT, accuracy) indicators of cognitive control using a novel task: a modified threat of shock paradigm (Schmitz \& Grillon, 2012) combined with a cued cognitive control task (Posner et al., 1980). We found expected flanker task effects of slower reaction times, decreased accuracy, and enhanced processing (e.g., more negative frontocentral N2) during incongruent relative to congruent flanker trials. These findings support previous interpretations that the incongruent flankers were more difficult, and thus required more effortful control, compared to congruent flankers and that the frontocentral N2 is particularly sensitive to evaluation of stimulus conflict (Kopp et al., 1996; Nieuwenhuis et al., 2003). However, threat did not seem to relate to neurocognitive indicators of conflict processing (i.e., differential N2/ $\mathrm{P} 3$ processing of flanker congruence trial types), suggesting that threat exposure did not alter the degree of conflict processing, per se. In general, effects of threat on cognitive processing were specific to its relative predictability. That is, predictable relative to unpredictable threat was associated with more early engagement (more negative N2) with flanker task stimuli but this was followed by blunted later-stage cognitive processing (less positive P3) during predictable threat. Meanwhile, behavioral analyses revealed that exposure to threat (vs. no threat) somewhat improved overall accuracy on the task, and this was primarily due to reduced accuracy decrements for incongruent trials in the relatively predictable vs unpredictable blocks.

Taken together, these results provide several novel insights about how exposure to stressor predictability alters cognition, and ultimately, behavior. Our findings suggest that stress 
exposure can influence behavior by altering the overall allocation of top-down cognitive processing resources. By allocating greater processing resources towards early stages of cognitive control in the predictable threat condition, perhaps at the expense of later, more elaborate stages of processing, individuals were able to effectively perform our task under stress. These findings are consistent with previous interpretations that exposure to threat (especially predictable threats) induces heightened vigilance and quick decision making (Starcke \& Brand, 2012, Yu, 2016). Because our task was relatively easy (average accuracy of 97\%), this strategy was "good enough" to result in adequate performance (Oh-Descher, Tanaka, LaBar, Ferrari, Sommer, \& Egner, 2019). However, decision making in real life is complex; thus, it is unclear the extent to which this strategy would be as effective in real-world scenarios. Indeed, Dennis and Chen (2009), using a cued flanker task with threatening and nonthreatening distractor images, found that enhanced N2 amplitudes may actually represent an ineffective compensatory strategy and relate to resource depletion and poorly regulated cognitive control. Others have similarly argued that reduced (less negative) N2 may characterize greater "neural efficiency" (Gray, 2004; Lamm Pine, \& Fox, 2013). Thus, in our study, it is also possible that the heightened $\mathrm{N} 2$ during predictable threat represents neural inefficiency. In other words, caution is warranted when interpreting the meaningfulness of these patterns of neural activation.

Also, of note, we found that adding age into our Aim 1 analyses reduced both the size and significance of most of our effects, with the exception of the effect of overall threat on parietal P3, which was actually larger after accounting for age. We also found a negative association between age and parietal $\mathrm{P}$, which is in line with previous research that the $\mathrm{N} 2$ and P3 amplitudes are decreased with age due to increasing cortical efficiency (Lewis, Lamm, 
Segalowitz, Stieben, \& Zelazo, 2006). As such, future research should consider individual differences in neural efficiency when examining threat-induced alterations in cognition.

\subsection{Aim 2: Associations with Disinhibited Behavior}

The second aim of this project was to examine the associations between cognitive control under threat conditions in the lab and real-world manifestations of disinhibited traits and behaviors. In order to examine whether associations involving disinhibited behaviors were characteristic of specific manifestations, or disinhibited proneness more generally, we used an EFA to extract a general disinhibition factor in order to represent shared liability across the various types of disinhibited behaviors included in our study (i.e., negative urgency, aggressive behavior, self-harm, alcohol and substance use disorder symptoms). Our findings revealed a nuanced relationship between disinhibition and threat-related alterations of cognition under different types of threat.

We found that higher levels of the disinhibition factor were characterized by enhanced P3 amplitudes during more difficult (i.e., incongruent) trials under contexts of threat relative to no threat, and this was accompanied by less incongruence-induced decrements in accuracy during threat vs no threat. Thus, contrary to our predictions, higher levels of disinhibition relate to facilitated processing under conditions of stress, although, as noted, it is unclear whether this strategy of cognitive resource reallocation is indeed "effective" (Gray, 2004). For instance, is possible that because individuals high in trait disinhibition exert similar levels of processing resources regardless of threat (or no threat) context, this may represent a failure to appropriately respond to environmental threat cues. More research is needed in order to determine the meaningfulness of these effects and the implications for real-world ability to regulate behavior under threat among persons with higher disinhibition. 
In addition to our findings related to shared disinhibition, we also found that specific types of disinhibited traits and behaviors were characterized by unique patterns of associations between threat exposure (and the predictability of threat) and neurocognitive and behavioral indicators of cognitive control. For instance, negative urgency, aggressive behavior, and SUD symptoms all showed threat-related enhancements in early engagement with flanker stimuli, suggesting more early vigilance to threat among those high on these behaviors. Specifically, higher levels of both negative urgency and SUD symptoms were associated with more negative $\mathrm{N} 2$ during situations of threat (vs. no threat), and higher levels of aggressive behavior were related to more negative $\mathrm{N} 2$ during predictable (vs. unpredictable) threats. It seems that the general pattern observed in Aim 1, of increased early engagement with the task during threat, was enhanced for persons high on these disinhibited behaviors. Interestingly, for both aggressive behavior and negative urgency, this strategy of threat-induced cognitive reprioritization did not translate into better performance during the task for those scoring higher on these behaviors. Thus, while stress exposure may facilitate engagement with the task (i.e., enhanced N2), perhaps these effects are either too small to meaningfully impact behavior or this reallocation strategy is simply ineffective. Indeed, Rawls and colleagues (2018) argue less neural activation in the face of a negatively charged event (e.g., threat, violence) is more efficient and is therefore less likely to result in an aggressive or impulsive response. Although in our study we also found that greater aggression was associated with generally more blunted $\mathrm{P} 3$ amplitudes, this blunting was less pronounced in the presence of threat. Together, this pattern of heightened engagement during threat without better control over behavior could help explain why certain individuals are more prone to aggressiveness (Verona \& Bresin, 2015). In other words, if aggressive persons are more reactive to perceived slights or potential signs of danger, but do not possess the ability to 
appropriately inhibit aggressive impulses, they are probably more vulnerable to engaging in violent behavior. Finally, we found that high levels of trait negative urgency were associated with reduced N2 discrimination of congruence, which is consistent with the interpretation that individuals with increased propensity towards emotion-induced impulsive behaviors have deficits in cognitive control (Dalley, Everitt, \& Robbins, 2011; Nigg, 2000). Other individual indicators of disinhibited behavior were examined (i.e., self-harm and AUD symptoms), though these effects were relatively small in size and their meaningfulness is unclear.

Lastly, because anxiety often co-occurs with disinhibited behaviors, we ran models including different types of self-reported anxiety as a covariate. Our findings revealed that while anxiety did not seem to account for our findings, it is likely that some of our findings may be due to what disinhibited behaviors share with anxiety, which are also marked by attentional abnormalities and executive functioning deficits (Dennis \& Chen, 2009; Fowles, 2000).

\subsection{Limitations and Strengths}

There are several limitations in the current study. First, given that we could not analyze our physiological measure of affective reactivity (i.e., fear-potentiated startle), we had to rely on participants' self-reported retrospective recall of their experience during the task. This limited our ability to examine the interplay between participants' experience of the threat and any threatinduced alterations in cognition during the task. Next, despite methodological strengths of our paradigm, our task may have been too easy (average accuracy $=97 \%$ ) to sufficiently challenge the cognitive control system. It is possible that with a more demanding task, we may have seen more threat-induced disruption of cognitive control, due to the increased competition for limited processing resources (Pessoa, 2008). 
Despite these limitations, this study had several notable strengths. Our study was the first to directly assess indicators of cognitive control during different shock threat conditions. Indeed, the task design, which combined a threat of shock paradigm (Schmitz \& Grillon, 2012) with a cued cognitive control task (Posner et al., 1980), was highly novel and allowed us to obtain a temporally precise understanding of how the relative predictability of threat impacts cognitive control capabilities. By continuously monitoring cognition under different conditions of threat, we were able to gain new insights regarding the specific cognitive processes that are altered during stressful contexts. Specifically, we found that compared to unpredictable threats, relatively predictable threats induced a pattern of greater early cognitive processing of flankers and subsequently improved performance on the task, suggesting that the effect of threat on cognition depends on both the specific cognitive process and the type of threat. Another major strength of our study includes our focus on applying our results to real-world traits and behaviors. Using both self-report and interviewer-based ratings of disinhibited behaviors allowed us to link our insights about threat-induced alterations in cognition to real-world disinhibited behaviors with high societal burden. Finally, we were able to recruit a diverse, community sample with varying levels of disinhibition, which increases the generalizability of our findings.

\subsection{Future Directions}

In addition to the need to replicate these findings in another sample, future research and theory should seek to understand the psychological processes by which individual differences in the propensity to engage in disinhibited and impulsive behaviors moderate the effect of cognitive processing under different contexts of threat. In particular, future studies should examine other psychological indicators of affective and cognitive processing during contexts of threat, such as the fear-potentiated startle response and the shock-evoked P3 (in order to measure cognitive and 
affective processing of the threat cue) as well as the N1 and P1 (in order to examine how threat alters earlier components that index non-conscious attentional and perceptual processes). Another avenue for future research could be to examine how different aspects of stress might influence cognitive control. For example, instead of manipulating when the shock occurred (threat predictability), one could manipulate the likelihood that the threat will occur (threat uncertainty; Hefner \& Curtin, 2011) or extent of control over the threat exposure (Wood et al., 2015). Indeed, the uncertainty and the controllability over perceived stressors has long been tied to different manifestations of maladaptive behavior and psychopathology (Grupe \& Niitschke, 2013). Finally, future research should investigate cognitive control under stress using a paradigm which is more cognitively taxing (e.g., induces more errors, requires more vigilance; Pessoa, 2008) and potentially more ecologically valid (e.g., gambling task) in order to induce more competition among cognitive resources.

\subsection{Conclusions}

Together, the results of this study demonstrate that threat exposure can alter cognitive control processes, depending on the relative predictability of the threat as well as individual differences in disinhibition. In particular, our findings demonstrate that stress exposure, especially relatively predictable stress, triggers increased processing at early stages of cognitive control, perhaps at the expense of later more elaborative processing. This cognitive reprioritization strategy was related to somewhat better performance during difficult trials in our simple computer task; however, future research is needed to see if this strategy would be as effective in real-world scenarios. Also, though previous literature suggests that threat disrupts cognitive control, especially for individuals prone to engaging in disinhibited behaviors, we did not necessarily find this. Instead, threat increases engagement with task for persons high on 
disinhibited behaviors, although it does not necessarily make their performance better. This research thus expands on what is known about threat processing and linked it to high risk behaviors with high societal burden. Perhaps a greater understanding of the nuanced relationship between threat-induced alterations in cognitive processing and disinhibited traits and behaviors could lead to more effective utilization of therapeutic techniques. 


\section{REFERENCES}

Adhikari, A. (2014). Distributed circuits underlying anxiety. Frontiers in behavioral neuroscience, $8,112$.

Adolphs, R. (2013). The biology of fear. Current Biology, 23(2), R79-R93.

Aiken, L. S., \& West, S. G. (1991). Multiple regression: Testing and interpreting interactions. Newbury Park, London, Sage.

American Psychiatric Association. (2013). Diagnostic and statistical manual of mental disorders (DSM-5®). Arlington, VA: American Psychiatric Pub.

Anestis, M. D., \& Joiner, T. E. (2011). Examining the role of emotion in suicidality: Negative urgency as an amplifier of the relationship between components of the interpersonalpsychological theory of suicidal behavior and lifetime number of suicide attempts. Journal of affective disorders, 129(1-3), 261-269.

Anestis, M. D., Smith, A. R., Fink, E. L., \& Joiner, T. E. (2009). Dysregulated eating and distress: Examining the specific role of negative urgency in a clinical sample. Cognitive Therapy and Research, 33(4), 390-397.

Arnsten, A. F. (1998). The biology of being frazzled. Science, 280(5370), 1711-1712.

Baas, J. M. P., Kenemans, J. L., Böcker, K. B. E., \& Verbaten, M. N. (2002). Threat-induced cortical processing and startle potentiation. Neuroreport, 13(1), 133-137. 
Baldwin, S. A., Larson, M. J., \& Clayson, P. E. (2015). The dependability of electrophysiological measurements of performance monitoring in a clinical sample: A generalizability and decision analysis of the ERN and P e. Psychophysiology, 52(6), 790800.

Baler, R. D., \& Volkow, N. D. (2006). Drug addiction: the neurobiology of disrupted selfcontrol. Trends in molecular medicine, 12(12), 559-566.

Bari, A., \& Robbins, T. W. (2013). Inhibition and impulsivity: behavioral and neural basis of response control. Progress in neurobiology, 108, 44-79.

Baumeister, S., Hohmann, S., Wolf, I., Plichta, M. M., Rechtsteiner, S., Zangl, M., ... \& Holtmann, M. (2014). Sequential inhibitory control processes assessed through simultaneous EEG-fMRI. Neuroimage, 94, 349-359.

Beck, D. M., \& Kastner, S. (2009). Top-down and bottom-up mechanisms in biasing competition in the human brain. Vision research, 49(10), 1154-1165.

Bennett, K. P., Dickmann, J. S., \& Larson, C. L. (2018). If or when? Uncertainty's role in anxious anticipation. Psychophysiology, e13066.

Blumenthal, T. D., Cuthbert, B. N., Filion, D. L., Hackley, S., Lipp, O. V., \& Van Boxtel, A. (2005). Committee report: Guidelines for human startle eyeblink electromyographic studies. Psychophysiology, 42(1), 1-15

Botvinick, M. M., Braver, T. S., Barch, D. M., Carter, C. S., \& Cohen, J. D. (2001). Conflict monitoring and cognitive control. Psychological review, 108(3), 624.

Boyce, W. T., \& Ellis, B. J. (2005). Biological sensitivity to context: I. An evolutionarydevelopmental theory of the origins and functions of stress reactivity. Development and psychopathology, 17(2), 271-301. 
Bredemeier, K., \& Berenbaum, H. (2008). Intolerance of uncertainty and perceived threat. Behaviour Research and Therapy, 46(1), 28-38.

Bradford, D. E., Kaye, J. T., \& Curtin, J. J. (2014). Not just noise: Individual differences in general startle reactivity predict startle response to uncertain and certain threat. Psychophysiology, 51(5), 407-411.

Bradford, D. E., Magruder, K. P., Korhumel, R. A., \& Curtin, J. J. (2014). Using the threat probability task to assess anxiety and fear during uncertain and certain threat. Journal of visualized experiments: JoVE, (91).

Brady, K. T., \& Sinha, R. (2005). Co-occurring mental and substance use disorders: the neurobiological effects of chronic stress. American Journal of Psychiatry, 162(8), 14831493.

Brown, G. L., Ebert, M. H., Goyer, P. F., Jimerson, D. C., Klein, W. J., Bunney, W. E., \& Goodwin, F. K. (1982). Aggression, suicide, and serotonin: relationships of CSF amine metabolites. The American Journal of Psychiatry.

Brown, M. R., Lebel, R. M., Dolcos, F., Wilman, A. H., Silverstone, P. H., Pazderka, H., ... \& Zedkova, L. (2012). Effects of emotional context on impulse control. Neuroimage, 63(1), 434-446.

Brydges, C. R., Clunies-Ross, K., Clohessy, M., Lo, Z. L., Nguyen, A., Rousset, C., ... \& Fox, A. M. (2012). Dissociable components of cognitive control: an event-related potential (ERP) study of response inhibition and interference suppression. PloS one, 7(3), e34482.

Carretié, L., Martín-Loeches, M., Hinojosa, J. A., \& Mercado, F. (2001). Emotion and attention interaction studied through event-related potentials. Journal of cognitive neuroscience, 13(8), 1109-1128. 
Clayson, P. E., \& Larson, M. J. (2013). Psychometric properties of conflict monitoring and conflict adaptation indices: Response time and conflict N 2 event- related potentials. Psychophysiology, 50(12), 1209-1219.

Clayson, P. E., \& Miller, G. A. (2017). ERP Reliability Analysis (ERA) Toolbox: An opensource toolbox for analyzing the reliability of event-related brain potentials. International Journal of Psychophysiology, 111, 68-79.

Coccaro, E. F., Berman, M. E., \& Kavoussi, R. J. (1997). Assessment of life history of aggression: development and psychometric characteristics. Psychiatry research, 73(3), $147-157$

Cohen, N., \& Henik, A. (2012). Do irrelevant emotional stimuli impair or improve executive control?. Frontiers in Integrative Neuroscience, 6, 33.

Cuthbert, B. N., \& Insel, T. R. (2013). Toward the future of psychiatric diagnosis: the seven pillars of RDoC. BMC medicine, 11(1), 126.

Cyders, M. A., Littlefield, A. K., Coffey, S., \& Karyadi, K. A. (2014). Examination of a short English version of the UPPS-P Impulsive Behavior Scale. Addictive behaviors, 39(9), $1372-1376$.

Dalley, J. W., Everitt, B. J., \& Robbins, T. W. (2011). Impulsivity, compulsivity, and top-down cognitive control. Neuron, 69(4), 680-694.

Davis, M. (2006). Neural systems involved in fear and anxiety measured with fear-potentiated startle. American Psychologist, 61(8), 741.

Davis, M., Walker, D. L., Miles, L., \& Grillon, C. (2010). Phasic vs sustained fear in rats and humans: role of the extended amygdala in fear vs anxiety. Neuropsychopharmacology, $35(1), 105$ 
Davidson, R. J., Putnam, K. M., \& Larson, C. L. (2000). Dysfunction in the neural circuitry of emotion regulation--a possible prelude to violence. science, 289(5479), 591-594.

Delorme, A., \& Makeig, S. (2004). EEGLAB: an open source toolbox for analysis of single-trial EEG dynamics including independent component analysis. Journal of neuroscience methods, 134(1), 9-21.

Dennis, T. A., \& Chen, C. C. (2009). Trait anxiety and conflict monitoring following threat: an ERP study. Psychophysiology, 46(1), 122-131.

Diamond, A. (2013). Executive functions. Annual review of psychology, 64, 135-168.

Donchin, E. Dierolf, A. M., Fechtner, J., Böhnke, R., Wolf, O. T., \& Naumann, E. (2017). Influence of acute stress on response inhibition in healthy men: An ERP study. Psychophysiology, 54(5), 684-695.

Engelmann, J. B., \& Hare, T. A (2018). Emotions can bias decision-making processes by promoting specific behavioral tendencies or motivational drives. In A. S. Fox, R.C. Lapate, A. J. Shackman, \& R. J. Davidson (Eds). The Nature of Emotion. Fundamental questions. (2nd edition). New York: Oxford University Press.

Eriksen, B. A., \& Eriksen, C. W. (1974). Effects of noise letters upon the identification of a target letter in a nonsearch task. Perception \& psychophysics, 16(1), 143-149.

Etkin, A., Büchel, C., \& Gross, J. J. (2015). The neural bases of emotion regulation. Nature reviews neuroscience, 16(11), 693.

Fan, J., McCandliss, B. D., Sommer, T., Raz, A., \& Posner, M. I. (2002). Testing the efficiency and independence of attentional networks. Journal of cognitive neuroscience, 14(3), 340347. 
Faul, F., Erdfelder, E., Lang, A. G., \& Buchner, A. (2007). G* Power 3: A flexible statistical power analysis program for the social, behavioral, and biomedical sciences. Behavior research methods, 39(2), 175-191.

Ferree, T. C., Luu, P., Russell, G. S., \& Tucker, D. M. (2001). Scalp electrode impedance, infection risk, and EEG data quality. Clinical Neurophysiology, 112(3), 536-544.

Finy, M. S., Bresin, K., Korol, D. L., \& Verona, E. (2014). Impulsivity, risk taking, and cortisol reactivity as a function of psychosocial stress and personality in adolescents. Development and psychopathology, 26(4pt1), 1093-1111.

Folstein, J. R., \& Van Petten, C. (2008). Influence of cognitive control and mismatch on the N2 component of the ERP: a review. Psychophysiology, 45(1), 152-170.

Fowles, D. C. (2000). Electrodermal hyporeactivity and antisocial behavior: does anxiety mediate the relationship?. Journal of affective disorders, 61(3), 177-189.

Gamboz, N., Zamarian, S., \& Cavallero, C. (2010). Age-related differences in the attention network test (ANT). Experimental aging research, 36(3), 287-305.

Garcia-Garcia, M., Domínguez-Borràs, J., SanMiguel, I., \& Escera, C. (2008).

Electrophysiological and behavioral evidence of gender differences in the modulation of distraction by the emotional context. Biological Psychology, 79(3), 307-316.

Gillis, M. M., Haaga, D. A., \& Ford, G. T. (1995). Normative values for the Beck Anxiety Inventory, Fear Questionnaire, Penn State Worry Questionnaire, and Social Phobia and Anxiety Inventory. Psychological Assessment, 7(4), 450.

Goldberg, L. S., \& Grandey, A. A. (2007). Display rules versus display autonomy: emotion regulation, emotional exhaustion, and task performance in a call center simulation. Journal of occupational health psychology, 12(3), 301. 
Gorka, S. M., Lieberman, L., Phan, K. L., \& Shankman, S. A. (2016). Association between problematic alcohol use and reactivity to uncertain threat in two independent samples. Drug and alcohol dependence, 164, 89-96.

Gorka, S. M., Nelson, B. D., \& Shankman, S. A. (2013). Startle response to unpredictable threat in comorbid panic disorder and alcohol dependence. Drug and alcohol dependence, 132(1-2), 216-222.

Grant, B. F., Stinson, F. S., Dawson, D. A., Chou, S. P., Dufour, M. C., Compton, W., ... \& Kaplan, K. (2004). Prevalence and co-occurrence of substance use disorders and independentmood and anxiety disorders: Results from the national epidemiologic survey on alcohol and relatedconditions. Archives of general psychiatry, 61(8), 807-816.

Gray, J. R. (2004). Integration of emotion and cognitive control. Current directions in psychological science, 13(2), 46-48.

Grillon, C. (2008). Models and mechanisms of anxiety: evidence from startle studies. Psychopharmacology, 199(3), 421-437.

Grillon, C., Baas, J. P., Lissek, S., Smith, K., \& Milstein, J. (2004). Anxious responses to predictable and unpredictable aversive events. Behavioral neuroscience, 118(5), 916.

Grillon, C., Pine, D. S., Lissek, S., Rabin, S., Bonne, O., \& Vythilingam, M. (2009). Increased anxiety during anticipation of unpredictable aversive stimuli in posttraumatic stress disorder but not in generalized anxiety disorder. Biological psychiatry, 66(1), 47-53.

Groom, M. J., \& Cragg, L. (2015). Differential modulation of the N2 and P3 event-related potentials by response conflict and inhibition. Brain and cognition, 97, 1-9.

Grupe, D. W., \& Nitschke, J. B. (2013). Uncertainty and anticipation in anxiety: an integrated neurobiological and psychological perspective. Nature Reviews Neuroscience, 14(7), 488. 
Hefner, K. R., \& Curtin, J. J. (2012). Alcohol stress response dampening: selective reduction of anxiety in the face of uncertain threat. Journal of psychopharmacology, 26(2), 232-244.

Heil, M., Osman, A., Wiegelmann, J., Rolke, B., \& Hennighausen, E. (2000). N200 in the Eriksen-task: Inhibitory executive process?. Journal of Psychophysiology, 14(4), 218.

Hermans, E. J., Henckens, M. J., Joëls, M., \& Fernández, G. (2014). Dynamic adaptation of large-scale brain networks in response to acute stressors. Trends in neurosciences, 37(6), 304-314.

Herry, C., Bach, D. R., Esposito, F., Di Salle, F., Perrig, W. J., Scheffler, K., ... \& Seifritz, E. (2007). Processing of temporal unpredictability in human and animal amygdala. Journal of Neuroscience, 27(22), 5958-5966.

Hsu, M., Bhatt, M., Adolphs, R., Tranel, D., \& Camerer, C. F. (2005). Neural systems responding to degrees of uncertainty in human decision-making. Science, 310(5754), $1680-1683$.

Iacono, W. G., Carlson, S. R., Taylor, J., Elkins, I. J., \& McGue, M. (1999). Behavioral disinhibition and the development of substance-use disorders: findings from the Minnesota Twin Family Study. Development and psychopathology, 11(4), 869-900.

Jiang, C., \& Rau, P. L. P. (2017). The detrimental effect of acute stress on response inhibition when exposed to acute stress: an event-related potential analysis. NeuroReport, 28(14), 922-928.

Joëls, M., \& Baram, T. Z. (2009). The neuro-symphony of stress. Nature reviews neuroscience, 10(6), 459 . 
Kaiser, A. J., Milich, R., Lynam, D. R., \& Charnigo, R. J. (2012). Negative urgency, distress tolerance, and substance abuse among college students. Addictive behaviors, 37(10), 1075-1083.

Kałamała, P., Szewczyk, J., Senderecka, M., \& Wodniecka, Z. (2018). Flanker task with equiprobable congruent and incongruent conditions does not elicit the conflict N2. Psychophysiology, 55(2), e12980.

Kanske, P. (2012). On the influence of emotion on conflict processing. Frontiers in Integrative Neuroscience, 6, 42.

Kaye, J. T., Bradford, D. E., \& Curtin, J. J. (2016). Psychometric properties of startle and corrugator response in NPU, affective picture viewing, and resting state tasks. Psychophysiology, 53(8), 1241-1255.

Keene, O. N. (1995). The log transformation is special. Statistics in medicine, 14(8), 811-819.

Koechlin, E., \& Summerfield, C. (2007). An information theoretical approach to prefrontal executive function. Trends in cognitive sciences, 11(6), 229-235.

Koo, T. K., \& Li, M. Y. (2016). A guideline of selecting and reporting intraclass correlation coefficients for reliability research. Journal of chiropractic medicine, 15(2), 155-163.

Koolhaas, J. M., Bartolomucci, A., Buwalda, B., de Boer, S. F., Flügge, G., Korte, S. M., ... Fuchs, E. (2011). Stress revisited: A critical evaluation of the stress concept. Neuroscience \& Biobehavioral Reviews, 35(5), 1291-1301.

Kopp, B., Rist, F., \& Mattler, U. W. E. (1996). N200 in the flanker task as a neurobehavioral tool for investigating executive control. Psychophysiology, 33(3), 282-294.

Kring, A. M., \& Bachorowski, J. A. (1999). Emotions and psychopathology. Cognition \& Emotion, 13(5), 575-599. 
Lake, J. I., \& LaBar, K. S. (2011). Unpredictability and uncertainty in anxiety: a new direction for emotional timing research. Frontiers in integrative neuroscience, 5, 55.

Lamm, C., Pine, D. S., \& Fox, N. A. (2013). Impact of negative affectively charged stimuli and response style on cognitive-control-related neural activation: an ERP study. Brain and cognition, 83(2), 234-243.

Larsen, R. J., Diener, E., \& Emmons, R. A. (1986). Affect intensity and reactions to daily life events. Journal of personality and social psychology, 51(4), 803.

LeDoux, J. E. (2012). Evolution of human emotion: a view through fear. In Progress in brain research, 195, 431-442.

LeDoux, J. E., \& Pine, D. S. (2016). Using neuroscience to help understand fear and anxiety: a two-system framework. American Journal of Psychiatry, 173(11), 1083-1093.

Lewis, M. D., Lamm, C., Segalowitz, S. J., Stieben, J., \& Zelazo, P. D. (2006).

Neurophysiological correlates of emotion regulation in children and adolescents. Journal of cognitive neuroscience, 18(3), 430-443.

Liston, C., McEwen, B. S., \& Casey, B. J. (2009). Psychosocial stress reversibly disrupts prefrontal processing and attentional control. Proceedings of the National Academy of Sciences, 106(3), 912-917.

Littlefield, A. K., \& Sher, K. J. (2010). The multiple, distinct ways that personality contributes to alcohol use disorders. Social and personality psychology compass, 4(9), 767-782.

Lynam, D. R., Smith, G. T., Whiteside, S. P., \& Cyders, M. A. (2006). The UPPS-P: Assessing five personality pathways to impulsive behavior. West Lafayette, IN: Purdue University. 
McKenna, F. P., \& Sharma, D. (1995). Intrusive cognitions: An investigation of the emotional Stroop task. Journal of Experimental Psychology: Learning, Memory, and Cognition, 21(6), 1595.

MacNamara, A., \& Barley, B. (2018). Event- related potentials to threat of predictable and unpredictable shock. Psychophysiology, 55(10), e13206.

McEwen, B. S., \& Stellar, E. (1993). Stress and the individual: mechanisms leading to disease. Archives of internal medicine, 153(18), 2093-2101.

Menon, V. (2011). Large-scale brain networks and psychopathology: a unifying triple network model. Trends in cognitive sciences, 15(10), 483-506.

Menon, V. (2015). Salience Network. In Brain Mapping: An Encyclopedic Reference (Arthur W. Toga, editor). Academic Press: Elsevier, 2, 597-611.

Meyer, T. J., Miller, M. L., Metzger, R. L., \& Borkovec, T. D. (1990). Development and validation of the penn state worry questionnaire. Behavior Research and Therapy, 28, 487-495.

Miller, E. K., \& Cohen, J. D. (2001). An integrative theory of prefrontal cortex function. Annual review of neuroscience, 24(1), 167-202.

Miyake, A., \& Friedman, N. P. (2012). The nature and organization of individual differences in executive functions: Four general conclusions. Current directions in psychological science, 21(1), 8-14.

Mullins- Sweatt, S. N., Lengel, G. J., \& Grant, D. M. (2013). Non- suicidal self- injury: The contribution of general personality functioning. Personality and mental health, 7(1), 5668. 
Muraven, M., Tice, D. M., \& Baumeister, R. F. (1998). Self-control as a limited resource: Regulatory depletion patterns. Journal of personality and social psychology, 74(3), 774.

Nee, D. E., Wager, T. D., \& Jonides, J. (2007). Interference resolution: insights from a metaanalysis of neuroimaging tasks. Cognitive, Affective, \& Behavioral Neuroscience, 7(1), 117.

Nelson, B. D., \& Hajcak, G. (2017). Defensive motivation and attention in anticipation of different types of predictable and unpredictable threat: A startle and event- related potential investigation. Psychophysiology, 54(8), 1180-1194.

Nelson, B. D., Hajcak, G., \& Shankman, S. A. (2015). Event- related potentials to acoustic startle probes during the anticipation of predictable and unpredictable threat. Psychophysiology, 52(7), 887-894.

Nelson, L. D., Patrick, C. J., \& Bernat, E. M. (2011). Operationalizing proneness to externalizing psychopathology as a multivariate psychophysiological phenotype. Psychophysiology, 48(1), 64-72.

Nelson, L. D., Strickland, C., Krueger, R. F., Arbisi, P. A., \& Patrick, C. J. (2016). Neurobehavioral traits as transdiagnostic predictors of clinical problems. Assessment, 23(1), 75-85.

Neuhaus, A. H., Urbanek, C., Opgen-Rhein, C., Hahn, E., Ta, T. M. T., Koehler, S., Gross, M., \& Dettling, M. (2010). Event-related potentials associated with Attention Network Test. International Journal of Psychophysiology, 76(2), 72-79. 
Nieuwenhuis, S., Yeung, N., Van Den Wildenberg, W., \& Ridderinkhof, K. R. (2003).

Electrophysiological correlates of anterior cingulate function in a go/no-go task: effects of response conflict and trial type frequency. Cognitive, affective, \& behavioral neuroscience, 3(1), 17-26.

Nigg, J. T. (2000). On inhibition/disinhibition in developmental psychopathology: views from cognitive and personality psychology and a working inhibition taxonomy. Psychological bulletin, 126(2), 220.

Nock, M. K., Holmberg, E. B., Photos, V. I., \& Michel, B. D. (2007). Self-injurious thoughts and behaviors interview: Development, reliability, and validity in an adolescent sample. Psychological Assessment, 19(3), 309-317.

Ochsner, K. N., \& Gross, J. J. (2005). The cognitive control of emotion. Trends in cognitive sciences, 9(5), 242-249.

Oh-Descher, H., Tanaka, H., LaBar, K. S., Ferrari, S., Sommer, M. A., \& Egner, T. (2019). Anticipatory anxiety promotes satisficing during multi-cue probabilistic decision making.

Öhman, A. (2008). Fear and anxiety. Emotions, 709.

Patel, S. H., \& Azzam, P. N. (2005). Characterization of N200 and P300: selected studies of the event-related potential. International journal of medical sciences, 2(4), 147.

Pessoa, L. (2008). On the relationship between emotion and cognition. Nature reviews neuroscience, $9(2), 148$.

Pessoa, L. (2009). How do emotion and motivation direct executive control?. Trends in cognitive sciences, 13(4), 160-166.

Petersen, I. T. (2016). Neurophysiological Mechanisms in the Development of Externalizing Behavior Problems in Young Children. 
Polich, J. (2007). Updating P300: an integrative theory of P3a and P3b. Clinical neurophysiology, 118(10), 2128-2148.

Posner, K., Brent, D., Lucas, C., Gould, M., Stanley, B., Brown, G., Fisher, P.; Zelazny, J. Burke, A.; Oquendo, M.; \& Mann, J. (2008). Columbia-suicide severity rating scale (CSSRS). New York, NY: Columbia University Medical Center.

Posner, M. I., Snyder, C. R., \& Davidson, B. J. (1980). Attention and the detection of signals. Journal of experimental psychology: General, 109(2), 160.

Potts, G. F., Martin, L. E., Burton, P., \& Montague, P. R. (2006). When things are better or worse than expected: the medial frontal cortex and the allocation of processing resources. Journal of cognitive neuroscience, 18(7), 1112-1119.

Quarantelli, E. L. (1954). The nature and conditions of panic. American Journal of Sociology, 60(3), 267-275.

Rawls, E., Jabr, M. M., Moody, S. N., \& Lamm, C. (2018). Neural mechanisms underlying the link between effortful control and aggression: An ERP study. Neuropsychologia, 117, 302-310.

Rietdijk, W. J., Franken, I. H., \& Thurik, A. R. (2014). Internal consistency of event-related potentials associated with cognitive control: N2/P3 and ERN/Pe PloS one, 9(7), e102672.

Robinson, M. D., Watkins, E. R., \& Harmon-Jones, E. (Eds.). (2013). Handbook of cognition and emotion. Guilford Press.

Robinson, O. J., Vytal, K., Cornwell, B. R., \& Grillon, C. (2013). The impact of anxiety upon cognition: perspectives from human threat of shock studies. Frontiers in Human Neuroscience, 7, 203. 
Sandi, C. (2013). Stress and cognition. Wiley Interdisciplinary Reviews: Cognitive Science, 4(3), 245-261.

Sapolsky, R. M. (1994). Individual differences and the stress response.

Sapolsky, R. M. (2004). Why zebras don't get ulcers: The acclaimed guide to stress, stressrelated diseases, and coping-now revised and updated. Holt paperbacks.

Sarinopoulos, I., Grupe, D. W., Mackiewicz, K. L., Herrington, J. D., Lor, M., Steege, E. E., \& Nitschke, J. B. (2009). Uncertainty during anticipation modulates neural responses to aversion in human insula and amygdala. Cerebral Cortex, 20(4), 929-940.

Schiller, D., \& Delgado, M. R. (2010). Overlapping neural systems mediating extinction, reversal and regulation of fear. Trends in cognitive sciences, 14(6), 268-276.

Schmitz, A., \& Grillon, C. (2012). Assessing fear and anxiety in humans using the threat of predictable and unpredictable aversive events (the NPU-threat test). Nature Protocols, 7(3), 527.

Settles, R. E., Fischer, S., Cyders, M. A., Combs, J. L., Gunn, R. L., \& Smith, G. T. (2012). Negative urgency: A personality predictor of externalizing behavior characterized by neuroticism, low conscientiousness, and disagreeableness. Journal of abnormal psychology, 12l(1), 160.

Shankman, S. A., Nelson, B. D., Sarapas, C., Robison-Andrew, E. J., Campbell, M. L., Altman, S. E., ... \& Gorka, S. M. (2013). A psychophysiological investigation of threat and reward sensitivity in individuals with panic disorder and/or major depressive disorder. Journal of abnormal psychology, 122(2), 322 . 
Sheehan, D. V. (2014). Mini-International Neuropsychiatric Interview (MINI) English Version 7.0.0 for DSM-5. Tampa, FL: University of South Florida, Institute for Research in Psychiatry.

Sprague, J., \& Verona, E. (2010). Emotional conditions disrupt behavioral control among individuals with dysregulated personality traits. Journal of Abnormal Psychology, 119(2), 409.

Starcke, K., \& Brand, M. (2012). Decision making under stress: a selective review. Neuroscience \& Biobehavioral Reviews, 36(4), 1228-1248.

Stevens, A. K., Blanchard, B. E., \& Littlefield, A. K. (2018). Impulsive dispositions and alcohol: what we know, how we know it, and where to go from here. Borderline personality disorder and emotion dysregulation, 5(1), 4.

Stewart, J. L., Silton, R. L., Sass, S. M., Fisher, J. E., Edgar, J. C., Heller, W., \& Miller, G. A. (2010). Attentional bias to negative emotion as a function of approach and withdrawal anger styles: an ERP investigation. International Journal of Psychophysiology, 76(1), 918.

Stoet, G. (2010). Sex differences in the processing of flankers. Quarterly Journal of Experimental Psychology, 63(4), 633-638.

Stormark, K. M., Nordby, H., \& Hugdahl, K. (1995). Attentional shifts to emotionally charged cues: Behavioural and ERP data. Cognition \& Emotion, 9(5), 507-523.

Swick, D., Ashley, V., \& Turken, U. (2011). Are the neural correlates of stopping and not going identical? Quantitative meta-analysis of two response inhibition tasks. Neuroimage, 56(3), 1655-1665. 
Taylor, S. F., \& Liberzon, I. (2007). Neural correlates of emotion regulation in psychopathology. Trends in cognitive sciences, 11(10), 413-418.

Tice, D. M., Bratslavsky, E., \& Baumeister, R. F. (2001). Emotional distress regulation takes precedence over impulse control: If you feel bad, do it!. In Self-Regulation and SelfControl (pp. 275-306). Routledge.

Torrisi, S., Gorka, A. X., Gonzalez-Castillo, J., O’Connell, K., Balderston, N., Grillon, C., \& Ernst, M. (2018). Extended amygdala connectivity changes during sustained shock anticipation. Translational psychiatry, 8(1), 33.

Vaidyanathan, U., Patrick, C. J., \& Cuthbert, B. N. (2009). Linking dimensional models of internalizing psychopathology to neurobiological systems: Affect-modulated startle as an indicator of fear and distress disorders and affiliated traits. Psychological bulletin, 135(6), 909.

Venables, N. C., Sellbom, M., Sourander, A., Kendler, K. S., Joiner, T. E., Drislane, L. E., Sillanmäki, L., Elonheimo, H., Parkkola, K., Multimaki, P., .. Patrick, C. J. (2015). Separate and interactive contributions of weak inhibitory control and threat sensitivity to prediction of suicide risk. Psychiatry research, 226(2-3), 461-6.

Verona, E., \& Bresin, K. (2015). Aggression proneness: Transdiagnostic processes involving negative valence and cognitive systems. International Journal of Psychophysiology, $98(2), 321-329$.

Verona, E., \& Kilmer, A. (2007). Stress exposure and affective modulation of aggressive behavior in men and women. Journal of abnormal psychology, 116(2), 410. 
Verona, E., Sprague, J., \& Sadeh, N. (2012). Inhibitory control and negative emotional processing in psychopathy and antisocial personality disorder. Journal of abnormal psychology, 121(2), 498.

Volpe, U., Mucci, A., Bucci, P., Merlotti, E., Galderisi, S., \& Maj, M. (2007). The cortical generators of P3a and P3b: a LORETA study. Brain research bulletin, 73(4-6), 220-230.

Walker, D. L., \& Davis, M. (2008). Role of the extended amygdala in short-duration versus sustained fear: a tribute to Dr. Lennart Heimer. Brain Structure and Function, 213(1-2), $29-42$.

Watson, D., \& Clark, L. A. (1991). The mood and anxiety symptom questionnaire. Unpublished manuscript, University of Iowa, department of psychology, Iowa City.

Watson, D., Clark, L. A., \& Tellegen, A. (1988). Development and validation of brief measures of positive and negative affect: the PANAS scales. Journal of personality and social psychology, 54(6), 1063.

Watson, D., Clark, L. A., Weber, K., Assenheimer, J. S., Strauss, M. E., \& McCormick, R. A. (1995). Testing a tripartite model: II. Exploring the symptom structure of anxiety and depression in student, adult, and patient samples. Journal of abnormal Psychology, 104(1), 15

Weinberg, A., Meyer, A., Hale- Rude, E., Perlman, G., Kotov, R., Klein, D. N., \& Hajcak, G. (2016). Error- related negativity (ERN) and sustained threat: Conceptual framework and empirical evaluation in an adolescent sample. Psychophysiology, 53(3), 372-385.

Wood, K. H., Wheelock, M. D., Shumen, J. R., Bowen, K. H., Ver Hoef, L. W., \& Knight, D. C. (2015). Controllability modulates the neural response to predictable but not unpredictable threat in humans. NeuroImage, 119, 371-381. 
Xie, L., Ren, M., Cao, B., \& Li, F. (2017). Distinct brain responses to different inhibitions: Evidence from a modified Flanker Task. Scientific reports, 7(1), 6657.

Yancey, J. R., Venables, N. C., \& Patrick, C. J. (2016). Psychoneurometric operationalization of threat sensitivity: Relations with clinical symptom and physiological response criteria. Psychophysiology, 53(3), 393-405.

Yu, R. (2016). Stress potentiates decision biases: A stress induced deliberation-to-intuition (SIDI) model. Neurobiology of stress, 3, 83-95.

Zapolski, T. C., Cyders, M. A., \& Smith, G. T. (2009). Positive urgency predicts illegal drug use and risky sexual behavior. Psychology of Addictive Behaviors, 23(2), 348. 
APPENDIX A.

Recruitment Materials (Part I- Flyer)

\title{
USF

\section{PARTICIPANTS NEEDED FOR PAID RESEARCH STUDY}

\author{
You can earn up to $\$ 110$ for participating in two sessions \\ totaling 5 hours!
}

Researchers at USF are looking for adults to participate in a two-part research study on the effects of emotion and behavior on decision-making. Your participation will help us further knowledge in this important area.

\section{N T E R E S T E D?}

C A L L : ( 813 ) $974-4393$

E M A I L: U S F E B L A B @ G M A I L . C O M SAY THAT YOU ARE CALLING ABOUT THE TACOS STUDY. 


\section{APPENDIX B. \\ Recruitment Materials (Part II - Advertisement) \\ Craigslist/ Facebook/ Newspaper Advertisements:}

(USF IRB \#Pro00027233)

USF Psychology researchers seeking adults (18-30 years old) to participate in a 3-hour study on the effects of emotions on decision-making. The study consists of two sessions that are 2.5 hours each, several days apart. Payment: \$35 (and transportation costs) and opportunity to participate in another paid study. Call (813) 974 - 4393 or email usfeblab@gmail.com 


\section{APPENDIX C. \\ Pre-screening consent for phone screening script}

"Hello, my name is [

7. I am a member of the research team at the University of South Florida. First, thank you for your interest in our Study. The full name of our study is the Transdiagnostic Affective and Cognitive Systems study, but we call it the TACoS study for short. Before I tell you more about the study, I need to ask you a series of screening questions in order to determine whether you are eligible to participate. Do you have 5 minutes available at this time so that we can go through this process?"

If the person says NO: "Okay, that is not a problem. We can reschedule this call for a time that is more convenient for you. When will be the best time for us to call you?"

If the person say YES: "Great! We are going to move on to the screening questions. If you do not understand a question or need me to clarify something about a question, feel free to stop me and ask."

\begin{tabular}{|c|l|}
\hline \multicolumn{1}{|c|}{ Screening Questions } & \multicolumn{1}{|c|}{ Criteria } \\
\hline $\begin{array}{l}\text { Are you a college student? } \\
\text { Do you attend college full time? } \\
\text { Are you a USF employee? }\end{array}$ & $\begin{array}{l}\text { No full-time } \\
\text { college/university } \\
\text { students. } \\
\text { Part-time students in } \\
\text { community colleges, } \\
\text { technical schools etc. } \\
\text { are acceptable. } \\
\text { No USF employees. }\end{array}$ \\
\hline $\begin{array}{l}\text { How old are you? What is your date of birth? } \\
\text { Do you speak and read English well? }\end{array}$ & 18 - 40 \\
\hline $\begin{array}{l}\text { Have you ever heard voices or seen things that other people cannot? } \\
\text { If Yes you have visions or see things that other people } \\
\text { couldn't see? } \\
\text { What about strange sensations on your skin, like } \\
\text { feeling like something is creeping or crawling on or } \\
\text { under your skin? How about the feeling of being } \\
\text { touched or stroked? }\end{array}$ & $\begin{array}{l}\text { No history of } \\
\text { schizophrenia }\end{array}$ \\
\hline
\end{tabular}


What about having unusual sensations inside a part of your body, like a feeling of electricity?

How about eating or drinking something that you thought tasted bad or strange even though everyone else who tasted it thought it was fine?

What about smelling unpleasant things that other people couldn't smell, like decaying food or dead bodies?

Have you ever experienced extreme beliefs that people or organizations are out to get you or that you are a very famous person?

\section{If Yes (For Delusions):}

-Has it ever seemed like people were talking about you or taking special notice of you?

If Yes:

-Were you convinced they were talking about you or did you think it might have been your imagination? -Did you ever have the feeling that something on the radio, $T V$, or in a movie was meant especially for you? -Did you ever have the feeling that the words in a popular song were meant to send you a special message?

-Did you ever have the feeling that what people were wearing was intended to send you a special message? -Did you ever have the feeling that street signs or billboards had a special meaning for you?

-What about anyone going out of their way to give you a hard time, or trying to hurt you?

-Have you ever had the feeling that you were being followed, spied on, manipulated or plotted against? -Did you ever have the feeling that you were being poisoned or that your food had been tampered with? -Have you ever thought that you were especially important in some way, or that you had special powers or knowledge?

-Did you ever believe that you had a special or close relationship with a celebrity or someone else famous? -Have you ever been convinced that something was very wrong with your physical health even though your doctor said nothing was wrong...like you had cancer or some other disease?

-Have you ever felt that something strange was happening to parts of your body? 
-Have you ever felt that you had committed a crime or done something terrible for which you should be punished?

-Have you ever felt that something you did, or should have done but did not do, caused serious harm to your parents, children, other family members, or friends?

-What about feeling responsible for a disaster such as a fire, flood, or earthquake?

-Have you ever been convinced that your spouse or partner was being unfaithful to you?

-If Yes: How did you know?

-Did you ever have a "secret admirer" who, when you tried to contact them, denied that they were in love with you?

-Are you a religious or spiritual person?

-If YES: Have you ever had any religious or spiritual experiences that the other people in your religious or spiritual community have not experienced?

-If YES: Tell me about your experiences?

-If NO: Have you ever felt that God, the devil, or some other spiritual being or higher power has communicated directly with you? -Did you ever feel that someone or something outside yourself was controlling your thoughts or actions against your will?

-Did you ever feel that certain thoughts that were not your own were put into your head?

-What about thoughts being taken out of your head?

-Did you ever feel as if your thoughts were being broadcast out loud so that other people could actually hear what you were thinking?

-Did you ever believe that someone could read your mind?

Have you ever experienced extreme beliefs that people or organizations are out to get you or that you are a very famous person?

If Yes: *All the same follow up questions as above*

Note: Not while under the influence of a substance (e.g., marijuana, LCD) or cultural experience (e.g., ancestor, spirits, god watching over, guardian angel). 
Have you ever had periods of a week or more in a row in which you were feeling 'up' or 'high' or 'hyper', like you were bouncing off the walls? Do you have periods when you feel so active or full of energy you get into trouble?

\section{If YES:}

During this time, did you feel like your thoughts were racing, you were full of ideas, and could do a lot things? Did you make impulsive decisions like spending a lot of money?

If YES: What was it like? (Was that more than just feeling good?) Did you also feel like you were "hyper" or "wired" and had an unusual amount of energy?

Were you much more active than is typical of you? (Did other people notice?)

If NO: Have you ever had a period of time when you were feeling irritable, angry, or short-tempered for most of the day, every day, for at least several days? What was that like? (Was that different from the way you usually are?)

-If YES: Did you also feel like you were "hyper" or "wired" and had an unusual amount of energy? Were you much more active than is typical of you? (Did other people comment on how much you were doing?) When was that? How long did that last? (As long as 1 week?)

IF LESS THAN ONE WEEK: Did you need to go into the hospital to protect you from hurting yourself or someone else, or from doing something that could have caused serious financial or legal problems? Did you feel (high/irritable/ OWN WORDS) for most of the day, nearly every day during this time? Have you had more than one time like that? (Which time was the most extreme?)

IF UNCLEAR: Have you had any times like that in the past year, since 1 YEAR AGO)?

During that time:

\section{Inflated self-esteem or grandiosity}

a. How did you feel about yourself? (More selfconfident than usual? Did you feel much smarter or better than everyone else? Did you feel like you had any special powers or abilities?

2. Decreased need for sleep (e.g., feels rested only after 3 hours of sleep)
No history of bipolar disorder. 
a. Did you need less sleep than usual? (How much sleep did you get?) If YES: Did you still feel rested?

3. More talkative than usual or pressure to keep talking?

a. Were you much more talkative than usual? (Did people have trouble stopping you or understanding you? Did people have trouble getting a word in edgewise?)

4. Flight of ideas or racing thoughts

a. Did you have thoughts racing through your head? (What was that like?)

5. Distractibility (i.e., attention too easily drawn to unimportant or irrelevant external stimuli) as reported or observed.

a. Were you so easily distracted by things around you that you had trouble concentrating or staying on one track? (Give an example)

6. Increase in goal-directed activity (either socially, at work or school, or sexually) or psychomotor agitation (i.e., purposeless non-goal-directed activity)

a. How did you spend your time? (Work, friends, hobbies? Were you especially busy during that time?)

b. (Did you find yourself more enthusiastic at work or working harder at your job? Did you find yourself more engaged in school activities or studying harder?)

c. (Were you more sociable during that time, such as calling on friends or going out more than you usually do or making a lot of new friends?)

d. (Were you spending more time thinking about sex or involved in doing something sexual, by yourself or with others? Was that a big change for you?)

e. Were you physically restless during this time, doing things like pacing a lot, or being unable to sit still? (How bad was it?)

7. Excessive involvement in activities which have a high potential for painful consequences (e.g., engaging in unrestrained buying sprees, sexual indiscretions, or foolish business investments)

a. During that time...did you do anything that could have caused trouble for you or your family? (Spending money on things you didn't need or couldn't afford? 


\begin{tabular}{|c|c|}
\hline $\begin{array}{l}\text { b. How about giving away money or valuable things? } \\
\text { (Gambling with money you couldn't afford to } \\
\text { lose?) } \\
\text { (Anything sexual that was likely to get you in trouble? Driving } \\
\text { recklessly?) (Did you make any risky or impulsive business } \\
\text { investments or get involved in a business scheme that you } \\
\text { wouldn't normally have done?) } \\
\text { Note: Not while under the influence of a substance (e.g., LSD) or } \\
\text { cultural experience (e.g., spirits, ancestor looking after). }\end{array}$ & \\
\hline $\begin{array}{l}\text { Do you have any developmental disabilities that would prevent you } \\
\text { from answering questions for a few hours, such as intellectual } \\
\text { disability, or an inability to comprehend or read sentences? }\end{array}$ & $\begin{array}{l}\text { No } \\
\text { neurodevelopmental } \\
\text { disorders. }\end{array}$ \\
\hline $\begin{array}{l}\text { Do you have any hearing impairments that would prevent you from } \\
\text { hearing sound or speech at a regular volume? }\end{array}$ & $\begin{array}{l}\text { No hearing } \\
\text { impairments }\end{array}$ \\
\hline Are you color-blind? & Not color-blind \\
\hline $\begin{array}{l}\text { Do you have any visual impairment that is not corrected by glasses or } \\
\text { contacts? }\end{array}$ & No vision impairments \\
\hline $\begin{array}{l}\text { Have you ever suffered a head injury that caused you to lose } \\
\text { consciousness or that resulted in disorientation and confusion, loss of } \\
\text { memory, dizziness? } \\
\text { If YES: How long were you unconscious? } \\
\text { (> } \mathbf{3 0} \text { minutes unconscious = Moderate to Severe TBI) }\end{array}$ & $\begin{array}{l}\text { No moderate to severe } \\
\text { TBIs }\end{array}$ \\
\hline $\begin{array}{l}\text { Are you currently pregnant? } \\
\text { Do you have any medical condition (e.g., heart condition) that could } \\
\text { be affected by receiving a mild shock? }\end{array}$ & $\begin{array}{l}\text { No medical conditions } \\
\text { exacerbated by shock. }\end{array}$ \\
\hline $\begin{array}{l}\text { Do you have a romantic partner, like a girl/boyfriend, spouse, etc.? } \\
\text { If YES: Would you allow us to contact this person and } \\
\text { another person that knows you well such as a close friend, } \\
\text { roommate, or family member to ask them questions about you } \\
\text { as part of the study? (e.g., about personality, behavior). They } \\
\text { would be compensated \$15 for their participation. } \\
\text { If NO: Do you have } 1 \text { or } 2 \text { persons that know you well such } \\
\text { as a close friend, roommate, or family member that you would } \\
\text { allow us to contact to ask them questions about you as part of } \\
\text { the study? They will be compensated } \$ 15 \text { for their } \\
\text { participation. }\end{array}$ & $\begin{array}{l}\text { Two informants, but } \\
\text { one is acceptable. }\end{array}$ \\
\hline
\end{tabular}


Since the sensors in the cap need to make direct contact with the scalp to be able to measure brain activity, I need to ask you whether your hair is worn quite big (e.g., large afros or dreadlocks, or thick braids that do not allow the cap to reach the scalp), is that the case for you?

If YES (or maybe like a smaller afro/larger head): "I do need to make you aware of the possibility that we might not be able to do the study if unable to apply the cap; we will do our best to do so; that being said, we want to inform you of this possibility in advance."
No large afros, cornrows, large braids, or dreadlocks; not sewn on weaves or glued hair extensions.

"Okay, I'm going to enter your responses into the computer and it will tell me whether you qualify for the study. This will take a few seconds, please hold."

Put the person on hold and review the screening answers to make sure they qualify/don't qualify.

If the person DOES NOT QUALIFY: "Unfortunately, you do not qualify to be a participant on this study. Thank you so much for taking the time to talk to me today!"

If the person wants to know WHY they don't qualify: "I'm not permitted to disclose this information. The study requires anyone participating to meet a specific set of criteria, and unfortunately, your answers indicate you do not meet one or more of these criteria. Thank you so much for taking the time to talk to me today!"

\section{If the person DOES QUALIFY:}

"Congratulations! You are eligible to participate in our study! Next I will be explaining a bit more about how the study works and what you can expect from your study visit so that you can decide if you want to make an appointment. But first, let me take down basic contact information from you."

\section{Full Name:}

\section{Primary Phone Number:}

"Is this the most reliable phone number to reach you at for a reminder call the day before your study visit?” YES NO

"Is it okay if we leave you a voicemail at this number?" YES NO

"Is it okay if we send reminder texts to this number?" $\quad$ YES NO

\section{Secondary Phone Number:}

\section{Email Address:}

"If you agree to participate, we will send you a follow-up email with the date and time of your appointment as well as directions to our lab." 
"This study consists of two sessions taking place in separate days within the same week, if possible, with both scheduled today should you decide to participate. In these sessions, you will be asked to complete questionnaires, interviews, and computer tasks during which your brain activity will be monitor with sensors placed on your head and face. During one of the computer tasks you will experience minor shocks; these are neither painful nor dangerous and if you feel discomfort at any point, you can end your participation.

You will earn $\$ 40$ for each session in which you participate. In addition, you will be eligible to $\$ 10$ bonus for each session you attend the first time scheduled and an additional $\$ 10$ bonus for completing both sessions in a one week. In total, you can earn up to \$110. You will be also given the opportunity to participate in another paid study. Do you have any questions?"

Are you still interested in making appointments?" YES NO

"Can you make Session 1 on either Monday or Tuesday and Session 2 on either Thursday or Friday of the same week?”

\section{Session 1 Appointment Date/Time:}

\section{Session 2 Appointment Date/Time:}

"Okay, I have scheduled you for Session 1 on [day/ time] and for Session 2 on [day/time]"

"For your study visit, you will be coming to the Psychology Building at the University of South Florida, Tampa Campus. The physical location of our lab within campus is 3711 USF Citrus Drive. Free parking will be provided when you arrive. You can park in any spot labeled CSD/PSY. The study will take place in room 2110 (on the second floor)."

\section{Would you be needing a parking pass? YES NO}

"We understand this is a lot of information to remember, we will be including directions to our lab in the confirmation email sent to you immediately after this phone call. We will also send you a brief reminder via text message. If you have any difficulty finding, the lab feel free to call us and ask for directions! If you need to cancel or reschedule your appointment, please notify us within 24 hours of your session In addition, if you are running late on the day of your study session, please give us a call as well to let us know. Remember that if you make it to your first appointment as scheduled today, you will be automatically eligible for a $\$ 10$ bonus. Do you have any questions? Okay, if you have any questions between now and the day of your session, or if you need to reschedule your session, please calls us at (813) 974 - 4393.'

\section{Post-Phone Screen Checklist}

Confirmation email sent to participant.

Appointment date/time entered added to Scheduling Google Calendar. Appointment information added to the Appointment Tracker. Email RA and Grad Students notifying them of the session. Double-Check that they have not previously participated or disqualified 


\section{APPENDIX D. Informed Consent}

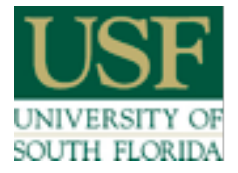

\section{Informed Consent to Participate in Research Involving Minimal Risk}

Pro \# 00027233

You are being asked to take part in a research study. Research studies include only people who choose to take part. This document is called an informed consent form. Please read this information carefully and take your time making your decision. Ask the researcher or study staff to discuss this consent form with you, and please ask him/her to explain any words or information you do not clearly understand. The nature of the study, risks, inconveniences, discomforts, and other important information about the study are listed below.

We are asking you to take part in a research study called: Transdiagnostic Affective \& Cognitive Systems (TACoS, for short)

The person who is in charge of this research study is Dr. Edelyn Verona at the University of South Florida. This person is called the Principal Investigator. However, other research staff may be involved and can act on behalf of the person in charge.

The research will be conducted at the University of South Florida in Tampa, FL.

This research is being sponsored by the National Institute on Mental Health (NIMH).

\section{Purpose of the study}

The purpose of the study is to examine the effects of emotions on decision-making and interpersonal judgments. This research question will be carried out through a series of questionnaires and a short interview, as well as a decision-making task and an interpersonal judgment task.

\section{Why are you being asked to take part?}

We are asking you to take part in this research study because we are examining how persons respond to a series of tasks related to emotion and decision-making. You are an undergraduate student at USF and fluent in English. 


\section{Study Procedures:}

If you take part in this study, you will complete one or two sessions (depending on the phase of the experiment you are in).

In the first session, the experiment involves questionnaires, interviews, and/or a computer task (you may be asked to complete only some or all of these). This session will take about 3 hours of your time. First, you may be asked to complete several questionnaires and a short interview about your emotions, your behaviors, your relationships with others, and how you handle stress. Some questions will ask about sensitive information (e.g., aggression, substance use, sexual behavior, and violence). We will be recording the interviews using a digital audio recorder if you give us permission to do so. Audio recording is optional. You can skip any questions on the questionnaires that you may not wish to answer. You will also be asked to complete a paper screening measure to ensure you have not used any drugs or alcohol (within 24 hours), as this may affect your responses and performance in our study.

Second, you may be asked to provide contact information for at least 1 close friend/relative or romantic partner. Study personnel will contact the individual to ask them questions about your recent behaviors. Allowing us to contact your friends/relatives/significant others will improve our way of measuring your emotions and behaviors.

Third, you may complete a decision-making task while we record your brain waves. In this task, you will be asked to make decisions about the letters, shapes or numbers presented to you. During these tasks, an electrode net with sensors attached to it will be placed on your head. You will also have sensors attached around your nose and eye area; we will use an alcohol pad and exfoliating cream to prepare the skin before attaching the sensors. These sensors are generally nonirritating because the paste used to connect them has a salt concentration similar to that of human perspiration. The electrodes are also not invasive, do not produce any long-term marks or scarring, and should not cause any long-term discomfort. If the sensors attached to the face need to be applied twice due to a bad connection, we will ensure that you are not experiencing too much discomfort. You will sit in front of a computer while letters, numbers, or symbols will be shown to you. We will be using a webcam on the computer to passively monitor you during the experiment. This will be used for observation only; no video recordings will be made.

For this task, you will also be wearing earphones/headphones. Sudden, brief (1/20th of a second) bursts of white noise (not exceeding $105 \mathrm{~dB}$, about the volume of a subway train, for a fraction of a second) will be heard at various points throughout the study. These noises, which sound like loud static, might be experienced as startling, but they are neither painful nor harmful. During this task, you will also experience minor shocks administered through electrodes attached to your two fingers. These shocks feel like the "zap" you may feel when touching a door knob. You will experience these several times. Should you experience major discomfort from these shocks, and wish to discontinue participation, you may do so at any time. Other persons who have participated in this study have described the shocks as unpleasant but not painful, and they are not harmful in any way. Safeguards have been made to assure your complete safety during these procedures.

If you are asked to come in for the second session, it may include another decision-making task on the computer and/or an interpersonal judgment task. This session takes approximately 2 hours 
to complete. The computer task will ask you to respond as quickly as you can to certain words but not others. Just like in the session 1 computer task, you will wear an electrode net with sensors attached to it and sit in front of a computer while words are shown to you. In the interpersonal judgment task, you and another participant may rotate roles of being an employee and supervisor. To measure how we evaluate others, you may be asked to write an essay about yourself and your qualities for review by the other participant. Or you may be asked to review the essay written by a different participant. Then, you will perform a supervisor-employee task. During this task, you may serve as the employee or supervisor, in which you will provide feedback via shocks when the employee makes an incorrect response (if you are the supervisor), or you will receive feedback about your incorrect responses (if you are the employee).

\section{Total Number of Participants}

About 300 participants will take part in this study at USF and approximately 400 close friends, relatives or romantic partners will be asked to complete some questionnaires about them. A total of 700 individuals will participate in the study.

\section{Alternatives / Voluntary Participation / Withdrawal}

You have the alternative to choose not to participate in this research study or to do other SONA research studies for SONA credit.

You should only take part in this study if you want to volunteer. You should not feel that there is any pressure to take part in the study. You are free to participate in this research or withdraw at any time. There will be no penalty or loss of benefits you are entitled to receive if you stop taking part in this study. Whether or not you take part will not affect your student status or your relationship with USF.

\section{Benefits}

We are unsure if you will receive any direct benefits by taking part in this research study, except helping us improve our understanding of emotions and decision-making.

\section{Risks or Discomfort}

The following risks may occur:

- You may experience some discomfort answering questions about your emotions, behaviors and distressing experiences. If you experience any distress due to any part of the study, you may contact the University of South Florida Psychological Services Center at 813-974-2496. You may also call the Crisis Center of Tampa Bay at 813-964-1964 or the National Suicide Prevention Lifeline at 1-800-273-TALK (8255), both of which are available 24 hours a day, 7 days a week. In addition, a resource sheet with community and campus resources will be provided at the end of this study - or upon request at any time (even if you choose not to participate).

- You may experience some physical discomfort associated with application of the brain data collection equipment (EEG cap), and electrodes to your face, exposure to electric shocks, or exposure to bursts of noise. There is no reason to believe that any discomfort or pain you may experience will exceed that normally encountered in everyday life (e.g., 
getting zapped when touching a door knob, listening to loud music). Any discomfort you may experience should occur for only a short period of time. It is very unlikely that you will experience lasting physical harm from your participation in these aspects of the study. We will also check with you throughout the session to ensure that you are not experience too much discomfort.

- You may be concerned about disclosing private information to study staff. To protect your privacy, we will assign you a number to be used in place of your name on all materials. No one will be given information about you or your responses. All data will be stored under lock and key. To further help us protect your privacy, we have obtained a Certificate of Confidentiality from the National Institutes of Health. This certificate allows us to resist any attempts by lawyers or judges to identify you. We cannot be forced to give over any information or data about you, even by a court subpoena. You can still choose to release information about yourself if you want to do that. If an insurer, employer or other person obtains written consent from you to receive research information, then we will provide that information to them.

- The only time we would have to tell someone about what you say is if a child, elderly or disabled person is being abused or you are going to hurt yourself or others. We will only inform someone if there is immediate danger to you or another person.

\section{Compensation}

Session 1 takes about 3 hours to complete, and you will get 1 SONA credits for every 30 minutes you take part in the study = up to 6 SONA credits. Session 2 takes 2 hours, and you will get 1 SONA credits for every 30 minutes you take part in the study $=$ up to 4 SONA credits.

\section{Costs}

It will not cost you anything to take part in the study.

\section{Privacy and Confidentiality}

We will keep your study records private and confidential. Besides the research team, a few other people may need to see your study records, although most of the time no one else will see your records. Anyone who looks at your records must keep them confidential. These individuals include:

- The research team, including the Principal Investigator, study coordinator, and all other research staff.

- Certain government and university people who need to know more about the study, and individuals who provide oversight to ensure that we are doing the study in the right way.

- Any agency of the federal, state, or local government that regulates this research, including the Office for Human Research Protection.

- The USF Institutional Review Board (IRB) and related staff who have oversight responsibilities for this study, including staff in USF Research Integrity and Compliance.

- The funders of this study have asked that we share deidentified data (without names or identifiers) from this study to the National Institute of Mental Health Data Archive (NDA), a large database run by the National Institute of Mental Health (NIMH). If you agree to have your data shared, all of your personal information such as name, address, and phone number will be removed and replaced with a code number. The information 
provided to NDA may help researchers find out more about how to treat problems with emotions and behaviors, including mental illness. You may decide now or later that you do not want to share your information using NDA. If so, contact the researchers who conducted this study, and they will tell NDA to stop sharing your research information. However, NDA cannot take back information that was shared before you changed your mind. If you would like more information about NDA, this is available on-line at http://data-archive.nimh.gov.

We may publish what we learn from this study. If we do, we will not include your name other information. We will not publish anything that would let people know who you are.

\section{You can get the answers to your questions, concerns, or complaints}

If you have any questions, concerns or complaints about this study, or experience an unanticipated problem, call Dr. Edelyn Verona at (813) 974-0392.

If you have questions about your rights as a participant in this study, or have complaints, concerns or issues you want to discuss with someone outside the research, call the USF IRB at (813) 974-5638 or contact by email at RSCH-IRB@usf.edu.

\section{Consent to Take Part in this Research Study}

I freely give my consent to take part in this study. I understand that by signing this form I am agreeing to take part in research and that I am 18 years of age or older. I have received a copy of this form to take with me.

CONSENT TO AUDIO-RECORD

Yes, my interview may be audio-recorded.

No, my interview may not be audio-recorded.

CONSENT TO SHARE YOUR DE-IDENTIFIED DATA WITH NDA

Yes, my information (without any identifiers) can be shared with NDA.

No, my information cannot be shared with NDA.

CONSENT TO BE CONTACTED FOR FUTURE STUDIES

Yes, please contact me about future study participation opportunities.

No, you may not contact me about future study participation opportunities.

Signature of Person Taking Part in Study

Date

Printed Name of Person Taking Part in Study 


\section{Statement of Person Obtaining Informed Consent}

I have carefully explained to the person taking part in the study what he or she can expect from their participation. I confirm that this research subject speaks the language that was used to explain this research and is receiving an informed consent form in their primary language. This research subject has provided legally effective informed consent.

Signature of Person obtaining Informed Consent

Date

Printed Name of Person Obtaining Informed Consent

Date 


\section{APPENDIX E. \\ Participant Demographics Response Form}

Please fill out or circle the following information about yourself. All responses are completely confidential.

1. Zip Code:

2. Age:

3. Sex:

1. Female

2. Male

3. Transgender (male to female)

4. Transgender (female to male)

5. Other (please describe)

\section{Ethnicity:}

1. Hispanic

2. Not-Hispanic

5. Race:
1. Caucasian (White)
2. African American (Black)
3. Asian descent
4. Latino/a
6. Native American
7. Mixed ethnicity
8. Middle Eastern/North African
5. Pacific Islander or Hawaiian
9. Other:

6. Household income (if you are a full-time student or dependent, select your parent/guardian household income):
1. less than $\$ 15,000$
4. $\$ 45,001-60,000$
2. $\$ 15,000-30,000$
5. $\$ 60,001-75,000$
3. $\$ 30,001-45,000$
6. Over $\$ 75,000$

\section{What is your occupation (job)?}

1. Service worker or laborer, for example maid, bellhop, janitor, stock handler, farm laborer, car washer, entry-level factory work, unemployed for long periods of time

2. Untrained worker, for example restaurant help (busboy, waiter/waitress), bartender, cook, waste management (garbage collector), gardener, parking attendants

3. Machine operators and semi-skilled worker, for example machine operator/ excavation, painter, barber, bus driver, chauffeur, child care worker, hairstylist/beautician, health or nurse aide/assistant, butcher, roofer, taxicab driver, truck driver, non-commissioned soldier, housekeeper

4. Skilled manual worker, for example tenant farmers, small business owner, flight attendant, plumber, carpenter, decorator detective, drywall/carpet installer, 
electrician, firefighter, machinist, mail carrier, mechanic, police/law enforcement, receptionist, tailor, welder, jeweler, meter reader, repairmen

5. Clerical and sales worker, for example secretary, bank teller, bookkeeper, recreation worker, library attendant, bill account collector

6. Technician and semi-professional, for example medium-size farm owner, advertising agent, dental hygienist, legal secretary, foremen, photographer, sheriff, occupational therapist, construction inspector, air traffic controller

7. Manager and other professional, for example actor or entertainer, computer programmer, funeral director, office/sales manager (not retail), public relations, insurance adjustor, realtor, reporter, social worker, elementary or middle school teacher, vocational counselor

8. Administrator and technical professional, for example district manager of large business, accountant, professional clergy, chiropractor, pharmacist, registered nurse, high school principal or high school teacher, computer analyst, airplane pilot, author /editor

9. Executive and major professional, for example the chairperson, (vice) president, owner or treasurer of large business, corporation, or farm; lawyer, judge, doctor, college professor, engineer, architect dentist, commissioned officer (major, lieutenant, commander

10. Homemaker

11. Unemployed

12. Other (specify) (e.g., retired)

\section{How did you hear about our study?}

1. Flyer in community (coffee shop, supermarket, convenience store)

2. Newspaper Ad

3. Craigslist Ad

4. Other Online Ad (e.g., Creative Loafing, Reddit)

5. Ad posted on public transportation (e.g. bus stop, on a bus, etc.)

6. Probation/ Parole or court house

7. SONA subject pool

8. Contacted via mail, email, and/or phone from our research team

9. Participated in previous research study

10. Heard about it from a friend or relative

11. Who told you about the study (e.g. friend, sister, etc.)?

12. Other (Please describe):

9. Are you right or left handed?
1. Right

2. Left

10. Are you currently taking any medications for a psychological condition (for example, depression, schizophrenia, anxiety)?

1. Yes

1. No

If yes, what are the medications and/or what are they for?

11. In the last 48 hours, have you used any types of drugs (e.g., marijuana, cocaine, heroin, meth, pain pills)

1. Yes 
1. No

If yes, which drugs did you take?

12. In the last 48 hours, have you drank any alcohol?

2. Yes

1. No

If yes, how much did you drink (if you drank more than one type of drink, please indicate how much you drank of each type)?

13. Do you smoke (i.e., cigarettes, e-cigarettes)?

2. Yes

1. No

If yes:

What do you smoke (please circle)? Cigarette E-Cigarettes Both

How frequently do you smoke?

How much do you smoke each day (e.g., one pack)?

When was the last time you smoked?

Will you need smoke breaks during the study?

1. Yes

1. No 


\section{APPENDIX F. \\ Shock Sensitivity Evaluation Form}

\begin{tabular}{|c|c|c|}
\hline \multicolumn{3}{|c|}{$\begin{array}{l}\text { Use the shock LEVEL that corresponds to the shock that the participant rates as } 50 \text { in Eprim } \\
\text { Startup info for NPU Part } 2\end{array}$} \\
\hline Shock \# & $\begin{array}{l}\text { Participant's Shock Rating }(0- \\
100)\end{array}$ & $\begin{array}{l}\text { Shock Level (use this in startup info for } \\
\text { part 2) }\end{array}$ \\
\hline 1 & & 3 \\
\hline 2 & & 15 \\
\hline 3 & & 27 \\
\hline 4 & & 39 \\
\hline 5 & & 51 \\
\hline 6 & & 63 \\
\hline 7 & & 75 \\
\hline 8 & & 87 \\
\hline 9 & & 99 \\
\hline 10 & & 111 \\
\hline 11 & & 123 \\
\hline 12 & & 135 \\
\hline 13 & & 147 \\
\hline 14 & & 159 \\
\hline 15 & & 171 \\
\hline 16 & & 183 \\
\hline 17 & & 195 \\
\hline 18 & & 207 \\
\hline
\end{tabular}




\begin{tabular}{|c|c|c|}
\hline 19 & $\mathbf{2 1 9}$ \\
\hline 20 & $\mathbf{2 3 1}$ \\
\hline 21 & & $\mathbf{2 4 3}$ \\
\hline 22 & $\mathbf{2 5 4}$ \\
\hline
\end{tabular}




\section{APPENDIX G. UPPS-P Impulsive Behavior Scale}

Instructions: Below are a number of statements that describe ways in which people act and think. For each statement indicate how much you agree or disagree. Answer based upon your own experiences and beliefs rather than those of other people. Use the scale to find the answer that best represents how you generally tend to feel or act.

1

$\begin{array}{ll}4 \\ \text { Disagree strongly } & \text { Disagree somewhat }\end{array}$
3

Agree somewhat Agree strongly

1. I have a reserved and cautious attitude towards life

2. I have trouble controlling my impulses

3. I generally seek new and exciting experiences and sensations

4. I generally like to see things through to the end

5. When I am very happy, I can't seem to stop myself from doing things that can have bad consequences

6. My thinking is usually careful and purposeful

7. I have trouble resisting my cravings (for food, cigarettes, etc.)

8. I'll try anything once

9. I tend to give up easily

10. When I am in great mood, I tend to get into situations that could cause me problems

11. I am not one of those people who blurt out things without thinking.

12. I often get involved in things I later wish I could get out of

13. I like sports and games in which you have to choose your next move very quickly

14. Unfinished tasks really bother me

15. When I am very happy, I tend to do things that may cause problems in my life

16. I like to stop and think things over before I do them.

17. When I feel bad, I will often do things I later regret in order to make myself feel better now

18. I would enjoy water skiing

19. Once I get going on something I hate to stop

20. I tend to lose control when I am in a great mood

21. I don't like to start a project until I know exactly how to proceed

22. Sometimes when I feel bad, I can't seem to stop what I am doing even though it is making me feel worse

23. I quite enjoy taking risks

24. I concentrate easily

25 . When I am really ecstatic, I tend to get out of control 
26. I would enjoy parachute jumping

27. I finish what I start

28. I tend to value and follow a rational "sensible" approach to things

29. When I am upset I often act without thinking

30. Others would say I make bad choices when I am extremely happy about something.

31. I welcome new and exciting experiences and sensations, even if they are a little frightening and unconventional

32. I am able to pace myself so as to get things done on time

33. I usually make up my mind through careful reasoning

34. When I feel rejected, I will often say things that I later regret

35. Others are shocked or worried about the things I do when I am feeling very excited

36. I would like to learn to fly an airplane

37. I am a person who always gets the job done

38. I am a cautious person

39. It is hard for me to resist acting on my feelings

40. When I get really happy about something, I tend to do things that can have bad consequences

41. I sometimes like doing things that are a bit frightening

42. I almost always finish projects that I start

43. Before I get into a new situation I like to find out what to expect from it

44. I often make matters worse because I act without thinking when I am upset

45. When overjoyed, I feel like I can't stop myself from going overboard.

46. I would enjoy the sensation of skiing very fast down a high mountain slope

47. Sometimes there are so many little things to be done that I just ignore them all

48. I usually think carefully before doing anything

49. When I am really excited, I tend not to think of the consequences of my actions

50. In the heat of an argument, I will often say things that I later regret

51. I would like to go scuba diving

52. I tend to act without thinking when I am really excited

53. I always keep my feelings under control

54. When I am really happy, I often find myself in situations that I normally wouldn't be comfortable with

55. Before making up my mind, I consider all the advantages and disadvantages

56. I would enjoy fast driving

57. When I am very happy, I feel like it is ok to give in to cravings or overindulge

58. Sometimes I do impulsive things that I later regret

59. I am surprised at the things I do while in a great mood. 


\section{APPENDIX H. \\ Lifetime History of Aggression (LHA) Interview}

Conduct a "semi-structured" interview so that the following items may be rated. Please note that only reported actual behavior (e.g. verbal and/or physical) can be rated in the assessment of an item category. Aggressive thoughts, attitudes, and fantasies are not counted. It is important to rate any events that have occurred over the subject's lifetime (including years as a teenager and young adult). Please take as many notes as possible but score only behaviors from age $\mathbf{1 3}$ onward.

$$
\begin{aligned}
& 0=\text { no events } \\
& 1=\text { one event } \\
& 2=\text { "a couple" or "a few" (i.e., 2-3) events } \\
& 3=\text { "several" or "some" (i.e., 4-9) events } \\
& 4=\text { "many" or "numerous" (i.e., 10+) events } \\
& 5=\text { "so many events that they can't be counted" }
\end{aligned}
$$

Item Categories / Item Questions:

1. Temper tantrums that are developmentally inappropriate (i.e. behavioral manifestations in response to frustration; screaming, ranting and raving, throwing things, etc.).

Do you ever get in any temper tantrums when you are frustrated, specifically ranting and raving, stomping around, screaming?

If NO ---> Since the age of 13 , have you ever gotten in any temper tantrums (e.g., ranting and raving, stomping around, screaming)?

\section{How many times?}

2. Physical fighting (e.g. history of physical fights with other people whether or not the subject started the fight or not, come to blows with other people).

\section{How many physical fights have you been in since you turned 13?}

3. Verbal fighting (e.g. history of verbal arguments in which an angry voice / profanity / insults / threats are used. Individual being assessed needs to be the one who is verbally aggressive in altercations, whether or not the other person also is verbally aggressive. Polite disagreements and/or very minor altercations are not to be scored as positive). 
How many verbal fights have you been in since you turned 13? (e.g. angry voice / profanity / insults / threats are used).

4. Specific assaults on other people NOT during a physical fight (jumping, assaulting, and/or attacking another person without provocation; hurting someone for fun).

Since the age of 13, have you ever assaulted another person, NOT in a physical fight, like jumping them or hurting them for fun, or to get back at them for insulting you in the past? How many times? Who and why?

If unsure whether person engaged in behavior without provocation, ask:

Was there a verbal altercation prior to this happening?

5. Specific assaults on property (i.e., hitting / throwing / breaking objects, windows, dishes, etc.; count all behaviors that also occur in the context of a verbal fight or temper tantrum).

Since the age of 13, have you ever done any vandalism? What was it? How about destroyed property to get back at someone or because you were angry; how about because you thought it was fun to destroy someone else's stuff (e.g., break windows, throw dishes, destroy property, punch wall)? How many times?

6a. Specific assaults on self (i.e., self-injurious, but not suicidal, in nature; do NOT include tattooing and/or piercing).

Since the age of 13, have you ever injured yourself on purpose (e.g., burning, cutting) with no intention to kill yourself, like cutting or burning or punching yourself? How many times?

6b. Suicide attempts.

Since the age of 13, have you ever attempted suicide (with the intention to kill yourself)?

If NO ----> Since the age of 13 , have you ever hurt yourself with at least some intention of killing yourself?

How many times?

7. School disciplinary problems (e.g. reprimand by school principal, suspension, expulsion; score only those that occurred after the age of 13).

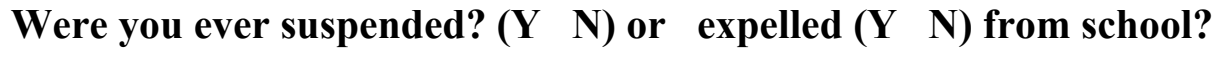
1. Ages:
1. Ages:
Total S:

2. Reasons:

2. Reasons:

E: 
8. Problems with supervisors at work (e.g. behavioral outbursts in response to authority, reprimands, demotions, or terminations due to aggressive/impulsive behaviors).

How did you get along with your supervisors? Did you ever have behavioral outbursts in response to authority (e.g., verbal altercations, talking back to supervisor resulting in reprimands)? What about reprimands, demotions, or terminations due to aggressive/impulsive behaviors?

How many times has this happened since the age of 13 (i.e., count number of separate events)?

9. Antisocial behavior not involving the police (e.g. lying, stealing, selling drugs, involvement in illegal operations, violations of the rights of others).

Since the age of 13, have you done things that are illegal or would be grounds to get arrested, without getting caught by the police (e.g., shoplifting, driving under the influence, conning others, selling drugs, or committing a felony, sexual offenses)?

How many times?

Everyone tells a few lies, but have you told a lot of lies to obtain goods/favors or to avoid obligations (to get out of trouble)? Have you ever used an alias? Have these things happened since the age of 13 ? How many times?

Since the age of 13, have you ever stolen anything without getting caught by the police? How many times?

Since the age of 13, have you taken advantage of another person, or conned them? How often has this happened?

How many times have you driven intoxicated from drinking or using marijuana or other drugs?

10. Antisocial behavior involving the police (e.g. warnings, arrests and/or convictions for misdemeanor or felony offenses).

Have you ever gotten in trouble with the police for your behaviors, this may include drinking and driving, reckless driving, public intoxication, causing a riot, etc., even if they did not result in any charges or convictions?

What did you do?

How many times?

How old were you the first time you were ever arrested?

What was it for? Is there anything on your juvenile record?

What about your adult record? 
What about any other arrests that did not result in charges and/or convictions?

How many times?

Total Score:

(0-55) 
I1.

\section{APPENDIX I. \\ MINI - I. Alcohol Use Disorder (AUD)}

In the past 12 months, have you drank alcohol?

NO

YES

If YES, ask -

How many times per month do you drink alcohol?

How many alcoholic drinks per occasion do you drink?

(alcoholic drink reference provides guidance on what constitutes 1 alcoholic drink)

Before the past 12 months, has there ever been another period when you were drinking more?

NO

YES

If YES, ask -

When was this period?

How long did this period last?

During this period, how many times per month were you drinking?

During this period, how many alcoholic drinks per occasion were you drinking?

I2.

\begin{tabular}{|c|c|c|c|c|}
\hline \multirow[b]{2}{*}{$\begin{array}{l}\text { a. During the times when you drank alcohol, did you end } \\
\text { up drinking more than you planned when you started? }\end{array}$} & \multicolumn{2}{|c|}{$\begin{array}{l}\text { In the past } 12 \\
\text { months: }\end{array}$} & \multicolumn{2}{|c|}{ Lifetime: } \\
\hline & NO & YES & NO & YES \\
\hline $\begin{array}{l}\text { b. Did you repeatedly want to reduce or control your } \\
\text { alcohol use? } \\
\text { Did you try to cut down or control your alcohol use, but } \\
\text { failed? } \\
\text { IF YES TO EITHER, CODE YES. }\end{array}$ & NO & YES & NO & YES \\
\hline $\begin{array}{l}\text { c. On the days that you drank, did you spend substantial } \\
\text { time obtaining alcohol, drinking, or recovering from the } \\
\text { effects of alcohol? }\end{array}$ & NO & YES & NO & YES \\
\hline $\begin{array}{l}\text { d. Did you crave or have a strong desire or urge to use } \\
\text { alcohol? }\end{array}$ & NO & YES & NO & YES \\
\hline e. Have/did you missed work or school or often arrive late & NO & YES & NO & YES \\
\hline
\end{tabular}


(or do a bad job) because you were intoxicated/drunk or hungover? Did you stop meeting obligations at home (e.g., taking care of kids)?

f. Did your drinking caused problems with your family or other people? If so, did you still keep on drinking?

g. Were you intoxicated more than once in any situation where you or others were physically at risk, for example, driving a car, riding a motorcycle, using machinery, boating, etc.?

h. Did you continue to use alcohol, even though it was clear that the alcohol had caused or worsened psychological or physical problems?

i. Did you reduce or give up important work, social or recreational activities because of your drinking?

j. Did you need to drink a lot more in order to get the same effect that you got when you first started drinking, or did you get much less effect with continued use of the same amount?

K SUMMARY: IF YES TO k1 OR k2, CODE YES

k1. When you cut down on heavy or prolonged drinking did you have any of the following (check if endorsed):

1. increased sweating or increased heart rate,

2. hand tremor or "the shakes"

3. trouble sleeping

4. nausea or vomiting

5. hearing or seeing things other people could not see or hear or having sensations in your skin for no apparent reason

6. Agitation

7. Anxiety

8. Seizures

IF YES TO 2 OR MORE OF THE ABOVE 8, CODE k1 AS YES.

NO YES NO YES

NO YES NO YES

NO YES NO YES

NO YES NO YES

NO YES NO YES

NO YES NO YES

k2. Did you drink alcohol to reduce or avoid withdrawal symptoms or to avoid being hungover?

NO YES NO YES 


\section{PAST 12 MONTHS:}

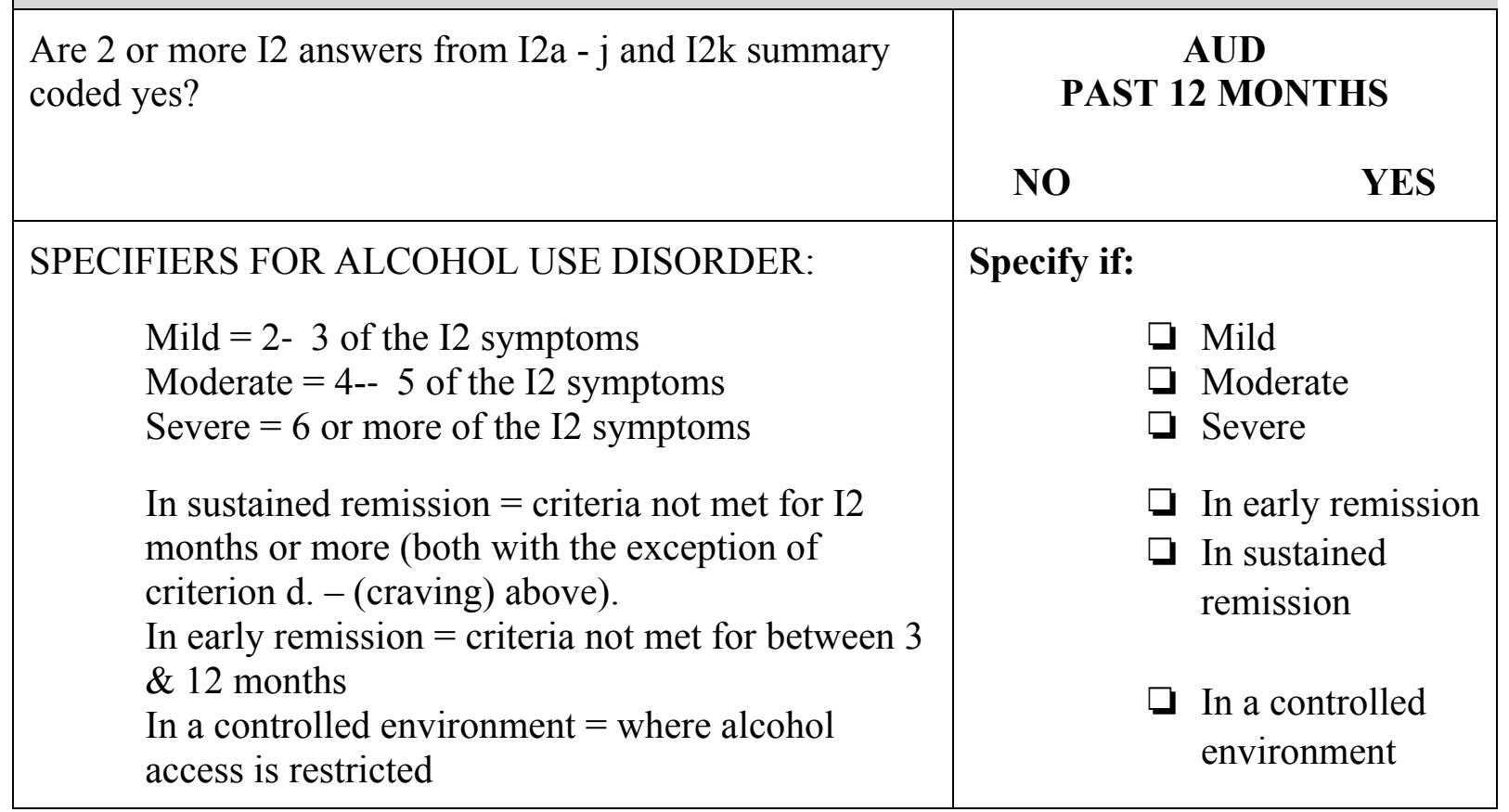

\section{LIFETIME}

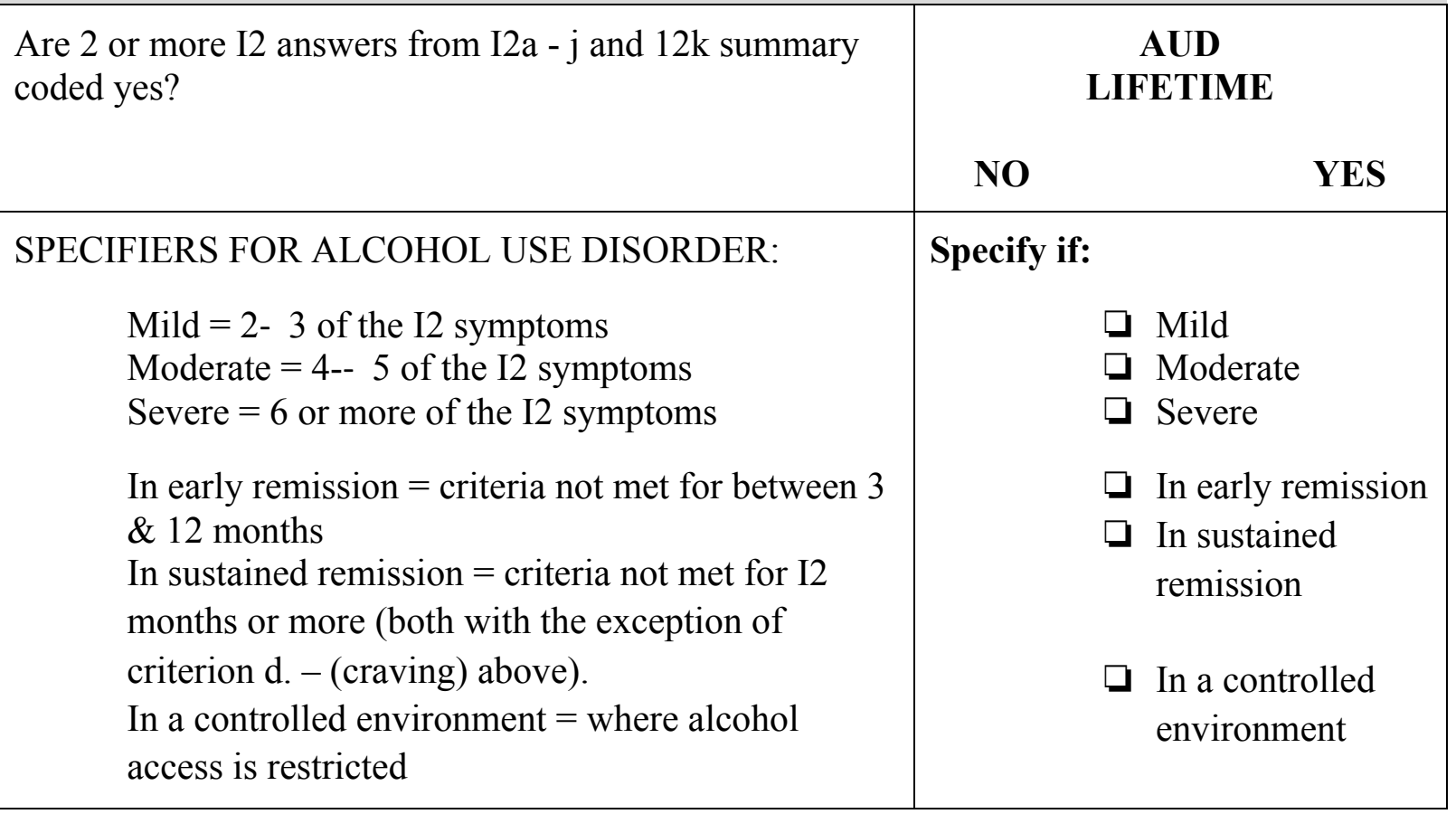




\section{APPENDIX J. \\ MINI - J. Substance Use Disorder (SUD) (non-alcohol)}

J1.

In the past 12 months, have you taken any of these drugs, to get high, to feel elated, to get "a buzz" or to change your mood?

If YES, in the past 12 months -

Which drug have you used the most?

Which drug have you used for the longest period of time?

Which drug has caused you the most problems?

Before the past 12 months, have you taken any of these drugs, to get high, to feel elated, to get "a buzz" or to change your mood?

If YES, in the past 12 months -

Which drug have you used the most?

Which drug have you used for the longest period of time?

Which drug has caused you the most problems?

Next to each drug category endorsed, indicate:

Circle each drug taken (only if outside prescribed amount)

Level of use \& number of times used in last month

Level of use for the period of heaviest use in lifetime.

\begin{tabular}{|c|lll|l|}
\hline $\begin{array}{c}\text { Number of times } \\
\text { used in last month }\end{array}$ & $\begin{array}{l}\text { Level of use } \\
\text { (circle one) }\end{array}$ & \multicolumn{1}{|c|}{ Drug taken } \\
\hline & 1 & 2 & 3 & $\begin{array}{l}\text { Stimulants: amphetamines, "speed", crystal meth, } \\
\text { "crank", dexedrine, ritalin, diet pills, bath salts, "black } \\
\text { beauties" }\end{array}$ \\
\hline & 1 & 2 & 3 & $\begin{array}{l}\text { Cocaine: snorting, "white girl", iv, freebase, crack, } \\
\text { "speedball" }\end{array}$ \\
\hline & 1 & 2 & 3 & $\begin{array}{l}\text { Opiates: heroin, morphine, dilaudid, opium, demerol, } \\
\text { methadone, darvon, codeine, percodan, vicodin, } \\
\text { oxycontin, fentanyl }\end{array}$ \\
\hline
\end{tabular}




\begin{tabular}{|c|c|c|c|c|}
\hline & 1 & 2 & 3 & $\begin{array}{l}\text { Hallucinogens: LSD ("acid"), mescaline, peyote, } \\
\text { psilocybin, STP, "mushrooms", "ecstasy", MDA, } \\
\text { MDMA ("molly"). }\end{array}$ \\
\hline & 1 & 2 & 3 & $\begin{array}{l}\text { Dissociative drugs: PCP (phencyclidine, "angel dust", } \\
\text { "peace pill", "hog"), or Ketamine ("Special K"). }\end{array}$ \\
\hline & 1 & 2 & 3 & $\begin{array}{l}\text { Inhalants: "glue", paint, ethyl chloride, "rush", nitrous } \\
\text { oxide ("laughing gas", "whippets"), amyl or butyl } \\
\text { nitrate ("poppers"). }\end{array}$ \\
\hline & 1 & 2 & 3 & $\begin{array}{l}\text { Cannabis: marijuana, hashish ("hash”), THC, "pot”, } \\
\text { "grass", "weed", "reefer", "dabs", "wax", "oil", "bud", } \\
\text { "green", "ganja", "kush." Spice. }\end{array}$ \\
\hline & 1 & 2 & 3 & $\begin{array}{l}\text { Tranquilizers: quaalude, seconal ("reds"), valium, } \\
\text { xanax ("bars"), librium, ativan, dalmane, halcion, } \\
\text { barbiturates, miltown, GHB, roofinol, "roofies" }\end{array}$ \\
\hline & 1 & 2 & 3 & $\begin{array}{l}\text { Miscellaneous: steroids, non-prescription sleep or diet } \\
\text { pills. cough medicine (without codeine). any others? }\end{array}$ \\
\hline
\end{tabular}

J2. Ask about the drug that caused the most problems, both past 12 months and lifetime

\begin{tabular}{|c|c|c|c|c|}
\hline \multirow{3}{*}{$\begin{array}{l}\text { Considering your use of (name/ class of selected drug): } \\
\text { a. During the times when you used the drug, did you end up } \\
\text { using more (name/ class of selected drug) than you planned } \\
\text { when started? }\end{array}$} & \multirow{2}{*}{\multicolumn{2}{|c|}{$\begin{array}{c}\begin{array}{c}\text { Drug } \\
\text { (write below): }\end{array} \\
\begin{array}{c}\text { In the past } 12 \\
\text { months: }\end{array}\end{array}$}} & \multirow{2}{*}{\multicolumn{2}{|c|}{$\begin{array}{c}\begin{array}{c}\text { Drug } \\
\text { (write below): }\end{array} \\
\text { Lifetime: }\end{array}$}} \\
\hline & & & & \\
\hline & $\mathrm{NO}$ & YES & NO & YES \\
\hline $\begin{array}{l}\text { b. Did you repeatedly want to reduce or control your (name/ } \\
\text { class of selected drug) use? } \\
\text { Did you try to cut down or control your (name/ class of } \\
\text { selected drug) use, but failed? } \\
\text { IF YES TO EITHER, CODE YES. }\end{array}$ & $\mathrm{NO}$ & YES & NO & YES \\
\hline $\begin{array}{l}\text { c. On the days that you used more (name/ class of selected } \\
\text { drug), did you spend substantial time obtaining it, using it, or } \\
\text { recovering from its effects? }\end{array}$ & NO & YES & NO & YES \\
\hline $\begin{array}{l}\text { d. Did you crave or have a strong desire or urge to use } \\
\text { (name/ class of selected drug)? }\end{array}$ & NO & YES & NO & YES \\
\hline
\end{tabular}


e. Have/did you missed work or school or often arrive late

NO

YES

NO

YES

(or do a bad job) because you were high or recovering from

the use? Did you stop meeting obligations at home (e.g., taking care of kids)?

f. If your (name/ class of selected drug) use caused problems with your family or other people, did you still keep on using it?

g. Did you use the drug more than once in any situation where you or others were physically at risk, for example, driving a car, riding a motorcycle, using machinery, boating, etc.?

h. Did you continue to use (name/ class of selected drug), even though it was clear that the it had caused or worsened psychological or physical problems?

i. Did you reduce or give up important work, social or recreational activities because of your (name/ class of selected drug)use?

j. Did you need to use (name/ class of selected drug) a lot more in order to get the same effect that you got when you first started using it, or did you get much less effect with continued use of the same amount?

J2K SUMMARY: IF YES TO J2K1 OR J2K2, CODE YES

NO YES NO YES

NO YES NO YES

NO YES NO YES

NO YES NO YES

k1. When you cut down on heavy or prolonged use of the drug did you have any of the following (see below for symptoms of (name/ class of selected drug)

withdrawal:

IF YES TO THE REQUIRED NUMBER OF WITHDRAWAL SYMPTOMS FOR EACH CLASS, CODE J2K1 AS YES.

Sedative, Hypnotic, Anxiolytic (2 or more)

1. Increased sweating or increased heart rate

2. Hand tremor or "the shakes"

3. Trouble sleeping

4. Nausea or vomiting

5. Hearing or seeing things other people could not see or hear or having sensations in your skin for no apparent reason

6. Agitation

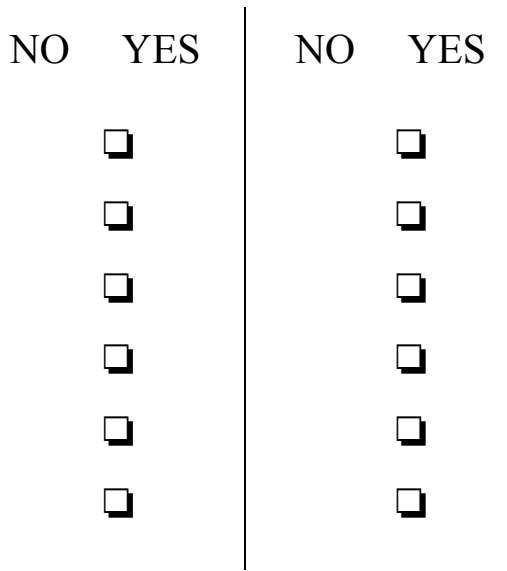


7. Anxiety

8. Seizures

\section{Opiates ( 3 or more)}

1. Feeling depressed

2. Nausea or vomiting

3. Muscle aches

4. Runny nose or teary eyes

5. Dilated pupils, goose bumps or hair standing on end or sweating

6. Diarrhea

7. Yawning

8. Hot Flashes

9. Trouble sleeping

\section{Stimulants (2 or more)}

1. Fatigue

2. Vivid or unpleasant dreams

3. Difficulty sleeping or sleeping too much

4. Increased appetite

5. Feeling or looking physically or mentally slowed down

\section{Cannabis (3 or more)}

1. Irritability

2. Nervousness or anxiety

3. Trouble sleeping

4. Appetite or weight loss

5. Restlessness

6. Feeling depressed

7. Significant discomfort from one of the following: "stomach pain", tremors or "shakes", sweating, hot flashes, chills, headaches

k2. Did you use (name/ class of selected drug) to reduce or avoid withdrawal symptoms?

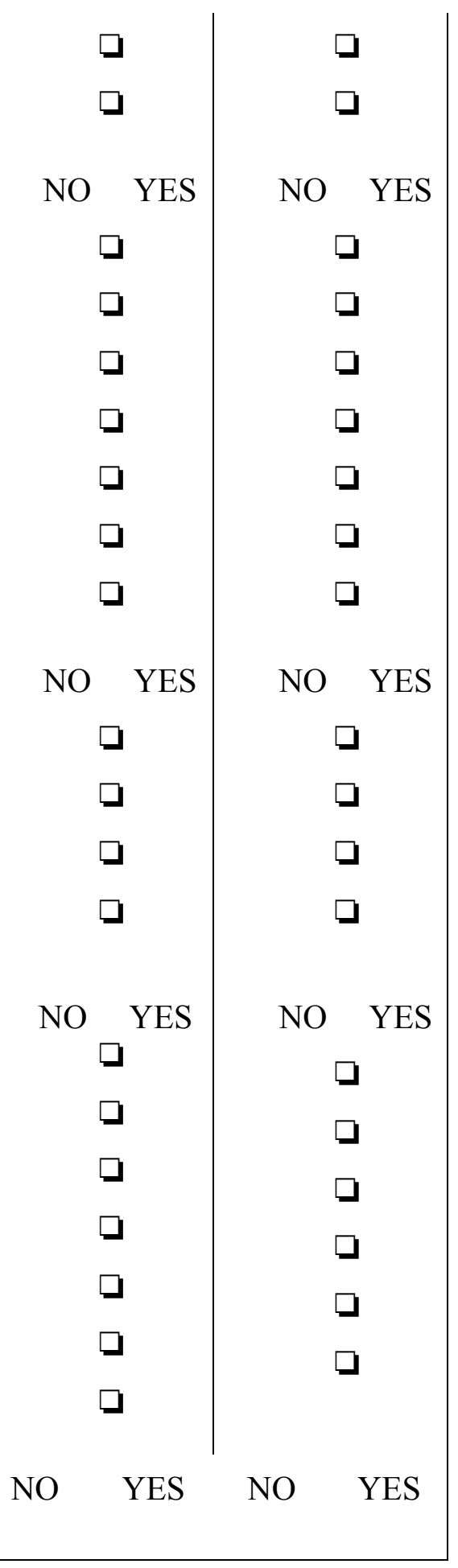

\section{PAST 12 MONTHS:}

Are 2 or more $\mathrm{J} 2$ answers from $\mathrm{J} 2 \mathrm{a}-\mathrm{k}$ summary coded yes? (J2k1 \& J2k2 together count as one)
SUD (PAST 12 MONTHS)

NO

YES 
SPECIFIERS FOR SUBSTANCE USE DISORDER:

Mild $=2-3$ of the $\mathrm{J} 2$ symptoms

Moderate $=4--5$ of the $\mathrm{J} 2$ symptoms

Severe $=6$ or more of the $\mathrm{J} 2$ symptoms

In early remission $=$ criteria not met for between 3

\& 12 months

In sustained remission $=$ criteria not met for 12

months or more (both with the exception of

criterion d. - (craving) above).

In a controlled environment $=$ where substance/

drug access is restricted
Specify if:

Mild

Moderate

Severe

In early remission

$\square$ In sustained

remission

$\square$ In a controlled environment

\section{LIFETIME}

Are 2 or more $\mathrm{J} 2$ answers from $\mathrm{J} 2 \mathrm{a}-\mathrm{k}$ summary coded yes? (J2k1 \& J2k2 together count as one)

SPECIFIERS FOR SUBSTANCE USE DISORDER:

Mild $=2-3$ of the $\mathrm{J} 2$ symptoms

Moderate $=4--5$ of the 2 symptoms

Severe $=6$ or more of the $\mathrm{J} 2$ symptoms

In early remission $=$ criteria not met for between 3

\& 12 months

In sustained remission $=$ criteria not met for 12

months or more (both with the exception of

criterion d. - (craving) above).

In a controlled environment $=$ where substance/ drug access is restricted

\section{SUD (LIFETIME)}

NO

YES

Specify if:

Mild

$\square$ Moderate

$\square$ Severe

$\square$ In early remission

$\square$ In sustained remission

In a controlled environment 


\section{APPENDIX K. \\ Mood and Anxiety Symptom Questionnaire (MASQ)}

Please rate how much you were experiencing each of the following in the last 2 weeks:

Not at all

a bit

Moderately

Quite

1. Felt really good about myself

2. Felt optimistic

3. Seemed to move quickly and easily

4. Felt cheerful

5. Felt really "up" and lively

6. Felt like my heart was racing or pounding

7. Felt like nothing was very enjoyable

8. Was afraid I was going to die

9. Was trembling or shaking

10. Felt unattractive

11. Felt faint

12. Had to urinate frequently

13. Felt like I had a lot of energy

14. Felt like there wasn't anything interesting or fun to do

15. Felt like I had a lot of interesting things to do

16. Felt dizzy or lightheaded

17. Was proud of myself

18. Felt like I had accomplished a lot

19. Was short of breath

20. Felt really slowed down

21. Felt like I had a lot to look forward to

22. Felt like it took extra effort to get started

23. Felt numbness or tingling in my body

24. Hands were cold or sweaty

25. Looked forward to things with enjoyment

26. Felt really happy

27. Hands were shaky

28. Startled easily

29. Had hot or cold spells

30. Had trouble swallowing

31. Muscles twitched or trembled

32. Thought about death or suicide

33. Had a very dry mouth 
34. Had pain in my chest

35. Felt really bored

36. Felt withdrawn from people

37. Felt like I was choking

38. Felt hopeful about the future

39. Felt like I was having a lot of fun 


\section{APPENDIX L. \\ Penn State Worry Questionnaire (PSWQ)}

\begin{tabular}{|l|l|l|l|l|l|l|l|}
\hline \multicolumn{2}{|l}{} & \multicolumn{2}{|c|}{$\begin{array}{l}\text { Not at all } \\
\text { typical of } \\
\text { me }\end{array}$} & \multicolumn{2}{|c|}{$\begin{array}{c}\text { Very } \\
\text { typical } \\
\text { of me }\end{array}$} \\
\hline 1 & $\begin{array}{l}\text { If I do not have enough time to do everything, I do not } \\
\text { worry about it. }\end{array}$ & 1 & 2 & 3 & 4 & 5 \\
\hline 2 & My worries overwhelm me. & 1 & 2 & 3 & 4 & 5 \\
\hline 3 & I do not tend to worry about things. & 1 & 2 & 3 & 4 & 5 \\
\hline 4 & Many situations make me worry. & 1 & 2 & 3 & 4 & 5 \\
\hline 5 & $\begin{array}{l}\text { I know I should not worry about things, but I just } \\
\text { cannot help it. }\end{array}$ & 1 & 2 & 3 & 4 & 5 \\
\hline 6 & When I am under pressure, I worry a lot. & 1 & 2 & 3 & 4 & 5 \\
\hline 7 & I am always worrying about something. & 1 & 2 & 3 & 4 & 5 \\
\hline 8 & I find it easy to dismiss worrisome thoughts. & 1 & 2 & 3 & 4 & 5 \\
\hline 9 & $\begin{array}{l}\text { As soon as I finish one task, I start to worry about } \\
\text { everything else I have to do. }\end{array}$ & 1 & 2 & 3 & 4 & 5 \\
\hline 10 & I never worry about anything. & 1 & 2 & 3 & 4 & 5 \\
\hline 11 & $\begin{array}{l}\text { When there is nothing more I can do about a concern, I } \\
\text { do not worry about it anymore. }\end{array}$ & 1 & 2 & 3 & 4 & 5 \\
\hline 12 & I have been a worrier all my life. & 1 & 2 & 3 & 4 & 5 \\
\hline 13 & I notice that I have been worrying about things. & 1 & 2 & 3 & 4 & 5 \\
\hline 14 & Once I start worrying, I cannot stop. & 1 & 2 & 3 & 4 & 5 \\
\hline 15 & I worry all the time. & 2 & 3 & 4 & 5 \\
\hline 16 & I worry about projects until they are all done. & 3 & 4 & 5 \\
\hline & & & & & & & \\
\hline
\end{tabular}




\section{APPENDIX M. \\ Post-NPU Task Questionnaire}

Please rate your overall lab experience today

1. How attentive were you during the experiment?

$\begin{array}{ccccc}0 & 1 & 2 & 3 & 4 \\ \text { Not Attentive } & & & & \text { Very Attentive }\end{array}$

2. How sleepy were you during the experiment?

$\begin{array}{ccccc}0 & 1 & 2 & 3 & 4 \\ \text { Not sleepy at all } & & & & \text { Very Sleepy }\end{array}$

3. How intense was the shock?

$\begin{array}{ccccc}1 & 1 & 3 & 4 \\ \text { Very Mild } & & & & \text { High }\end{array}$

4. How anxiety/fear provoking was the shock?

$\begin{array}{ccccc}\text { L } & 1 & 2 & 3 & 4 \\ \text { Very Mild } & & & & \text { High }\end{array}$

5. How painful was the shock?

$\begin{array}{ccccc}0 & 1 & 2 & 3 & 4 \\ \text { Not at all } & & & & \text { Very Painful }\end{array}$




\section{NO SHOCKS}

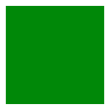

6. How anxious/fearful were you during the No Shock block while you were seeing the green square?

\begin{tabular}{|c|c|c|c|c|}
\hline 0 & 1 & 2 & 3 & 4 \\
\hline Not Anxious & & & & \\
\hline
\end{tabular}

\section{PREDICTABLE SHOCKS}

7. How anxious/fearful were you during the Predictable Shock block while you were seeing the red square?

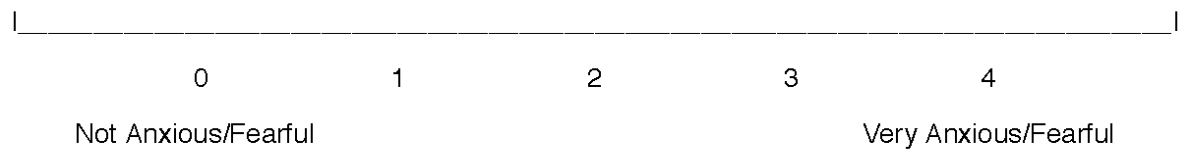

\section{UNPREDICTABLE SHOCKS}

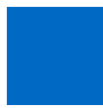

8. How anxious/fearful were you during the Unpredictable Shock block while you were seeing the blue square?

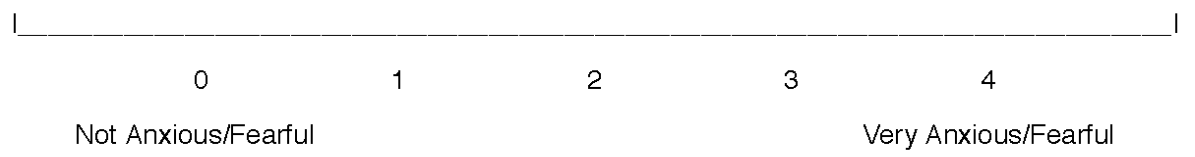




\section{APPENDIX N. \\ Positive and Negative Affect Schedule (PANAS)}

Session Number: $1 \quad 2 \quad$ PANAS Number:

Instructions: This scale consists of a number of words that describe different feelings and emotions. Read each item and then list the number from the scale below next to each word. Indicate to what extent you feel this way right now, that is, at the present moment.

1

2

3

4

5

Very slightly or

A Little

Moderately

Quite a Bit

Extremely

Not at All

1. Interested

2. Distressed

3. Excited

4. Upset

5. Strong

6. Guilty

7. Scared

8. Hostile

9. Enthusiastic

10. Proud
11. Irritable

12. Alert

13. Ashamed

14. Inspired

15. Nervous

16. Determined

17. Attentive

18. Jittery

19. Active

20. Afraid 


\section{APPENDIX 0. Supplementary Materials}

Self-Reported Affect Throughout the Session. Participants' moods were assessed using the Positive and Negative Affect (PANAS) (Watson, Clark, \& Tellegen, 1988; see Appendix N) in order to assess changes in affect across the study session. Specifically, the PANAS was administered 1) immediately upon arrival (prior to consenting), 2) following the clinical interviews and after EEG capping, and 3) following the NPU-ANT task. The PANAS consists of two 10-item scales measuring positive affect (PA; e.g., "enthusiastic", "alert") and negative affect (NA; e.g., "scared", "upset"). Participants rated their current mood on a 5-point scale (ranging from $1=$ "very slightly or not at all" to 5 = "extremely"). The PANAS has been found to be sensitive to fluctuations in mood (Watson, Clark, \& Tellegan, 1988) and was used to assess changes in mood (particularly increases in negative affect) throughout the session and from before to after the NPU-ANT Task.

We computed a series of repeated measures GLMs to examine the effect of time (3 time points: start of session, after EEG capping, and after the NPU-ANT task) on PANAS ratings of positive and negative affect across the session, relying on a-priori planned contrasts (ordered: start of session, after NPU-ANT task, after capping). Linear effects indexed increased or decreased affect between the start of the session and prior to the start of the NPU-ANT task; however, we were mainly interested in the quadratic effects, which indexed increased or decreased affect post-NPU-ANT task compared to the two baselines, as it were (i.e. start of session and pre-NPU-ANT task). See Figure S1. 


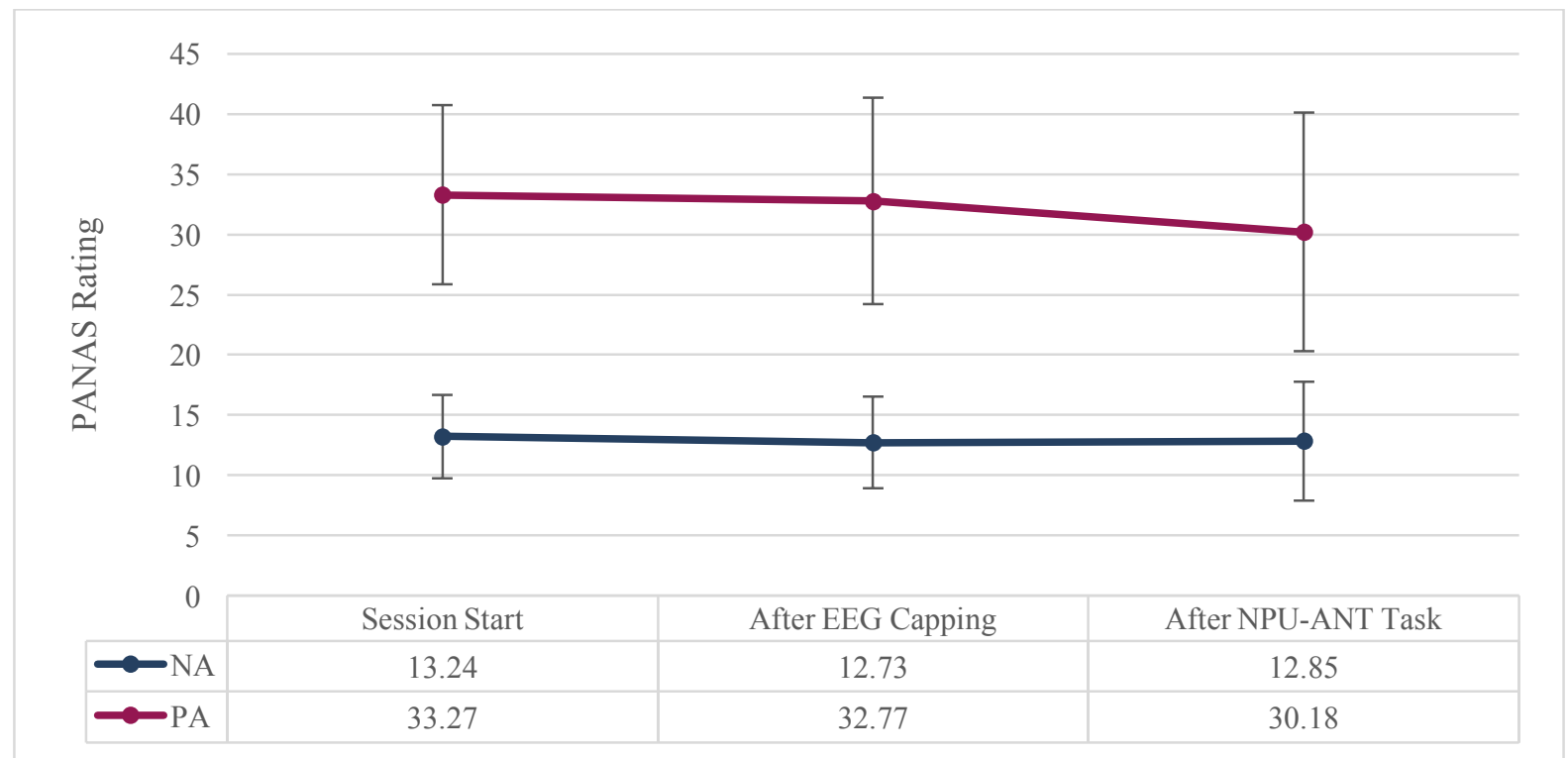

Figure S1. Average PANAS Affect Scores Across Session.

For negative affect (NA) ratings, analyses revealed a small but significant linear effect of time on NA, $F(1,142)=5.31, p>.02, \eta_{\mathrm{p}}{ }^{2}=.04$, indicating that NA went down from start of session to after the interview and EEG capping. There was not a significant quadratic effect of time on NA, suggesting NA following the NPU-ANT task was not markedly different compared to the two baselines. However, examining the pattern of effects of time on specific emotions, we found that after the NPU-ANT task (vs. other time points) participants reported that they were significantly less scared and nervous and significantly more jittery, distressed, and irritable.

For positive affect $(\mathrm{PA})$, a significant quadratic effect of time, $F(1,142)=36.35, p<.00$, $\eta_{\mathrm{p}}{ }^{2}=.20$, indicated reduced PA following the NPU-ANT task compared to the two baseline time points (i.e. start of session, after interviews/ EEG capping). Specifically, participants reported significantly lower levels of self-reported interest, excitement, strength, enthusiasm, pride, alertness, inspiration, attention, and activeness (and increased levels of determination) following the NPU-ANT task compared to prior points in the session. Finally, we did not find a linear effect of time on PA, suggesting PA did not significantly differ between the start of the session to following the clinical interviews/ EEG capping. 
Together these results indicate that following the task, participants responses indicated that they felt less anticipatory worry (less scared and nervous) but also more physical discomfort (more jittery, distressed, irritable). They also had overall significant decreases in PA, which is to be expected given that the task was designed to be both emotionally and cognitively taxing.

Alternative P3 Site Analyses. For Aim 1, we conducted a series of mixed-model repeated measures Threat x Congruence GLMs on frontocentral P3 and central P3. Again, we focused on polynomial contrasts of threat (un)predictability ( $P$ vs. $U$ ) and overall threat (vs. no threat; $P / U$ vs. N). Results of these analyses are presented in Table S1 and Table 2 in main text provides a summary of condition means.

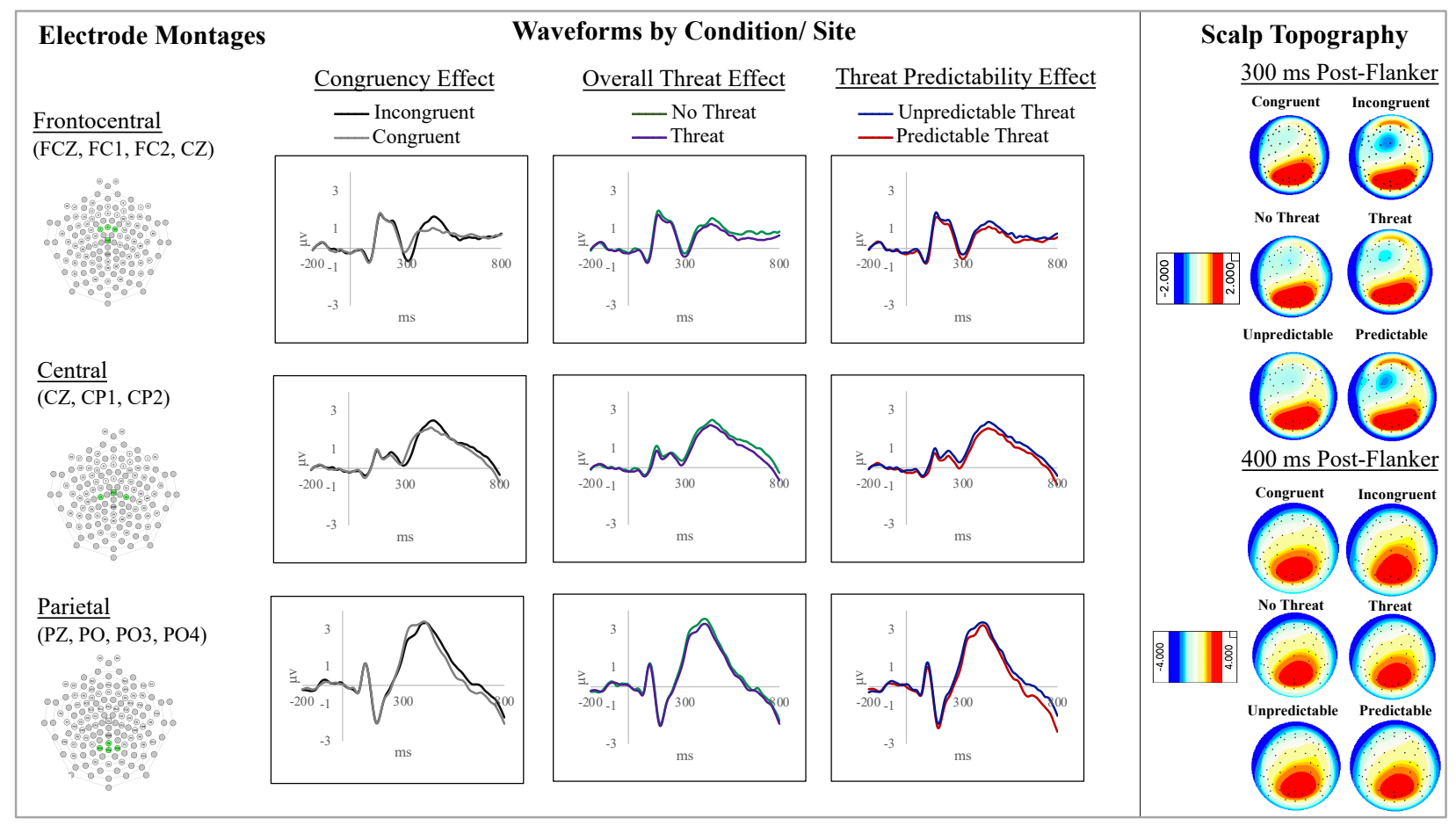

Figure S2. ERP Waveforms and Topographies by Condition and Site.

Consistent across all P3 sites was the significant overall effect of threat (P/U vs. N;

frontocentral: $F(1,113)=4.43, p=.04, \eta 2=0.04$; central: $F(1,113)=7.48, p<.01, \eta 2=0.06)$

such that the P3 was smaller (less positive) during threat versus no threat contexts. In addition to the effect of overall threat, effect of threat (un)predictability (P vs. U) was also present at the 
central site $(F(1,113)=10.23, p<.01, \eta 2=0.08)$, such that $\mathrm{P} 3$ amplitude was significantly larger (more positive) during unpredictable (vs. predictable) threat contexts. Of note, the frontocentral $\mathrm{P} 3$ also showed a similar pattern of threat (un)predictability, though it was smaller, $F(1,113)=$ $2.98, p=.09 ; \eta_{\mathrm{p}}{ }^{2}=.03$. Consistent with the results of our site analyses, these results suggest that the effects of threat on the $\mathrm{P} 3$ was similar to across all P3 sites.

Different from results of parietal P3 analyses, we observed a significant congruence effect at both frontocentral and central $\mathrm{P} 3$ sites (i.e., larger/more positive $\mathrm{P} 3$ for incongruent vs. congruent flanker trials; frontocentral: $F(1,113)=27.23, p<.001, \eta 2=0.19$; central: $F(1,113)=$ $7.89, p<.01, \eta 2=0.07)$. Also interesting, the central $\mathrm{P} 3$ had a small, marginally significant interaction of overall threat $(\mathrm{P} / \mathrm{U}$ vs. $\mathrm{N})$ by flanker congruence, $F(1,113)=3.01, p=.09, \eta_{\mathrm{p}}{ }^{2}=$ .03 , such that flanker congruence differentiation was actually larger during threat (vs. no threat) contexts (threat incongruent $M=3.17$; threat congruent $M=2.82$; no threat incongruent $M=$ 3.26; no threat congruent $M=3.14$ ). This result may suggest that threat facilitates congruence differentiation, although this effect was small in size and its meaningfulness is difficult to determine.

Table S1. GLM Effects Within Site Frontocentral and Central P3.

\begin{tabular}{lllll}
\hline & $\boldsymbol{F}$ & $\mathbf{d f}$ & $\boldsymbol{p}$ & $\boldsymbol{\eta}_{\mathbf{p}}{ }^{2}$ \\
\hline Frontocentral Site - P3 & & & & \\
\hline Threat Predictability & 2.98 & $(1,113)$ & $.09^{\wedge}$ & .03 \\
Overall Threat & 4.43 & $(1,113)$ & $.04^{*}$ & .04 \\
Congruence & 27.23 & $(1,113)$ & $.00^{* * *}$ & .19 \\
Threat Predictability x Congruence & 0.46 & $(1,113)$ & .50 & .00 \\
Overall Threat x Congruence & 0.71 & $(1,113)$ & .40 & .01 \\
\hline Central Site $-\mathbf{P 3}$ & & & & \\
\hline Threat Predictability & 10.23 & $1,113)$ & $.00^{* *}$ & .08 \\
Overall Threat & 7.48 & $1,113)$ & $.01^{* *}$ & .06 \\
Congruence & 7.89 & $1,113)$ & $.01^{* *}$ & .07 \\
Threat Predictability Threat x Congruence & 0.00 & $1,113)$ & .98 & .00 \\
Overall Threat x Congruence & 3.01 & $1,113)$ & $.09^{\wedge}$ & .03 \\
\hline
\end{tabular}

Note $-{ }^{*} p<.05,{ }^{* *} p<.01,{ }^{* * *} p<.001, \wedge$ marginal effect $(p<.10)$. 
For Aim 2, we added each measure of disinhibited behavior was added a continuous between-subjects factor in separate Threat x Congruence GLMs for both frontocentral P3 and central P3.

Disinhibition Factor. In contrast to parietal P3 analyses which revealed both a two-way Disinhibition x Overall Threat effect as well as a Disinhibition x Overall Threat x Congruence effect, analyses of the frontocentral P3 and central P3 revealed no effects involving the disinhibition factor (see Table S2).

Table S2. GLM Effects of Frontocentral and Central P3 as a function of Disinhibition.

\begin{tabular}{lllll} 
& $\boldsymbol{F}$ & $\mathbf{d f}$ & $\boldsymbol{p}$ & $\boldsymbol{\eta}_{\mathbf{p}}{ }^{2}$ \\
\hline Frontocentral Site - P3 & & & & \\
\hline Threat Predictability & 2.54 & $(1,109)$ & .11 & .02 \\
Overall Threat & 3.68 & $(1,109)$ & $.06^{\wedge}$ & .03 \\
Congruence & 24.65 & $(1,109)$ & $.00^{* * *}$ & .18 \\
Disinhibition (Between Subjects) & .003 & $(1,109)$ & .96 & .00 \\
Threat Predictability x Disinhibition & .08 & $(1,109)$ & .77 & .00 \\
Overall Threat x Disinhibition & .57 & $(1,109)$ & .45 & .01 \\
Congruence x Disinhibition & .61 & $(1,109)$ & .44 & .01 \\
Threat Predictability x Congruence & .39 & $(1,109)$ & .53 & .00 \\
Overall Threat x Congruence & .62 & $(1,109)$ & .43 & .01 \\
Threat Predictability x Congruence x Disinhibition & .15 & $(1,109)$ & .70 & .00 \\
Overall Threat x Congruence x Disinhibition & 1.97 & $(1,109)$ & .16 & .02 \\
\hline Central Site - P3 & & & & \\
\hline Threat Predictability & 9.58 & $(1,109)$ & $.00 * *$ & .08 \\
Overall Threat & 6.35 & $(1,109)$ & $.01 *$ & .06 \\
Congruence & 6.73 & $(1,109)$ & $.01 *$ & .06 \\
Disinhibition (Between Subjects) & .14 & $(1,109)$ & .71 & .00 \\
Threat Predictability x Disinhibition & .40 & $(1,109)$ & .55 & .00 \\
Overall Threat x Disinhibition & .02 & $(1,109)$ & .90 & .00 \\
Congruence x Disinhibition & .26 & $(1,109)$ & .61 & .00 \\
Threat Predictability x Congruence & .00 & $(1,109)$ & .99 & .00 \\
Overall Threat x Congruence & 2.24 & $(1,109)$ & .14 & .02 \\
Threat Predictability x Congruence x Disinhibition & .44 & $(1,109)$ & .51 & .00 \\
Overall Threat x Congruence x Disinhibition & 1.76 & $(1,109)$ & .19 & .02 \\
\hline
\end{tabular}

Note. ${ }^{*} p<.05,{ }^{* *} p<.01,{ }^{* * *} p<.001,{ }^{\wedge}$ marginal effect $(p>.05)$.

Negative Urgency. Whereas analyses of the parietal P3 revealed no effects involving negative urgency, analyses of the frontocentral and central P3 revealed significant two-way 
Negative Urgency x Overall Threat (P/U vs. N) interactions at both sites (frontocentral:

$F(1,109)=7.24, p=.01, \eta_{\mathrm{p}}{ }^{2}=.06$; central: $\left.F(1,109)=4.0, p=.04, \eta_{\mathrm{p}}{ }^{2}=.04\right)$. See Table S3. Like the observed effect of negative urgency on N2 amplitude, we found that higher scores on negative urgency were associated with reduced (smaller, less positive) P3 processing of flankers during conditions of threat (frontocentral: $r=-.05, p=.61$; central: $r=-.03, p=.80$ ) and enhanced (larger, more positive) $\mathrm{P} 3$ processing of flankers during conditions of no threat (frontocentral: $r=$ $.07, p=.46$, central: $r=.05, p=.57$ ), resulting in a slightly larger threat effect with higher scores on negative urgency. See Figure S3 for a demonstration of this effect at the frontocentral site.

Table S3. GLM Effects of Frontocentral and Central P3 as a function of NU.

\begin{tabular}{lllll}
\hline & $\boldsymbol{F}$ & $\mathbf{d f}$ & $\boldsymbol{p}$ & $\boldsymbol{\eta}_{\mathbf{p}}{ }^{2}$ \\
\hline Frontocentral Site - P3 & & & & \\
\hline Threat Predictability & .15 & $(1,109)$ & .70 & .00 \\
Overall Threat & 3.47 & $(1,109)$ & $.06^{\wedge}$ & .03 \\
Congruence & 1.15 & $(1,109)$ & .29 & .01 \\
NU (Between Subjects) & .10 & $(1,109)$ & .75 & .00 \\
Threat Predictability x NU & .03 & $(1,109)$ & .87 & .00 \\
Overall Threat x NU & 7.24 & $(1,109)$ & $.01^{* *}$ & .06 \\
Congruence x NU & .38 & $(1,109)$ & .54 & .00 \\
Threat Predictability x Congruence & .06 & $(1,109)$ & .82 & .00 \\
Overall Threat x Congruence & .68 & $(1,109)$ & .41 & .01 \\
Threat Predictability x Congruence x NU & .00 & $(1,109)$ & .97 & .00 \\
Overall Threat x Congruence x NU & 1.37 & $(1,109)$ & .24 & .01 \\
\hline Central Site - P3 & & & & \\
\hline Threat Predictability & .29 & $(1,109)$ & .59 & .00 \\
Overall Threat & 1.23 & $(1,109)$ & .27 & .01 \\
Congruence & .20 & $(1,109)$ & .65 & .00 \\
NU (Between Subjects) & .00 & $(1,109)$ & .96 & .00 \\
Threat Predictability x NU & .28 & $(1,109)$ & .60 & .00 \\
Overall Threat x NU & 4.30 & $(1,109)$ & $.04 *$ & .04 \\
Congruence x NU & .19 & $(1,109)$ & .66 & .00 \\
Threat Predictability x Congruence & 1.66 & $(1,109)$ & .20 & .02 \\
Overall Threat x Congruence & .45 & $(1,109)$ & .50 & .00 \\
Threat Predictability x Congruence x NU & 1.91 & $(1,109)$ & .17 & .02 \\
Overall Threat x Congruence x NU & 1.60 & $(1,109)$ & .21 & .01 \\
\hline
\end{tabular}

Note. ${ }^{*} p<.05,{ }^{* *} p<.01,{ }^{* * *} p<.001,{ }^{\wedge}$ marginal effect $(p>.05)$. 


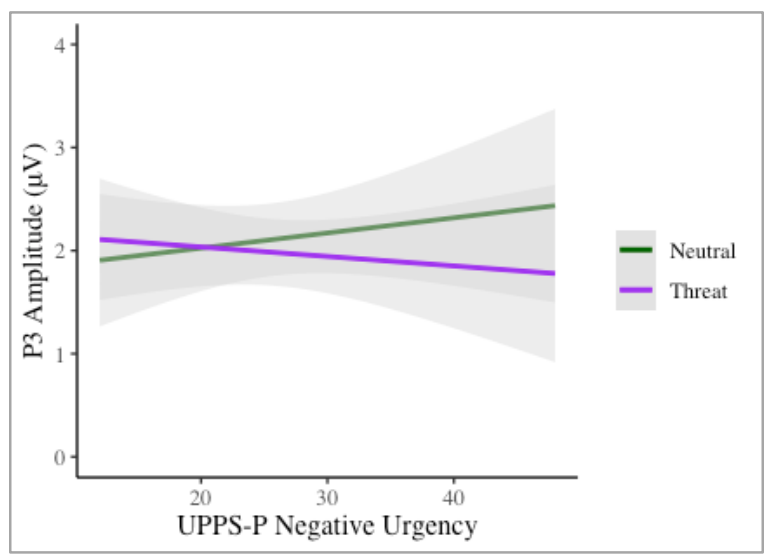

Figure S3. Negative Urgency x Overall Threat Interaction at Frontocentral P3.

Aggression. Analyses revealed no significant effects involving the aggressive behavior for the frontocentral P3 or central P3 (see Table S4). However at the central P3 site, there was a small, marginally significant two-way Aggression x Threat Predictability (P vs. U) interaction, $F(1,112)=3.21, p=.08, \eta_{\mathrm{p}}{ }^{2}=.03$. Follow-up correlations revealed that increasing levels of aggressive behavior were associated with decreases in central P3 amplitude, that were less pronounced during unpredictable $(r=-.05, p=.56)$ compared to predictable $(r=-.13, p=.17)$ threat. See Figure S4 for an illustration of this effect. Of note, these results are different from the parietal P3 results which revealed both a two-way Aggression x Overall Threat interaction and a marginally significant three-way Aggression x Overall Threat (P/U vs. N) x Congruence interaction.

Table S4. GLM Effects of Frontocentral and Central P3 as a function of Aggressive Behavior.

\begin{tabular}{lllll}
\hline Frontocentral Site - P3 & $\boldsymbol{F}$ & $\mathbf{d f}$ & $\boldsymbol{p}$ & $\boldsymbol{\eta}_{\mathbf{p}}{ }^{2}$ \\
\hline Threat Predictability & & & & \\
Overall Threat & .13 & $(1,112)$ & .72 & .01 \\
Congruence & .80 & $(1,112)$ & .38 & .01 \\
Aggression (Between Subjects) & 7.36 & $(1,112)$ & $.01^{* *}$ & .06 \\
Threat Predictability x Aggression & .66 & $(1,112)$ & .42 & .01 \\
Overall Threat x Aggression & 1.71 & $(1,112)$ & .19 & .02 \\
Congruence x Aggression & .01 & $(1,112)$ & .93 & .00 \\
Threat Predictability x Congruence & .12 & $(1,112)$ & .73 & .00 \\
Overall Threat x Congruence & .15 & $(1,112)$ & .70 & .00 \\
Threat Predictability x Congruence x Aggression & 1.01 & $(1,112)$ & .32 & .01 \\
& .01 & $(1,112)$ & .93 & .00
\end{tabular}


Table S4. (Continued)

\begin{tabular}{lllll} 
Overall Threat x Congruence x Aggression & 2.49 & $(1,112)$ & .12 & .02 \\
\hline Central Site - P3 & & & & \\
\hline Threat Predictability & .01 & $(1,112)$ & .92 & .00 \\
Overall Threat & 3.47 & $(1,112)$ & $.07^{\wedge}$ & .03 \\
Congruence & 4.41 & $(1,112)$ & $.04^{*}$ & .04 \\
Aggression (Between Subjects) & 1.38 & $(1,112)$ & .24 & .01 \\
Threat Predictability x Aggression & 3.21 & $(1,112)$ & $.08^{\wedge}$ & .03 \\
Overall Threat x Aggression & .46 & $(1,112)$ & .50 & .00 \\
Congruence x Aggression & .82 & $(1,112)$ & .37 & .01 \\
Threat Predictability x Congruence & .24 & $(1,112)$ & .64 & .00 \\
Overall Threat x Congruence & .18 & $(1,112)$ & .67 & .00 \\
Threat Predictability x Congruence x Aggression & .30 & $(1,112)$ & .59 & .00 \\
Overall Threat x Congruence x Aggression & 1.92 & $(1,112)$ & .17 & .02 \\
\hline
\end{tabular}

Note. ${ }^{*} p<.05,{ }^{* *} p<.01,{ }^{* * *} p<.001,{ }^{\wedge}$ marginal effect $(p>.05)$.

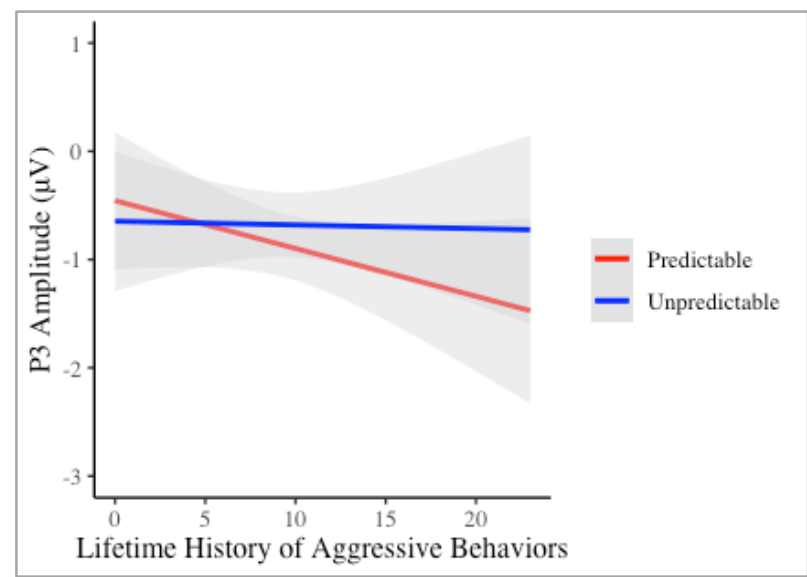

Figure S4. Aggression x Threat Predictability Interaction at Central P3.

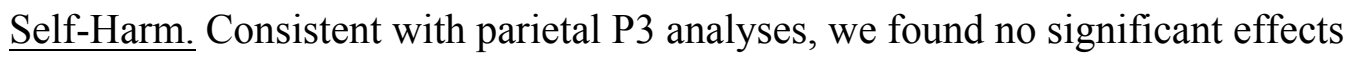

involving self-harm behavior at frontocentral or central P3 sites. See Table S5.

Table S5. GLM Effects of Frontocentral and Central P3 as a function of Self-Harm.

\begin{tabular}{lllll}
\hline & $\boldsymbol{F}$ & $\mathbf{d f}$ & $\boldsymbol{p}$ & $\boldsymbol{\eta}_{\mathbf{p}}{ }^{2}$ \\
\hline Frontocentral Site - P3 & & & & \\
\hline Threat Predictability & 3.00 & $(1,112)$ & $.09^{\wedge}$ & .03 \\
Overall Threat & 1.18 & $(1,112)$ & .28 & .01 \\
Congruence & 14.20 & $(1,112)$ & $.00^{* * *}$ & .11 \\
Self-Harm (Between Subjects) & 1.36 & $(1,112)$ & .25 & .01 \\
Threat Predictability x Self-Harm & .27 & $(1,112)$ & .60 & .00 \\
Overall Threat x Self-Harm & 1.63 & $(1,112)$ & .20 & .01 \\
Congruence x Self-Harm & 1.35 & $(1,112)$ & .25 & .01 \\
Threat Predictability x Congruence & 1.94 & $(1,112)$ & .17 & .02
\end{tabular}


Table S5. (Continued)

\begin{tabular}{lllll} 
Overall Threat x Congruence & .38 & $(1,112)$ & .54 & .00 \\
Threat Predictability x Congruence x Self-Harm & 2.30 & $(1,112)$ & .13 & .02 \\
Overall Threat x Congruence x Self-Harm & .03 & $(1,112)$ & .86 & .00 \\
\hline Central Site - P3 & & & & \\
\hline Threat Predictability & 12.94 & $(1,112)$ & $.00^{* * *}$ & .10 \\
Overall Threat & 6.5 & $(1,112)$ & $.01^{*}$ & .06 \\
Congruence & 2.45 & $(1,112)$ & .12 & .02 \\
Self-Harm (Between Subjects) & .19 & $(1,112)$ & .67 & .00 \\
Threat Predictability x Self-Harm & 2.69 & $(1,112)$ & .10 & .02 \\
Overall Threat x Self-Harm & .23 & $(1,112)$ & .63 & .00 \\
Congruence x Self-Harm & 2.26 & $(1,112)$ & .14 & .02 \\
Threat Predictability x Congruence & .12 & $(1,112)$ & .73 & .00 \\
Overall Threat x Congruence & 1.81 & $(1,112)$ & .18 & .02 \\
Threat Predictability x Congruence x Self-Harm & .47 & $(1,112)$ & .50 & .00 \\
Overall Threat x Congruence x Self-Harm & .04 & $(1,112)$ & .84 & .00 \\
\hline
\end{tabular}

Note. ${ }^{*} p<.05,{ }^{* *} p<.01,{ }^{* * *} p<.001,{ }^{\wedge}$ marginal effect $(p>.05)$. Self-harm was log transformed.

AUD Symptoms. Consistent with parietal P3 analyses, we found no significant effects

involving lifetime Alcohol Use Disorder (AUD) symptoms at frontocentral or central P3 sites.

See Table S6.

Table S6. GLM Effects of Frontocentral and Central P3 as a function of AUD Symptoms.

\begin{tabular}{lllll}
\hline & $\boldsymbol{F}$ & $\mathbf{d f}$ & $\boldsymbol{p}$ & $\mathbf{\eta}_{\mathbf{p}}{ }^{2}$ \\
\hline Frontocentral Site - P3 & & & & \\
\hline Threat Predictability & 4.86 & $(1,112)$ & $.03^{*}$ & .04 \\
Overall Threat & 5.63 & $(1,112)$ & $.02^{*}$ & .05 \\
Congruence & 10.06 & $(1,112)$ & $.00^{* *}$ & .08 \\
AUD (Between Subjects) & .28 & $(1,112)$ & .60 & .00 \\
Threat Predictability x AUD & 1.94 & $(1,112)$ & .17 & .02 \\
Overall Threat x AUD & 1.60 & $(1,112)$ & .21 & .01 \\
Congruence x AUD & .34 & $(1,112)$ & .56 & .00 \\
Threat Predictability x Congruence & .03 & $(1,112)$ & .87 & .00 \\
Overall Threat x Congruence & .12 & $(1,112)$ & .73 & .00 \\
Threat Predictability x Congruence x AUD & .77 & $(1,112)$ & .38 & .01 \\
Overall Threat x Congruence x AUD & 1.65 & $(1,112)$ & .20 & .01 \\
\hline Central Site - P3 & & & & \\
\hline Threat Predictability & 6.55 & $(1,112)$ & $.01 *$ & .06 \\
Overall Threat & 5.26 & $(1,112)$ & $.02 *$ & .05 \\
Congruence & 2.47 & $(1,112)$ & .12 & .02 \\
AUD (Between Subjects) & 1.10 & $(1,112)$ & .30 & .01 \\
Threat Predictability x AUD & .24 & $(1,112)$ & .62 & .00 \\
Overall Threat x AUD & .32 & $(1,112)$ & .57 & .00 \\
Congruence x AUD & .25 & $(1,112)$ & .62 & .00
\end{tabular}


Threat Predictability x Congruence

Overall Threat $x$ Congruence

Threat Predictability x Congruence x AUD

Overall Threat $\mathrm{x}$ Congruence $\mathrm{x}$ AUD

Note. ${ }^{*} p<.05,{ }^{* *} p<.01,{ }^{* * *} p<.001, \wedge$ marginal effect $(p>.05)$.

$\begin{array}{llll}1.41 \quad(1,112) & .24 & .01\end{array}$

SUD Symptoms. Unlike parietal P3 analyses which revealed a significant three-way SUD

$x$ Overall Threat $(\mathrm{P} / \mathrm{U}$ vs. $\mathrm{N}) \mathrm{x}$ Congruence interaction, analyses of the frontocentral and central

P3 revealed no effects involving lifetime symptoms of SUD symptoms.

Table S7. GLM Effects of Frontocentral and Central P3 as a function of SUD Symptoms.

\begin{tabular}{lllll} 
& $\boldsymbol{F}$ & $\mathbf{d f}$ & $\boldsymbol{p}$ & $\boldsymbol{\eta}_{\mathbf{p}}{ }^{2}$ \\
\hline Frontocentral Site - P3 & & & & \\
\hline Threat Predictability & 1.76 & $(1,112)$ & .19 & .02 \\
Overall Threat & 1.56 & $(1,112)$ & .21 & .01 \\
Congruence & 8.44 & $(1,112)$ & $.00^{* *}$ & .07 \\
SUD (Between Subjects) & .67 & $(1,112)$ & .41 & .01 \\
Threat Predictability x SUD & .01 & $(1,112)$ & .94 & .00 \\
Overall Threat x SUD & .22 & $(1,112)$ & .64 & .00 \\
Congruence x SUD & 2.25 & $(1,112)$ & .14 & .02 \\
Threat Predictability x Congruence & .84 & $(1,112)$ & .36 & .01 \\
Overall Threat x Congruence & .00 & $(1,112)$ & .95 & .00 \\
Threat Predictability x Congruence x SUD & .39 & $(1,112)$ & .54 & .00 \\
Overall Threat x Congruence x SUD & .70 & $(1,112)$ & .40 & .01 \\
\hline Central Site - P3 & & & & \\
\hline Threat Predictability & 5.57 & $(1,112)$ & $.02 *$ & .05 \\
Overall Threat & 4.29 & $(1,112)$ & $.04 *$ & .04 \\
Congruence & 1.62 & $(1,112)$ & .21 & .01 \\
SUD (Between Subjects) & .39 & $(1,112)$ & .53 & .00 \\
Threat Predictability x SUD & .00 & $(1,112)$ & .99 & .00 \\
Overall Threat x SUD & .00 & $(1,112)$ & .95 & .00 \\
Congruence x SUD & 1.5 & $(1,112)$ & .22 & .01 \\
Threat Predictability x Congruence & .01 & $(1,112)$ & .91 & .00 \\
Overall Threat x Congruence & .09 & $(1,112)$ & .76 & .00 \\
Threat Predictability x Congruence x SUD & .04 & $(1,112)$ & .84 & .00 \\
Overall Threat x Congruence x SUD & 2.22 & $(1,112)$ & .14 & .02 \\
\hline
\end{tabular}

Note. ${ }^{*} p<.05,{ }^{* *} p<.01,{ }^{* * *} p<.001,{ }^{\wedge}$ marginal effect $(p>.05)$. 